\title{
ACTIVE AND PASSIVE FLOW CONTROL OVER THE FLIGHT DECK OF SMALL NAVAL VESSELS
}

\author{
Daniel M. Shafer \\ Thesis submitted to the Faculty of the \\ Virginia Polytechnic Institute and State University \\ in partial fulfillment of the requirements for the degree of
}

MASTER OF SCIENCE

in

Aerospace Engineering

APPROVED:

Dr. William H. Mason, chair

Dr. William Devenport

Dr. David B. Findlay

April 27, 2005

Blacksburg, Virginia

Keywords: helicopter/ship operations, frigate ship airwake, flow control, backward facing step 


\title{
ACTIVE AND PASSIVE FLOW CONTROL OVER THE FLIGHT DECK OF SMALL NAVAL VESSELS
}

\author{
Daniel M. Shafer
}

\section{ABSTRACT}

Helicopter operations in the vicinity of small naval surface vessels often require excessive pilot workload. Because of the unsteady flow field and large mean velocity gradients, the envelope for flight operations is limited. This experimental investigation uses a 1:144 scale model of the U.S. Navy destroyer DDG-81 to explore the problem. Both active and passive flow control techniques were used to improve the flow field in the helicopter's final decent onto the flight deck. Wind tunnel data was collected at a set of grid points over the ship's flight deck using a single component hotwire. Results show that the use of porous surfaces decreases the unsteadiness of the flow field. Further improvements are found by injecting air through these porous surfaces, causing a reduction in unsteadiness in the landing region of $6.6 \%$ at 0 degrees wind-over-deck (WOD) and $8.3 \%$ at 20 degrees WOD. Other passive configurations tested include fences placed around the hangar deck edges which move the unsteady shear layer away from the flight deck. Although these devices cause an increase in unsteadiness downstream of the edge of the fence when compared to the baseline, the reticulated foam fence caused an overall decrease in unsteadiness in the landing region of $12.1 \%$ at 20 degrees WOD. 


\section{DEDICATION}

This work is dedicated to my mother who passed away on January 31,2005 . She suffered from breast cancer for four years, yet always kept a positive outlook on life. She is an inspiration to all of us. She was loved greatly, is missed dearly, and will never be forgotten. I love you mom! 


\section{ACKNOWLEDGEMENTS}

This investigation would not have been possible without the support of the NATO AVT-102 task group. Great thanks goes to Terry Ghee for all the technical assistance, guidance, and mentoring he provided during this academic endeavor. Colin Wilkinson also provided guidance, contributed to the literature search and graphics, and helped me keep in contact with everyone upon completion of the testing phase. Robin Imber originally thought of the idea of testing the reticulated foam fence. Thanks to everyone in the Advanced Aerodynamics branch at Naval Air Systems Command, Patuxent River, MD for providing a friendly work environment, as I am happy to have had the opportunity to provide a contribution to their work. Thanks also goes to Dr. Bill Mason, my advisor, and Dr. Dave Findlay, head of the NAVAIR branch above. Without the cooperation of these two people, this project could not have happened. Finally, the entire faculty, staff, and Virginia Tech community have provided a graduate school experience that I would not trade for anything.

This work would also not have been possible without the personal support from my family and friends. My mother and father have provided more love and encouragement over the years than seems possible. I can only hope to be as good of a parent as they are. I have also always looked up to my big sister, Angela. Thank you all for being good role models. Finally, I must thank Theresa for putting up with me during all the stressful times in grad school. She made the experience much more enjoyable. 


\section{TABLE OF CONTENTS}

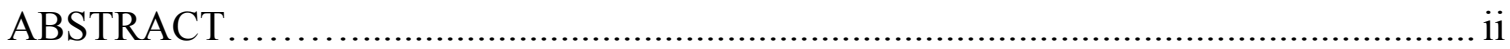

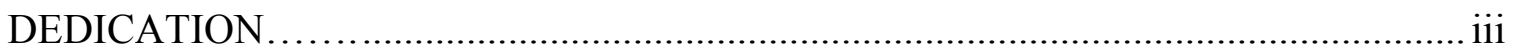

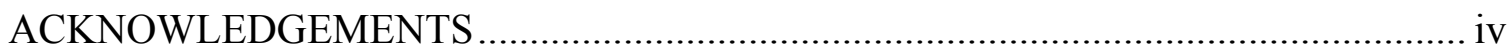

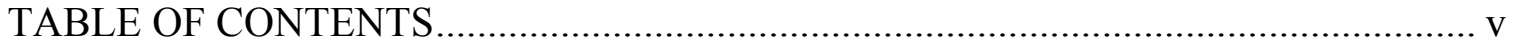

LIST OF FIGURES ...................................................................................... vii

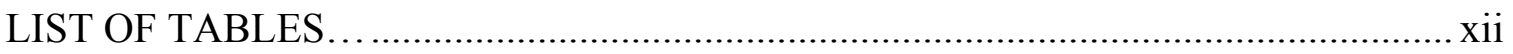

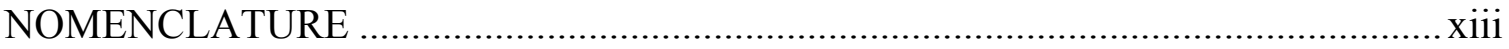

1. INTRODUCTION AND BACKGROUND …...................................................... 1

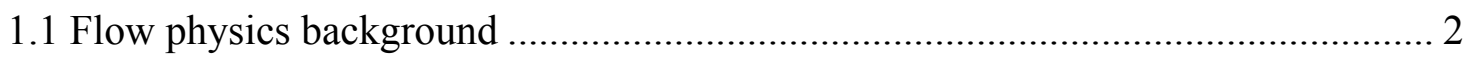

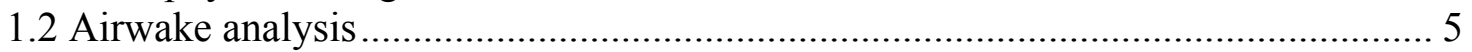

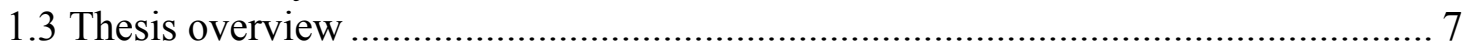

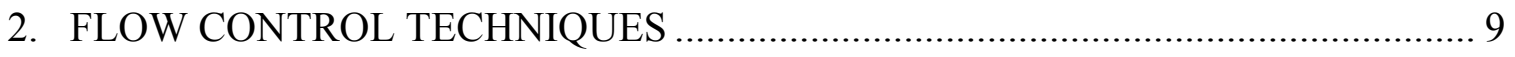

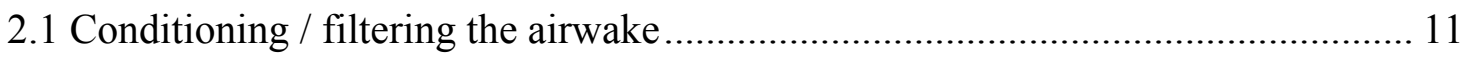

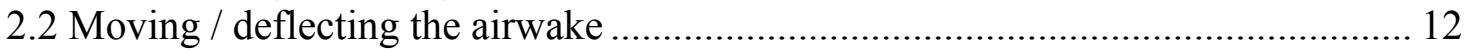

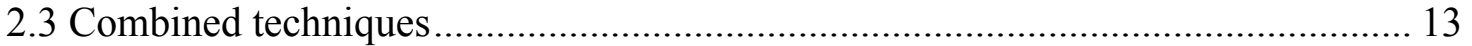

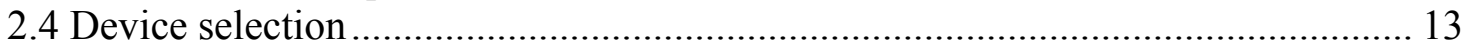

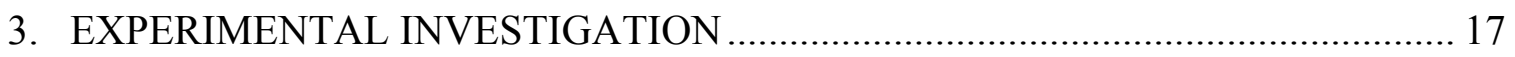

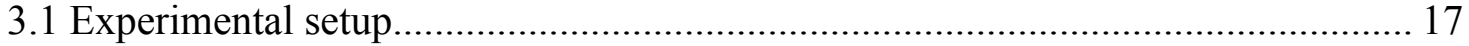

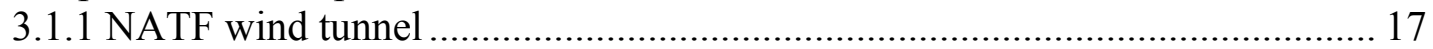

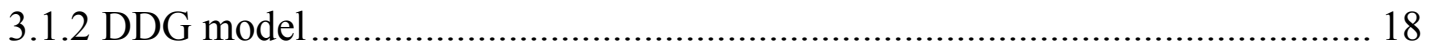

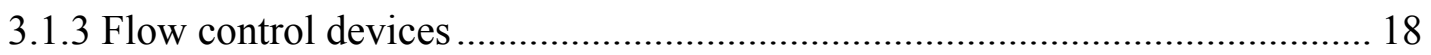

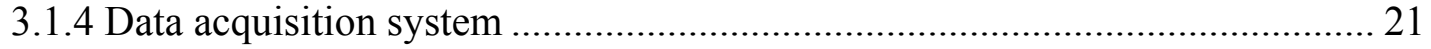

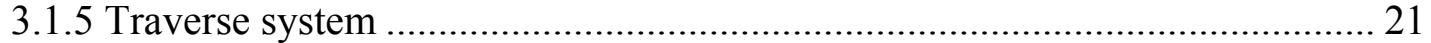

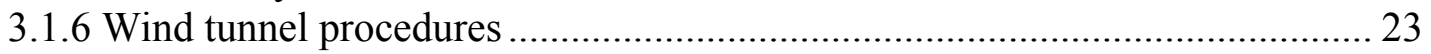

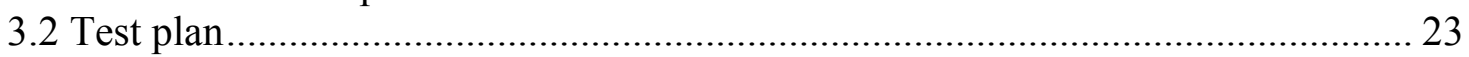

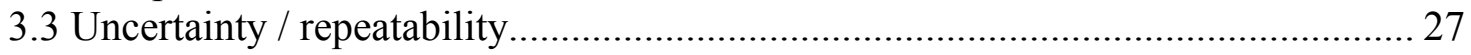

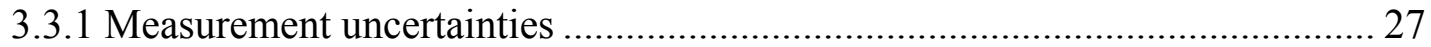

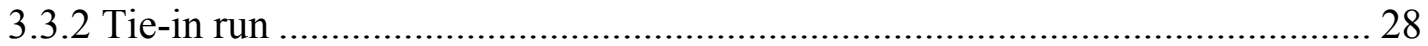

3.3.3 Measurement system random uncertainty .......................................... 28

4. RESULTS AND DISCUSSION: 0 DEGREES WIND-OVER-DECK..................... 33

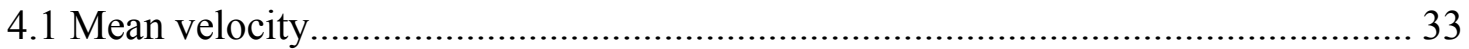

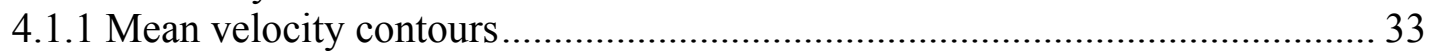

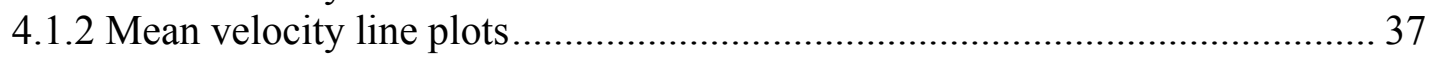

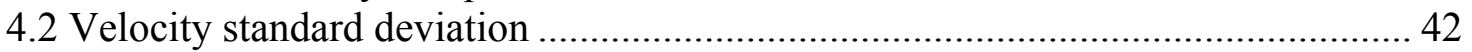


4.2.1 Velocity standard deviation contours........................................................ 42

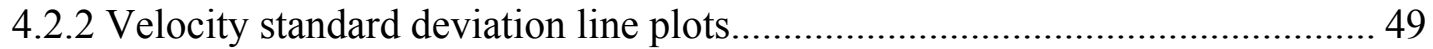

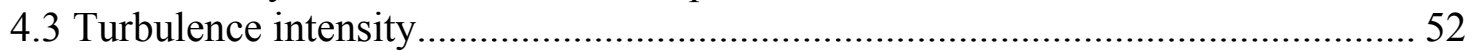

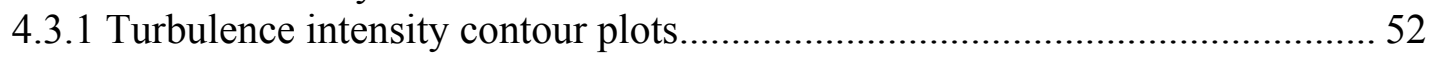

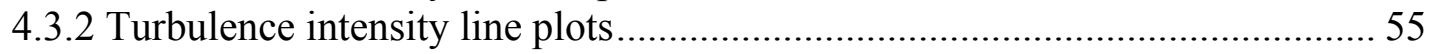

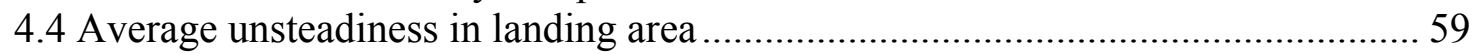

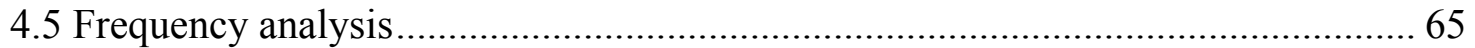

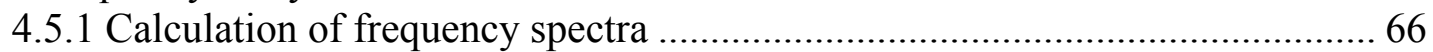

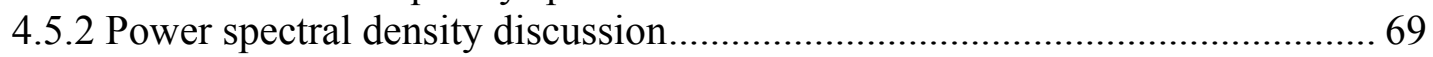

4.5.3 Frequency-separated standard deviation contours ..................................... 72

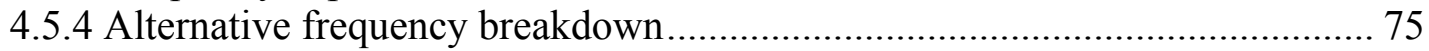

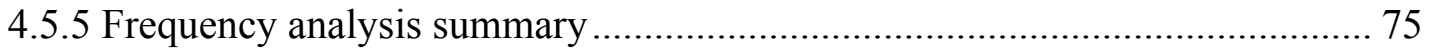

5. RESULTS AND DISCUSSION: 20 DEGREES WIND-OVER-DECK................... 77

5.1 Mean velocity................................................................................................. 78

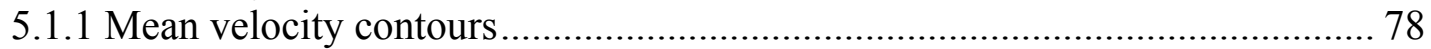

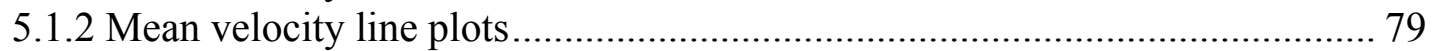

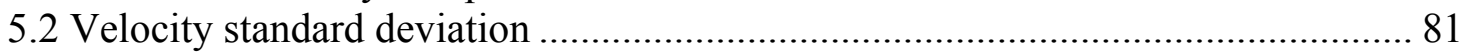

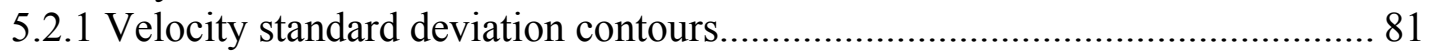

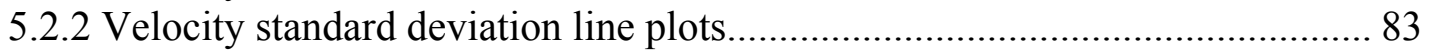

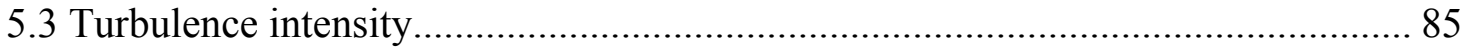

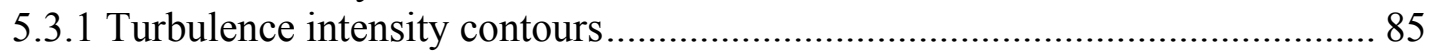

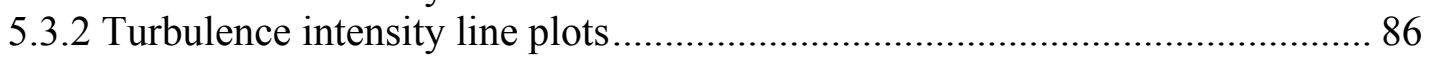

5.4 Average unsteadiness in landing area .......................................................... 88

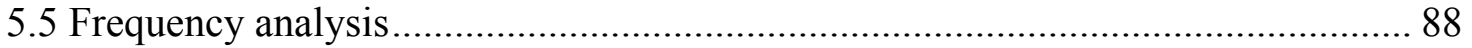

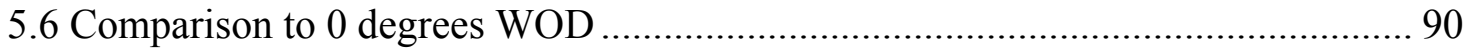

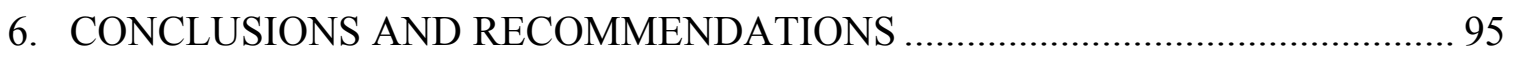

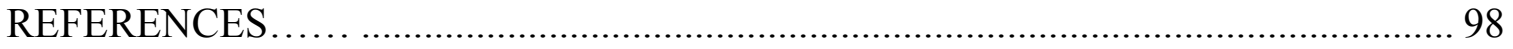

APPENDIX A: CALCULATION OF STANDARD DEVIATION ............................ 101

APPENDIX B: DERIVATION OF RESULTANT MEAN VELOCITY FOR SINGLE COMPONENT HOT WIRE ............................................................ 103

APPENDIX C: CONTOUR INTERPOLATION TECHNIQUE ............................... 105

APPENDIX D: POWER SPECTRAL DENSITY CALCULATION ......................... 108

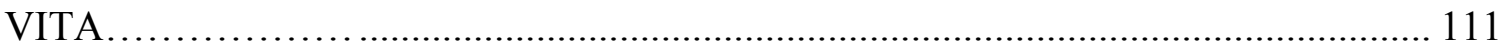




\section{LIST OF FIGURES}

Figure 1.1. Idealized flow over a backward facing step (Driver et al. ${ }^{7}$ )......................... 2

Figure 1.2. Simplified sketch of a ship's rear landing deck and fluid structure. .............. 4

Figure 2.1. Venn diagram of the categorization of flow control devices. ....................... 9

Figure 2.2. Sketches showing how each flow control device can be used.................... 10

Figure 2.3. Side view of the rear portion of the model showing the raised flight deck... 14

Figure 2.4. Fence flow control configurations under investigation............................ 15

Figure 3.1. NAVAIR Aerodynamic Test Facility ................................................... 17

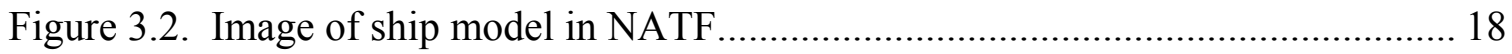

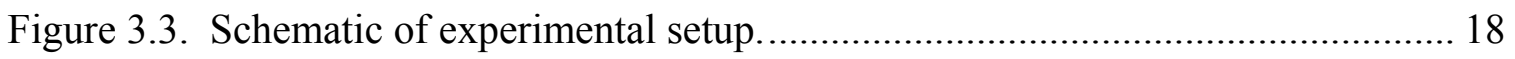

Figure 3.4. Construction of the raised flight deck and hangar extension. Note that the landing surface porous plate has not been installed yet..................................... 19

Figure 3.5. Float-type flow meter used to measure mass flow................................... 19

Figure 3.6. Side view of how injection is distributed in the cavities. The circular baffle (a) allows pressurization throughout the entire cavity. The diagonal baffle (b) channels all air to the hangar face......

Figure 3.7. Experimental data grid at 0 degrees yaw (a), and 20 degrees yaw (b) as

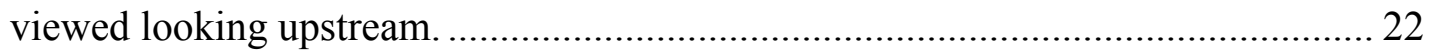

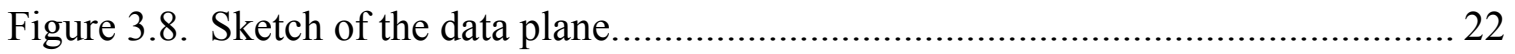

Figure 3.9. Differences between run 3 (left) and run 5 (right). ................................ 24

Figure 3.10. Velocity standard deviation above the center landing spot for a baseline model during (a) prior experiments and (b) current experiments......................... 28

Figure 3.11. Grid locations used to document the measurement system's random error.29

Figure 3.12. Velocity running average of independent time histories at a single spatial

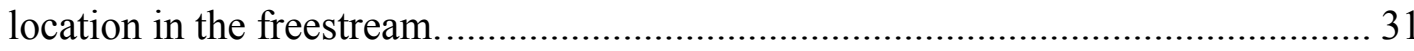

Figure 3.13. Velocity running average of independent time histories measured on the centerline at $z / h=0.34$.

Figure 3.14. Velocity running average of independent 32-second time histories measured on the centerline at $z / h=0.34$. 
Figure 4.1. Mean velocity of the baseline configuration (contours in \% freestream); (a), the entire flow field, and (b), the region of interest. .......................................... 34

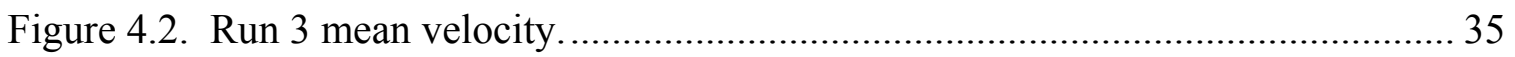

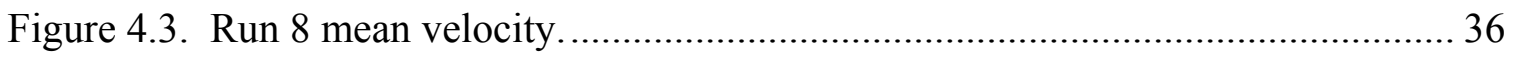

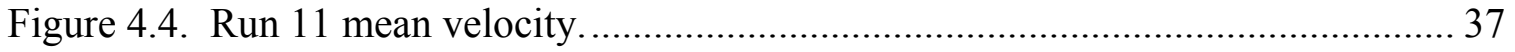

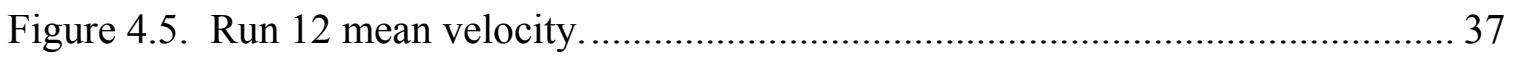

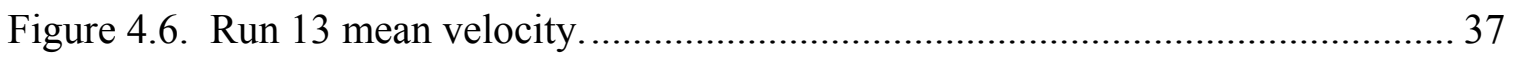

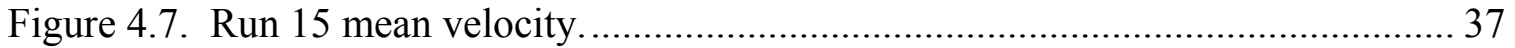

Figure 4.8. Mean velocity at low hover $(z / h=0.66)$ for porous surface configurations .39

Figure 4.9. Mean velocity at low hover $(z / h=0.66)$ for fence configurations............... 39

Figure 4.10. Mean velocity on the vertical centerline $(2 y / b=0)$ for porous surface configurations. 40

Figure 4.11. Mean velocity on the vertical centerline $(2 y / b=0)$ for fence configurations.

Figure 4.12. Velocity standard deviation of the baseline data; (a), the entire flow field, and (b), the region of interest.

Figure 4.13. Velocity standard deviation of vented porous surface configuration $(0.0625$ " holes, run 3). 44

Figure 4.14. Velocity standard deviation of non-vented porous surface configuration (0.0625" holes, run 5).

Figure 4.15. Velocity standard deviation of non-vented porous surface configuration (0.024" holes, run 6).

Figure 4.16. Velocity standard deviation of run 7 (injection using circular baffle)....... 46

Figure 4.17. Velocity standard deviation of run 8 (injection from hangar face)............46

Figure 4.18. Velocity standard deviation of run 9. 46

Figure 4.19. Velocity standard deviation of the serrated porous fence configuration (run 11). 47

Figure 4.20. Velocity standard deviation of the triangular-notched porous fence configuration (run12).

Figure 4.21. Velocity standard deviation of porous "mini delta wing" configuration (run 13). 
Figure 4.22. Velocity standard deviation of solid "mini delta wing" configuration (run 14).

Figure 4.23. Velocity standard deviation of reticulated foam fence configuration (run

$15)$. 48

Figure 4.24. Velocity standard deviation at low hover for porous surface configurations. 50

Figure 4.25. Velocity standard deviation at low hover for fence configurations. ........... 50

Figure 4.26. Velocity standard deviation along vertical centerline for porous surface configurations.

Figure 4.27. Velocity standard deviation along vertical centerline for fence configurations. 51

Figure 4.28. Turbulence intensity of the baseline configuration. 53

Figure 4.29. Turbulence intensity of run 3. 54

Figure 4.30. Turbulence intensity of run 8 . 54

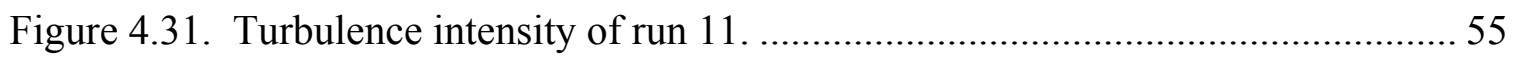

Figure 4.32. Turbulence intensity of run 12 ............................................................. 55

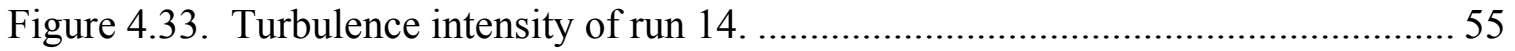

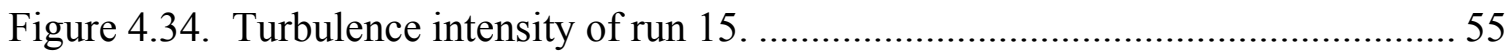

Figure 4.35 . Turbulence intensity at low hover $(z / h=0.66)$ for porous surface configurations. 57

Figure 4.36. Turbulence intensity at low hover $(z / h=0.66)$ for fence configurations.... 57 Figure 4.37. Turbulence intensity on the vertical centerline $(2 y / b=0)$ for porous surface configurations. 58

Figure 4.38. Turbulence intensity on the vertical centerline $(2 y / b=0)$ for fence configurations. 58

Figure 4.39. Baseline velocity standard deviation. The box encloses the region in the airwake which affects helicopter operations.

Figure 4.40. Percent change of the average unsteadiness in the landing region with respect to the baseline.

Figure 4.41. Width changes to the landing region $(|2 y / b|=1,1.4,1.7)$ overlaid on the velocity standard deviation contour plot. 
Figure 4.42. Height changes to the landing region $(z / h=1.9,2.4,2.9)$ overlaid on the

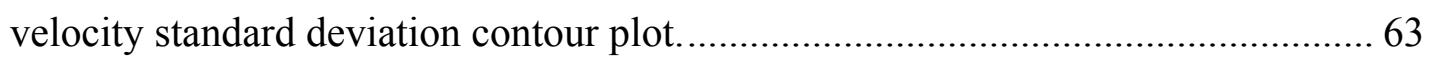

Figure 4.43. Bar graph showing the effect of widening the landing area........................ 64

Figure 4.44. Bar graph showing the effect of raising the height of landing area............. 65

Figure 4.45. Diagram of how data was divided to produce the PSD............................... 67

Figure 4.46. Effect of averaging several individual PSDs............................................ 68

Figure 4.47. Effect of passing the PSD through a low-pass digital filter (smoothed).... 68

Figure 4.48. PSDs of selected configurations at: a) $z / h=0.66$ and $2 y / b=0$; ) $z / h=0.66$

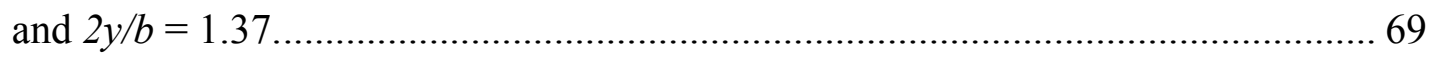

Figure 4.49. Velocity spectra following Kolmogorov's hypothesis............................... 71

Figure 4.50. Ratio of the standard deviation due to low frequencies to the total standard

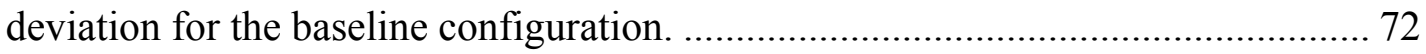

Figure 4.51. Baseline velocity standard deviation for: (a) the $0-125 \mathrm{~Hz}$ domain; (b) the

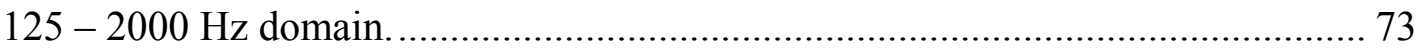

Figure 4.52. Velocity standard deviation for the porous flight deck with blowing from the hangar face for: (a) the $0-125 \mathrm{~Hz}$ domain; (b) the $125-2000 \mathrm{~Hz}$ domain..... 74

Figure 4.53. Velocity standard deviation for the porous serrated fence configuration for:

(a) the $0-125 \mathrm{~Hz}$ domain; (b) the $125-2000 \mathrm{~Hz}$ domain. 74

Figure 5.1. Mean velocity for the 20 degrees WOD baseline configuration. The contours are in percent of freestream velocity ..................................................................... 78

Figure 5.2. Mean velocity of reticulated foam fence configuration (run 17), ................ 79

Figure 5.3. Mean velocity of porous surfaces with injection configuration (run 18)..... 79

Figure 5.4. Mean velocity at low hover $(z / h=0.66)$ for 20 degrees WOD case............. 80

Figure 5.5. Mean velocity near the vertical centerline $(2 y / b=0.23)$ for 20 degrees WOD case.

Figure 5.6. Velocity standard deviation for the 20 degrees WOD baseline configuration.

The contours are in percent of freestream velocity. 82

Figure 5.7. Velocity standard deviation of reticulated foam fence configuration 82

Figure 5.8: Velocity standard deviation of porous surfaces with injection configuration 82 Figure 5.9. Velocity standard deviation at low hover $(z / h=0.66)$ for 20 degrees WOD case. 
Figure 5.10. Velocity standard deviation near the vertical centerline $(2 y / b=0.23)$ for 20

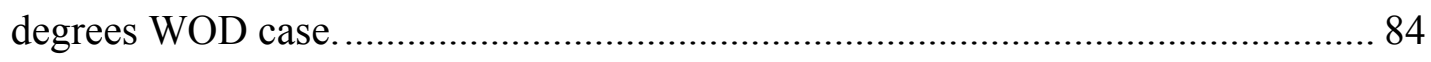

Figure 5.11. Turbulence intensity for the 20 degrees WOD baseline configuration....... 85

Figure 5.12. Turbulence intensity of the reticulated foam fence configuration (run 17). 86 Figure 5.13. Turbulence intensity of the porous surfaces with injection configuration (run $18)$. 86

Figure 5.14. Turbulence intensity at low hover $(\mathrm{z} / \mathrm{h}=0.66)$ for 20 degrees WOD case. 87 Figure 5.15. Turbulence intensity near the vertical centerline $(2 y / b=0.23)$ for 20 degrees WOD case. 87

Figure 5.16. Baseline velocity standard deviation for the 20 degrees WOD runs: 89

Figure 5.17. Velocity standard deviation at 20 degrees WOD for the porous flight deck with blowing from the hangar face for: a) the $0-125 \mathrm{~Hz}$ domain; b) the $125-2000$ $\mathrm{Hz}$ domain. 90

Figure 5.18. Velocity standard deviation at 20 degrees WOD for the reticulated foam fence configuration in: a) the $0-125 \mathrm{~Hz}$ domain; b) the $125-2000 \mathrm{~Hz}$ domain. . 90

Figure 5.19. Comparison of mean velocity at 0 and 20 degrees WOD at $z / h=0.66$..... 92 Figure 5.20. Comparison of standard deviation at 0 and 20 degrees WOD at $z / h=0.66$.

Figure 5.21. Comparison of mean velocity at 0 and 20 degrees WOD near the vertical centerline. 94

Figure 5.22. Comparison of standard deviation at 0 and 20 degrees WOD near the vertical centerline 


\section{LIST OF TABLES}

Table 2.1. Matrix of flow control devices................................................................ 10

Table 3.1. Run $\log$ for wind tunnel experiments. ......................................................... 26

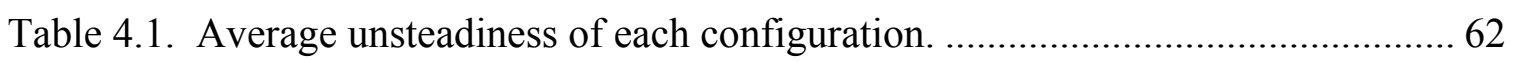




\section{NOMENCLATURE}

\section{Constants}

$\begin{array}{cll}\text { Symbol } & \frac{\text { Name }}{\text { Value }} \\ h & \text { Height of hangar } & 1.0 \mathrm{in} \\ b & \text { Beam of ship at the landing spot } & 4.375 \mathrm{in}\end{array}$

\section{Variables}

\begin{tabular}{|c|c|c|}
\hline Symbol & $\underline{\text { Name }}$ & $\underline{\text { Units }}$ \\
\hline$x$ & Streamwise coordinate & in \\
\hline$y$ & Lateral coordinate & in \\
\hline$z$ & Vertical coordinate & in \\
\hline$\dot{m}$ & Mass flow & $\mathrm{ft}^{3} / \min$ \\
\hline$U_{\infty}$ & Freestream velocity & $\mathrm{ft} / \mathrm{s}$ \\
\hline$u$ & Unsteady freestream velocity component & $\mathrm{ft} / \mathrm{s}$ \\
\hline$v$ & Unsteady lateral velocity component & $\mathrm{ft} / \mathrm{s}$ \\
\hline$w$ & Unsteady vertical velocity component & $\mathrm{ft} / \mathrm{s}$ \\
\hline$\sigma$ & Velocity standard deviation & $\mathrm{ft} / \mathrm{s}$ \\
\hline$\mu$ & Turbulent normal stress & $(\mathrm{ft} / \mathrm{s})^{2}$ \\
\hline TKE & Turbulent kinetic energy & $(\mathrm{ft} / \mathrm{s})^{2}$ \\
\hline $\mathrm{TI}$ & Turbulence intensity & - \\
\hline$\overline{U_{\text {local }}}$ & Local mean velocity & $\mathrm{ft} / \mathrm{s}$ \\
\hline WOD & Wind-over-deck angle & degrees \\
\hline
\end{tabular}




\section{INTRODUCTION AND BACKGROUND}

As long as helicopters have been flying, it has been desirable to land them aboard ships at sea. When these helicopter/ship operations began several decades ago, little was known about the unsteady airwake through which the helicopter must maneuver to land. Consequently, flight operations around small naval vessels have historically been one of the most difficult skills for a helicopter pilot to master. Even with years of experience, a pilot cannot completely anticipate how the unsteady airwake behind a ship will behave. Over the years numerous accidents have occurred during flight operations, causing lives to be lost and millions of dollars of damage. As a result, efforts to characterize and

predict the flow behavior due to the ship airwake began more than a decade ago. ${ }^{1,2}$ Although studies on the subject have become more common in recent years, little knowledge exists on ways to control and reduce the unsteady wake that helicopters encounter in this situation. Consequently, the existing knowledge of the physics of ship airwakes should be examined to find a solution which reduces airwake turbulence and allows for safer helicopter/ship operations over a wider range of flight conditions.

Most current small naval ships were designed without considerations for the airwake encountered during takeoff and landing maneuvers. Although airwake analysis is now an important part of new ship designs, an effort must also be made to increase the safety of personnel on those vessels currently in the fleet. This investigation focuses on finding techniques which improve the airwake over the rear landing deck of frigate-type ships, specifically the DDG class. 


\subsection{Flow physics background}

In its most basic form, flow over the rear landing deck aft of the hangar on frigate-type ships can be thought of as flow over a backward facing step. Experimental and computational literature is readily available for two dimensional versions of this flow. ${ }^{3,4,5,6,7,8,9}$ Figure 1.1 illustrates the general characteristics of this two dimensional flow. The dominant flow feature is the large recirculation region beneath the unsteady, separated shear layer. This shear layer acts like a mixing layer, as coherent structures are formed and transported downstream initially. However, the lives of these structures are very short since they breakup prior to reattachment to the surface. Because this process is chaotic, reattachment is not stationary and must be characterized by a region, not a point. This is one of the reasons why helicopter landings are difficult; the landing spot is near this reattachment region.

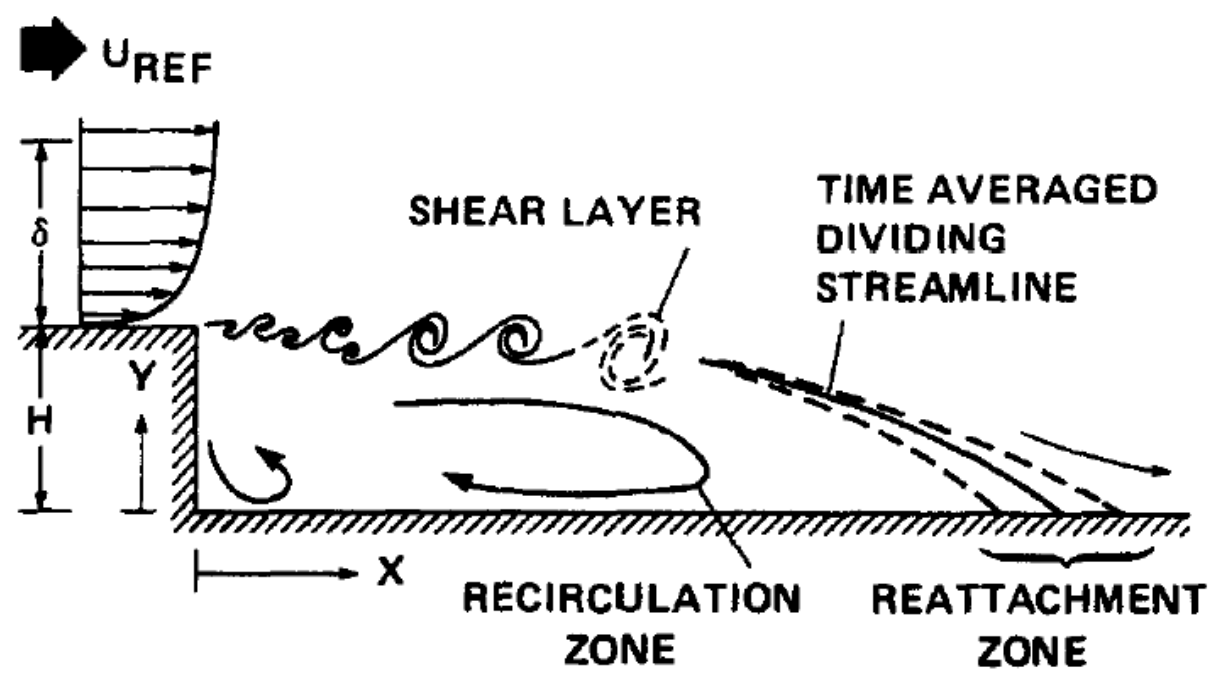

Figure 1.1. Idealized flow over a backward facing step (Driver et al. ${ }^{7}$ ). 
Although this fundamental understanding of two dimensional flow over a backward facing step is available, its applicability to flight deck flow fields is limited. There are two primary concerns. First, the flow over a ship is far from two dimensional, as the ratio of beam width to step height is approximately three. This aspect ratio is an important parameter in flows with massive separation regions, ${ }^{10}$ and the three dimensionality is important in this application. A second reason for the limited applicability involves the condition of the oncoming flow. Because of upstream bodies on the ship (stacks, antennas, mast, etc.), the flow over the step is not uniform. This complicates matters as the oncoming flow is completely unsteady and nearly impossible to quantify. As a result, analyzing simplified models of this type of flow may not be particularly useful.

Extending the backward facing step flow to three dimensions, consider flow perpendicular to the top hangar edge. Literature ${ }^{11,12,13}$ suggests the characteristics shown in Figure 1.2, discovered though extensive flow visualization. Similar to the two dimensional case, a large recirculation region exists behind the step; however, this flow field is no longer two dimensional. On the flight deck, flow is incoming from the sides of the ship causing counter-rotating vortices on each side of the recirculation region. This results in a horseshoe vortex structure. Keep in mind, however, that this structure is not stationary. As a result, the natural unsteadiness of the flow causes this structure to grow, dissipate, and move spatially in an unpredictable manner. 


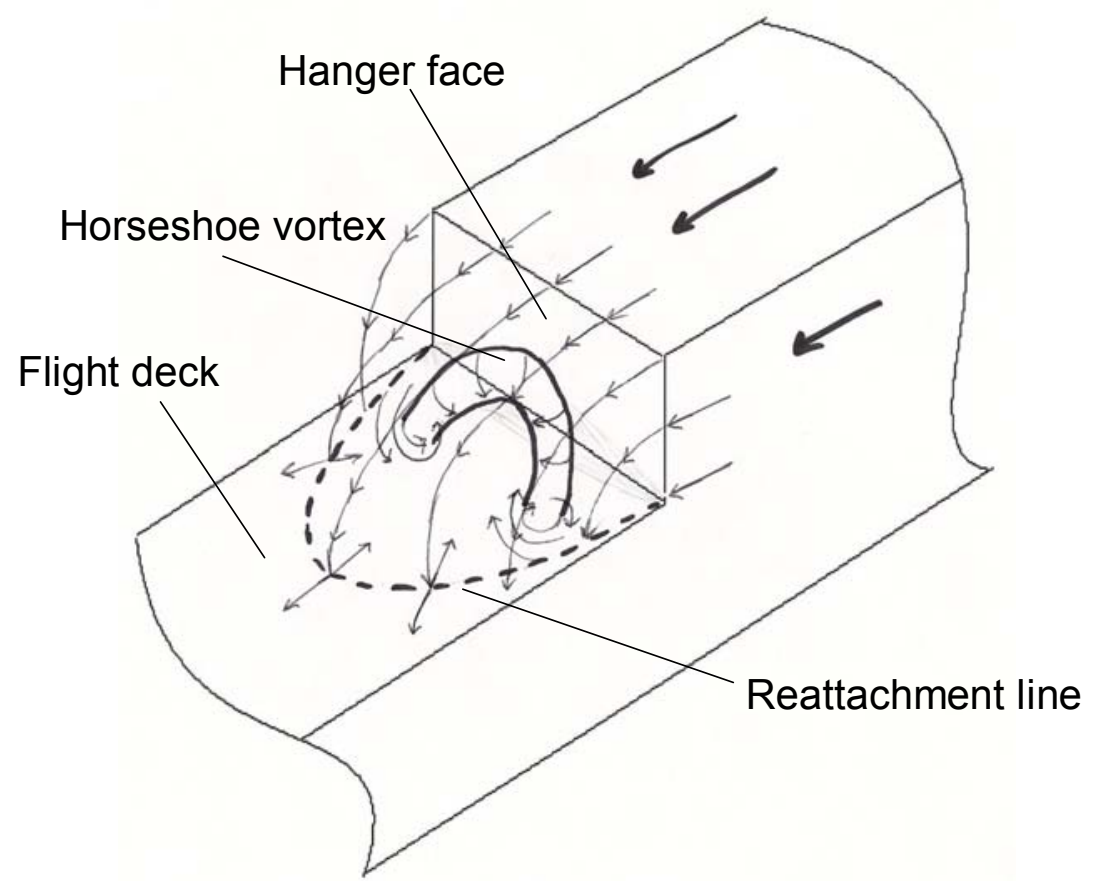

Figure 1.2. Simplified sketch of a ship's rear landing deck and fluid structure.

Adding another degree of complexity, one must also consider situations when the freestream has a crosswind component. These nonzero wind-over-deck (WOD) angles (or yaw angles) occur quite frequently, as it is rare for a ship to be cruising on a heading such that the WOD is directly down the centerline. As a result, these situations require study. The literature ${ }^{11,12,13}$ also characterizes the flow around frigate-type ships at several WOD angles. Again, various types of flow visualization were used to define a flow model in the vicinity of the landing deck on the ship. Rhoades ${ }^{11}$ showed that at moderate WOD angles (approximately 45 degrees), large coherent structures are shed from the side edge of the hangar at regular intervals. Unfortunately, documentation of this phenomenon on full scale ships is not available. However, this type of predictable periodicity may not even occur at full scale for several reasons. First, models in a wind 
tunnel are typically scaled down in size substantially. As with any bluff body, these flows are Reynolds number and Strouhal number dependent, and the large scaling factors are of concern. Additionally, the preexisting atmospheric boundary layer and turbulence are infrequently modeled. It has been shown that accounting for these features is critical when WOD angles approach 90 degrees. ${ }^{14}$

In summary, the simplified two and three dimensional backward facing step flows provide a good physical overview of the flow encountered over the flight deck of frigatetype ships. Recall that helicopter operations are difficult because the entire recirculation region is unsteady and the landing spot on the flight deck is near the shear layer reattachment region. Because of the flow field's unsteadiness, a helicopter could be inside the recirculation region at one instant, experiencing certain forces and moments, only to be subjected to an unrecoverable yawing moment outside the recirculation region at the next instant. Thus, to reduce pilot workload, it is desirable to decrease flow gradients and remove as much of the flow field unsteadiness as possible over the flight deck.

\subsection{Airwake analysis}

Recently, with the development of more advanced CFD methods and increased computing power, numerical simulations and capabilities have dramatically improved. Results from CFD solutions for ship airwakes are available in the literature. ${ }^{15,16,17,18}$ Solutions often use time-accurate calculations and agree with wind tunnel and flight test

data fairly well. In one case ${ }^{19}$ the CFD solution is used with a flight simulator enabling a pilot to fly through the airwake. This gives the pilot the ability to determine how realistic 
the solution model is. Thus, CFD has shown some success and will continue to improve as the technology advances in the future. These simulations can accomplish two main tasks for a fraction of the cost of full scale flight test. First, pilots are able to train in simulators in a wide variety of unique ship airwakes. Allowing training in a simulator will better prepare pilots for actual landings aboard ships and decrease the amount of actual flight time needed to develop these skills. It is also anticipated that manned flight simulations will be used to define the helicopter/ship operating envelope. Every time an aircraft or ship design is modified, the operating envelope must be redefined. Currently the only way to do this is by actual flight test, and although simulations are not intended to be a replacement, the number of test points can be greatly reduced using simulations. This will result in significant time and cost savings.

Wind tunnel experiments can also aide in airwake analysis. In addition to the flow physics experiments mentioned above, other researchers have mapped the flight paths of the aircraft approach to different ships. ${ }^{20,21}$ This wind tunnel data was used not only for a better understanding of the flow field, but also for CFD validation and an airwake model in a flight simulator. Other wind tunnel experiments ${ }^{22}$ measuring unsteady forces and moments on a helicopter fuselage show promise in predicting the operating limits for helicopter operations. By correlating the magnitudes of these unsteady fluctuations to pilot workloads from full scale flight test, operating envelopes were approximated. Again, by minimizing the amount of flight test, time and money can be saved. It has also been shown that rotor downwash has a significant effect on the flow field, ${ }^{23}$ and the flow field over the landing deck causes a decrease in rotor thrust. ${ }^{24}$ Thus, to get the most realistic results from ship airwake experiments, one must model the 
helicopter fuselage, including the rotor. Finally, ship motion can also affect the airwake. The unsteady six degree-of-freedom movements of the ship result in much greater flow field complexities, and little documentation of this effect is available. ${ }^{25}$

Because the helicopter/ship interface is such a complex problem and much effort has been placed on attempting to understand the phenomenon, efforts to actually "fix" the problem have been insufficient. Although a large amount of information is available for backward facing step flows, these works are rarely applied to small ship flow fields. However, it was shown that porous fences can be used to significantly reduce unsteadiness and decrease the mean velocity downstream of the step in two related studies. ${ }^{26,27}$ Though the studies' motivation came from the helicopter/ship dynamic interface problem, only two dimensional configurations were explored. Consequently, much work and effort needs to be conducted in this flow control application. Presently, an increased interest from around the world has resulted in support from NATO to explore the problem in greater detail. With the existing knowledge of the physics of ship airwakes, it is hoped that flow control techniques can be designed to increase the safety and expand the operating limits of helicopter/ship operations. In conclusion, this investigation is just the first step in developing these flow control techniques. Many of the secondary effects (atmospheric boundary layer, rotor downwash, ship motion, etc.) mentioned above are not considered.

\subsection{Thesis overview}

The next chapter introduces the many different techniques that can be used to modify and reduce the flow field nonuniformity and unsteadiness over the flight deck of 
frigate-type ships. This leads to a discussion of the specific techniques chosen for this investigation. Chapter 3 explains the experimental setup, procedures, and the instrumentation used to collect data. A composite test plan and run log is presented along with an in depth uncertainty analysis of the entire hotwire measurement system. Chapter 4 contains the experimental results for configurations at 0 degrees wind-over-deck (WOD). The majority of the investigation was spent evaluating flow control techniques at this WOD angle. Several different types of analysis are discussed as there is no specific metric widely accepted for evaluation of flow control devices at this time. In Chapter 5, the two best control techniques from the 0 degree WOD cases are tested at 20 degrees WOD. A comparison between these two types of flow is also included. Finally, the summary and conclusions are found in Chapter 6. Several recommendations are made for future studies. 


\section{FLOW CONTROL TECHNIQUES}

Numerous flow control techniques have been considered and fall into two broad categories. First, the flow can be conditioned or filtered in some way as it passes over the backward facing step and landing deck. The goal is to remove some of the energy in the flow or possibly shift the magnitude of the unsteadiness in the frequency domain. The other category involves devices which physically move or deflect the shear layer. The motivation for this concept is to move highly turbulent flow out of the region in which helicopters operate. Figure 2.1 is a diagram of how several novel devices fit into these two categories. Note that some devices fit into both categories. Table 2.1 is a tabulated version which includes references to sketches of the devices shown in Figure 2.2.

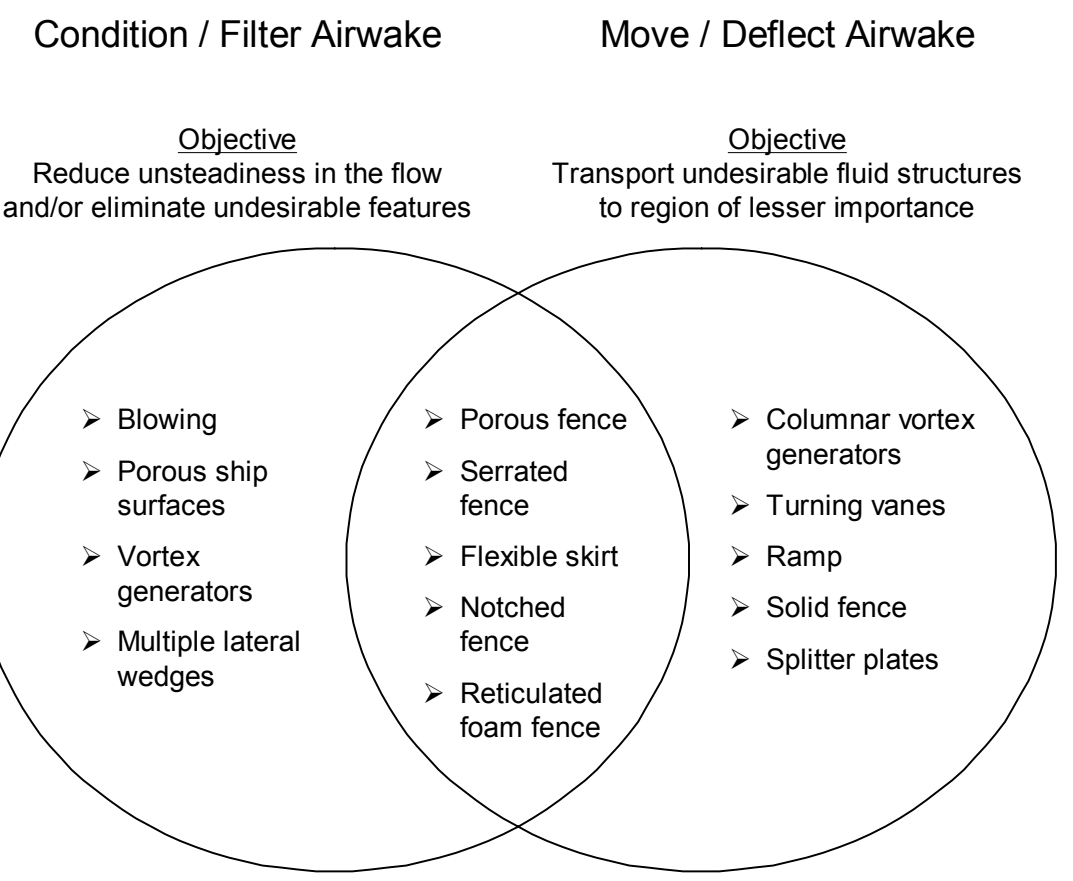

Figure 2.1. Venn diagram of the categorization of flow control devices. 
Table 2.1. Matrix of flow control devices

\begin{tabular}{|c|c|c|}
\hline Category & Desired effect & Control device / technique \\
\hline \multirow{4}{*}{$\begin{array}{c}\text { Condition / filter } \\
\text { airwake }\end{array}$} & \multirow{4}{*}{$\begin{array}{l}\text { Reduce unsteadiness } \\
\text { in the flow and/or } \\
\text { eliminate undesirable } \\
\text { features }\end{array}$} & 1. Blowing \\
\hline & & 2. Porous ship surfaces \\
\hline & & 3. Vortex generators \\
\hline & & 4. Multiple lateral wedges \\
\hline \multirow{5}{*}{\multicolumn{2}{|c|}{ Both }} & 5. Porous fence \\
\hline & & 6. Serrated fence \\
\hline & & 7. Flexible skirt \\
\hline & & 8. Notched fence \\
\hline & & 9. Reticulated foam fence \\
\hline \multirow{5}{*}{$\begin{array}{l}\text { Move / deflect } \\
\text { airwake }\end{array}$} & \multirow{5}{*}{$\begin{array}{l}\text { Transport undesirable } \\
\text { fluid structures to region } \\
\text { of lesser importance }\end{array}$} & $\begin{array}{l}\text { 10. Columnar vortex } \\
\text { generators }\end{array}$ \\
\hline & & 11. Turning vanes \\
\hline & & 12. Ramp \\
\hline & & 13. Solid fence \\
\hline & & 14. Splitter plates \\
\hline
\end{tabular}

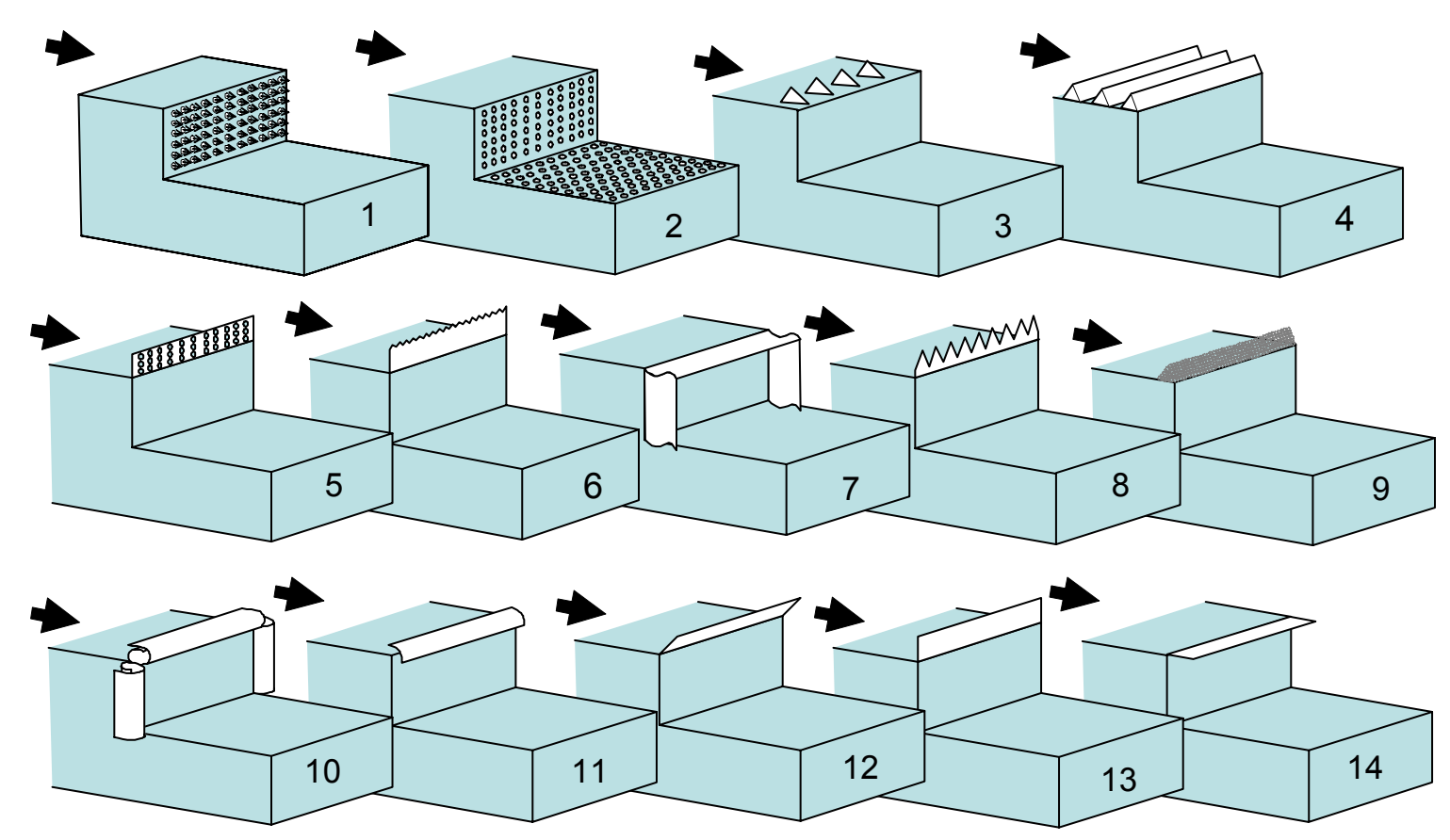

Figure 2.2. Sketches showing how each flow control device can be used. 


\subsection{Conditioning / filtering the airwake}

Ideally, all the devices in Figure 2.2 require test and evaluation; however, some are believed to perform better than others by simply considering the physics of the flow. First, the condition/filter category is considered. Literature suggests that a porous surface downstream of a backward facing step can decrease the unsteadiness in the recirculation region and lower the amount of reversed flow. ${ }^{28,29}$ Porous surfaces allow the passage of local high pressures to areas of low pressure, thus decreasing the magnitude of the fluctuations on the surface. In addition to reducing the peak RMS pressure fluctuations, particle image velocimetry (PIV) vector fields show shrinkage of the recirculation region. While these were two-dimensional studies, the concept of "damping" the flow field near the surface of the flight deck is applicable here. To further improve the flow field with this configuration, the cavity beneath the porous surface could also be pressurized, resulting in mass injection into the freestream. Because of the natural low pressure region below the step, ${ }^{13}$ providing a positive mass flow here helps alleviate pressure gradients. Furthermore, if the injection were in the direction of the freestream (out of the vertical face of the step as shown in configuration 1 in Figure 2.2), energy would be added in the direction of the velocity deficit.

The remaining two devices in this category could actually increase the unsteadiness. Although vortex generators are commonly used to keep flow attached and prevent wakes from developing, they are primarily used on streamlined objects which require energy be added to the boundary layer. In this application, the upstream flow is already chaotic due to the airwake of upstream bluff bodies (the ship's mast, stacks, antennas, etc.). Although vortex generators could possibly improve the flow by breaking 
up large eddies and shifting the turbulent kinetic energy to a higher frequency domain, this method was not pursued.

Another method to condition the airwake, not included in the sketches, is to use active unsteady devices. Differing from constant uniform injection, these techniques have a time dependent effect on the flow field. Perhaps the rotation speed of a wind turbine is varied or a pulsed jet is used to decrease the magnitude of the unsteady fluctuations. Creative techniques like these represent a completely new class of flow control devices yet to be explored in this application.

\subsection{Moving / deflecting the airwake}

Now consider the move/deflect category. These techniques attempt to physically move the unsteady shear layer in two basic ways. Solid fences, ramps, and columnar vortex generators $(\mathrm{CVGs})^{30}$ move the shear layer away from the region of helicopter operations. Although these devices can decrease the unsteadiness in some regions, the intensity of the shear layer or unsteadiness is often greatly magnified in other regions, specifically downstream of the fence edge. Turning vanes and splitter plates attempt to

move the shear layer down towards the flight deck. However, other current research ${ }^{31}$ shows that these techniques may be completely nullified by the interaction of rotor downwash. This dynamic interface has effects on all devices, but may be more significant on devices which attempt to move the flow vertically. Experimentally determining this interaction is difficult due to the need of a high fidelity, fully functioning rotor model to place into the flow field. 


\subsection{Combined techniques}

Thus far, all the devices and techniques discussed have fallen solely into either one category or the other. Most of these devices have advantages and disadvantages. As a result, it seems natural to attempt to combine the strategies of both categories. This leads to the devices cited in the middle of Figure 2.1 and Table 2.1. This blended category consists mainly of fences which are either non-rectangular or porous. By shaping a solid rectangular fence to promote mixing, the severity of the shear layer initiated at the top of the fence is reduced. In other words, if the fence is not rectangular, the shear layer will be broken up as it is deflected. In addition, the flow could also be "filtered" by using porous materials. This idea is inspired by the use of screens and honeycomb in wind tunnels to reduce turbulence. Two materials which can be used are porous sheet metal and reticulated foam (configurations 5 and 9 respectively in Figure 2.2). Using these two philosophies - shaping and porosity - several unique fences were selected for testing.

\subsection{Device selection}

Because it is not possible to test every type of technique listed in Table 2.1, a few

of the most appealing were selected and explored in depth. Because multiple sources ${ }^{28,29}$ have shown flow improvements with the use of porous surfaces, this technique was explored in greater detail. To establish a better understanding of how porosity affects the flow field, several different configurations were tested using this technique. Upon the fabrication of such configurations, it is noted that the original DDG model is modified with a raised flight deck (see Figure 2.3). This was essential to allow a cavity beneath the 
porous surface. As a result, it was necessary to document the effect of "venting" the cavity. More specifically, the original sidewalls of the cavity were constructed using porous material, but data was also taken with solid cavity walls. Additionally, the effect of hole diameter was explored as the porosity of the surfaces was held constant at $23 \%$. After these passive techniques were explored, blowing was added to the system. Injection could be used in a variety of ways, but was used here to attempt to pressurize the cavities. Further details of individual configurations are discussed in Section 3.1.3.

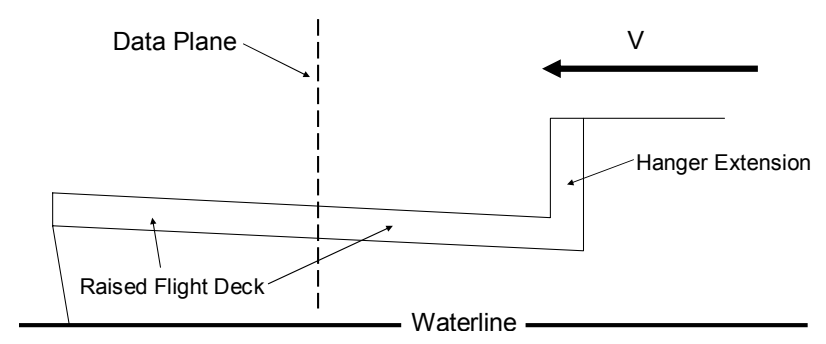

Figure 2.3. Side view of the rear portion of the model showing the raised flight deck.

Fence devices were also explored. For the reasons discussed previously, focus was placed on fences falling into both categories in Table 2.1. It has been shown that porous fences cause a less severe shear layer than a solid fence with the same dimensions. ${ }^{32}$ Referring to Figures $2.4 \mathrm{a}$ and $\mathrm{b}$, the serrated porous fence and triangularnotched porous fence attempt to break up the shear layer into smaller sized eddies and deflect the remaining shear layer away from the flight deck. Figures $2.4 \mathrm{c}$ and $\mathrm{d}$ show triangular-notched fences that are deflected 60 degrees upstream. The motivation for this configuration comes from the natural characteristics of delta wings at high angles of attack. It is hypothesized that tiny leading edge vortices develop and break up larger scale eddies, leaving much smaller, higher frequency structures in the flow over the deck. 
This is a desirable characteristic because low frequency unsteadiness is the main cause of handling quality problems for pilots. ${ }^{33}$ Finally, Figure $2.4 \mathrm{e}$ consists of a reticulated foam fence. The material is commercially available and is commonly used for padding and sound absorbing. A triangular cross-section was chosen in an attempt to blend the shear layer smoothly into the pre-existing airwake. In other words, the thickness of the fence decreases with height. Note that the fences were placed around the edges of the hangar, including the sides.

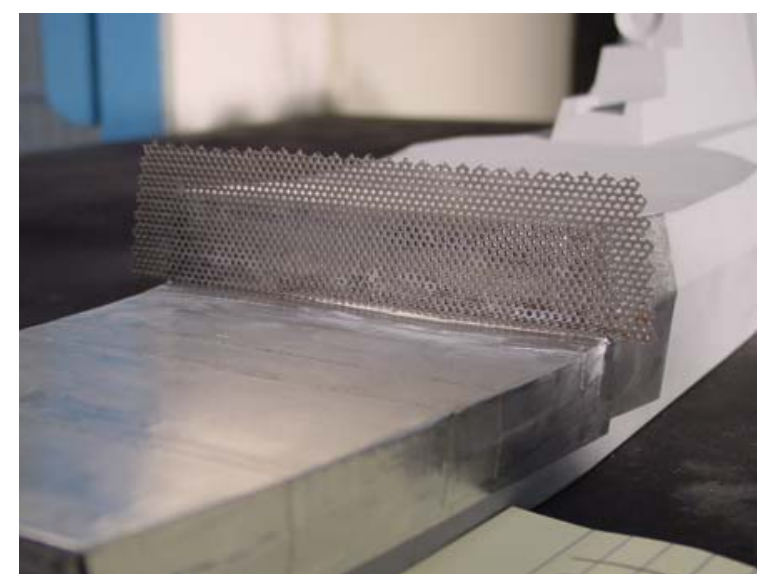

a. Serrated porous fence.

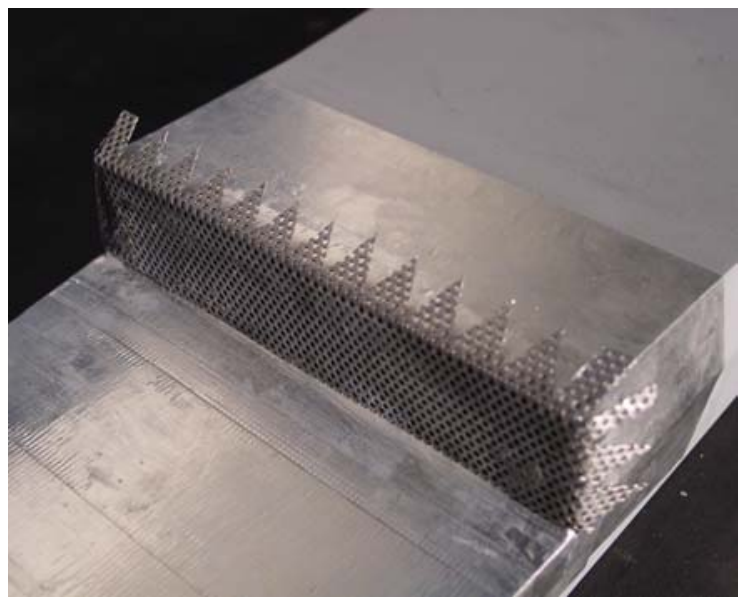

c. Triangular-notched porous fence, angled 60 degrees upstream.

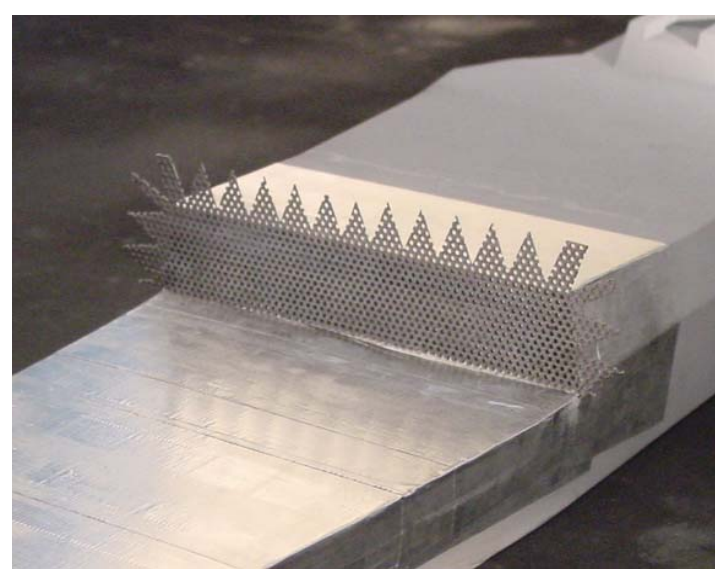

b. Triangular-notched porous fence.

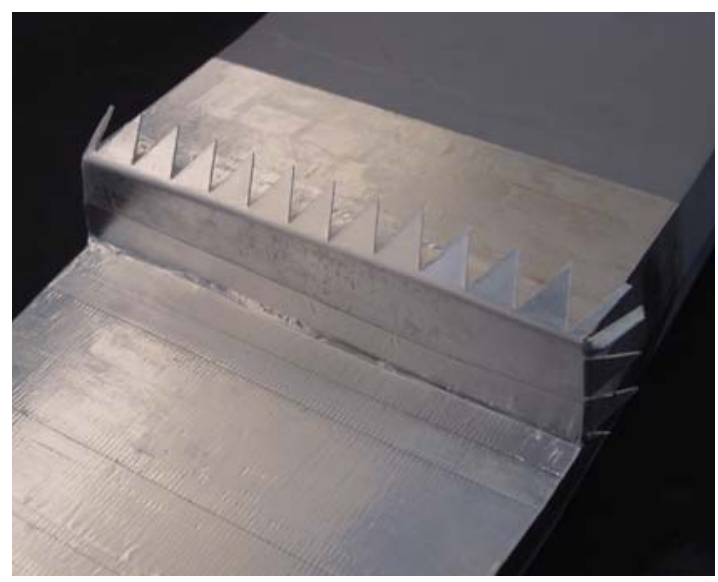

d. Triangular-notched solid fence, angled 60 degrees upstream.

Figure 2.4. Fence flow control configurations under investigation. 


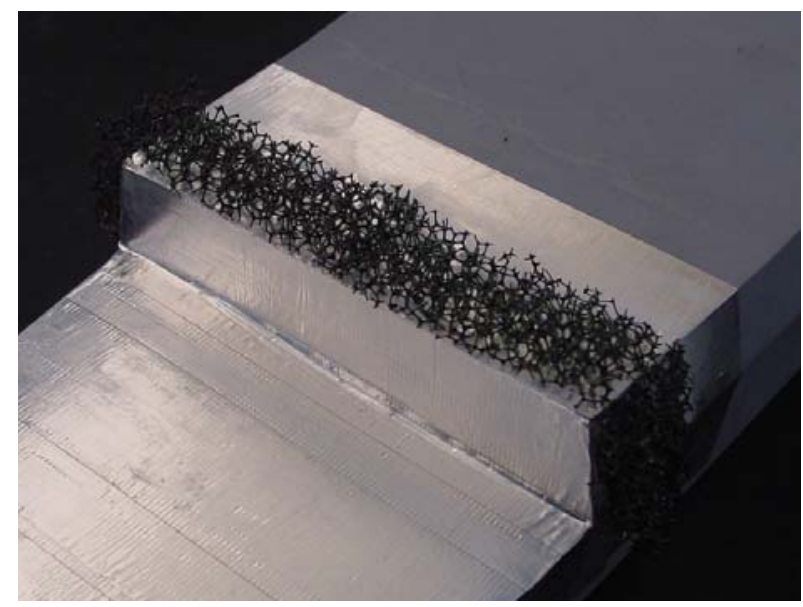

e. Reticulated foam fence.

Figure 2.4. Fence flow control configurations under investigation. 


\section{EXPERIMENTAL INVESTIGATION}

\subsection{Experimental setup}

\subsubsection{NATF wind tunnel}

The wind tunnel tests were conducted at the NAVAIR Aerodynamic Test Facility $(\mathrm{NATF})^{34}$ in Patuxent River, Maryland. The NATF is a 4-foot by 4-foot closed test section, open-return wind tunnel (see Figure 3.1). The facility incorporates a 200 horsepower motor that drives a variable pitch fan and delivers a maximum velocity of 205 feet per second. In addition, the wind tunnel has honeycomb and three sets of flow conditioning screens that minimize freestream turbulence intensity to approximately $0.80 \%$ and freestream velocity variations of $1 \%$ or less.

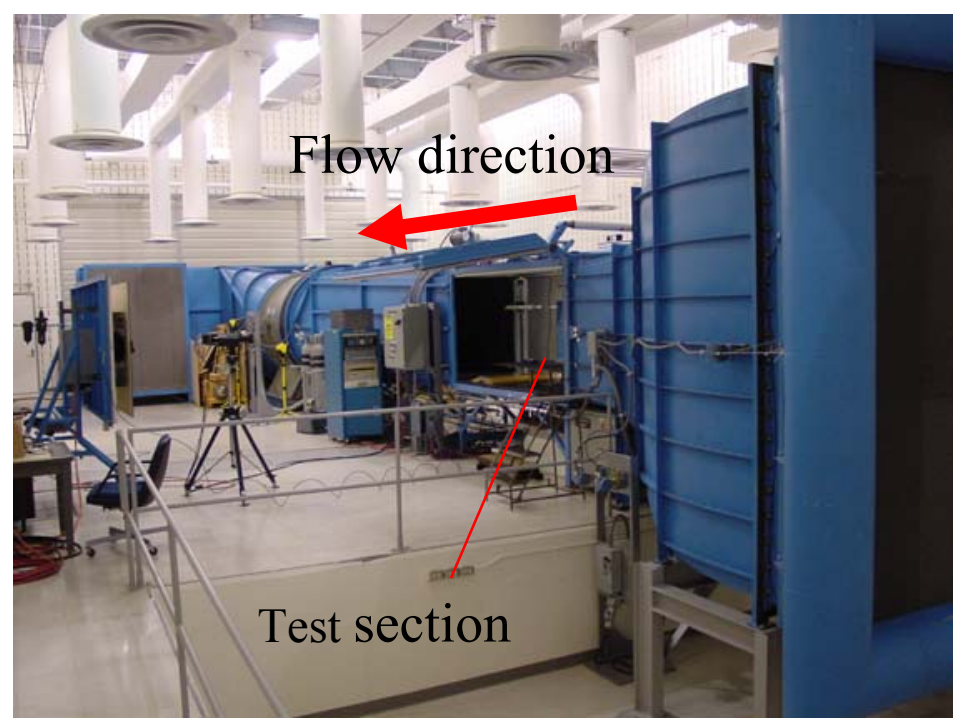

Figure 3.1. NAVAIR Aerodynamic Test Facility. 


\subsubsection{DDG model}

The model of the 1:144 scale DDG-81 ship, manufactured by NASA Langley Research Center using stereo lithography techniques, was approximately 42 inches in length. The beam of the ship on the rear flight deck varied from 4.9375 inches at the hangar face to 3.5625 inches at the back edge of the ship. The model was affixed to a boundary layer plate (Figure 3.2 and 3.3), 62 inches in length by 46.5 inches in width, and was mounted in the tunnel 8 inches above the test section floor. An aerodynamic profile was attached to the nose and trailing edge of the boundary layer plate. The trailing edge piece was mounted upside down relative to the nose to assist wake mitigation and recovery. A trip strip, composed of $1 / 8$ inch wide, 80 grit sand paper was glued to the leading edge of the boundary layer plate on the lower ( $7 / 8$ inch downstream from the leading edge) and upper surface (1/4 inch downstream from the leading edge).

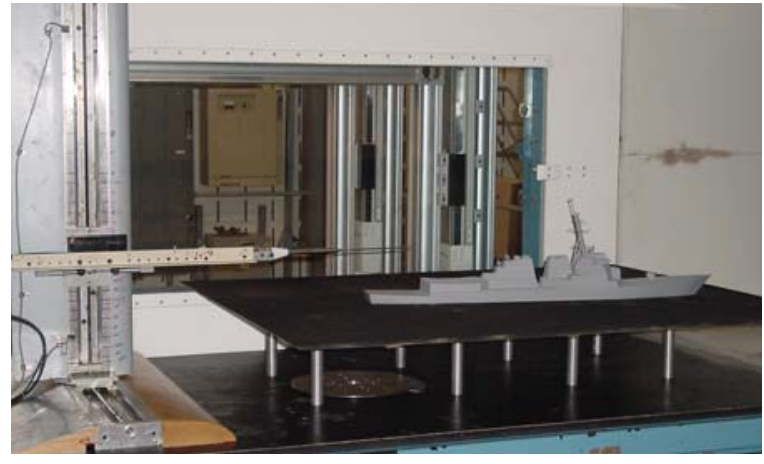

Figure 3.2. Image of ship model in NATF.

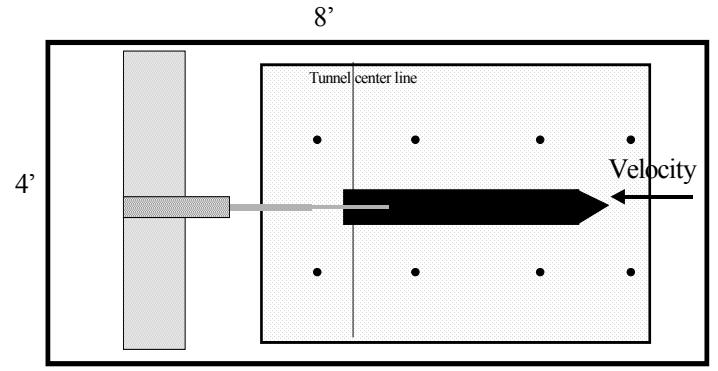

Figure 3.3. Schematic of experimental setup.

\subsubsection{Flow control devices}

Sheet metal with two different sizes of machined holes served as the porous surfaces. The raised flight deck and hangar extension were shaped to lay flush on the 
existing DDG model and were elevated by affixing the surface to small cubes (3/8 inch) as seen in Figure 3.4. The hangar face was constructed in a similar manner. Note that aluminum tape can be placed on any porous surface to simulate the solid surfaces discussed in the device selection section. Also note the hole in the original flight deck surface just aft of the hangar. This $1 / 2$ inch diameter hole extended through the model and ground plane so that an airline could be installed for active flow control configurations. The location on the landing deck for this airline port was on the centerline of the flight deck one inch aft of the hangar face, chosen for the location of minimum pressure. ${ }^{13} \mathrm{~A}$ $1 / 8$ inch diameter tube was then fitted to the bottom of the model. The tube exited the tunnel under the boundary layer plate and passed through a mass flow meter which used shop air as the pressure source. The regulator pressure was set nominally to 90 pounds per square inch, but airflow was controlled by the float-type flow meter shown in Figure 3.5.

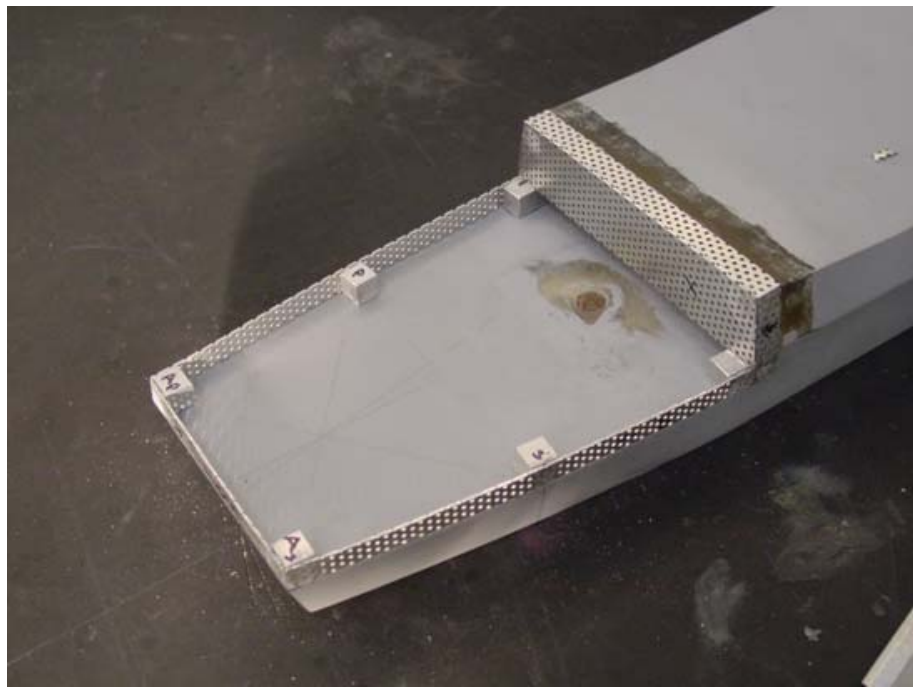

Figure 3.4. Construction of the raised flight deck and hangar extension. Note that the landing surface porous plate has not been installed yet.

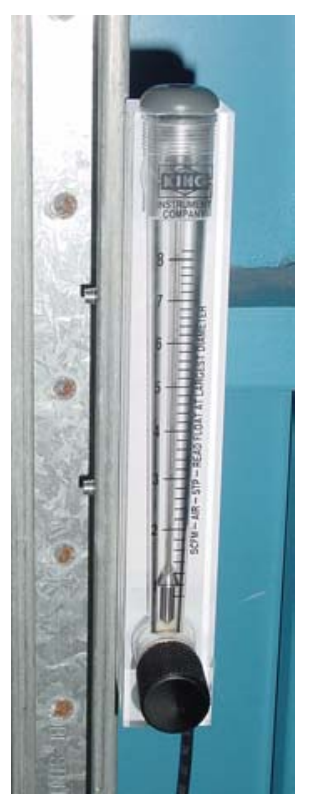

Figure 3.5. Float-type flow meter used to measure mass flow. 
Assuming constant density, the average velocity across a porous surface could be easily calculated from:

$$
\dot{m}=\rho A V
$$

The blowing configurations were tested with a mass flow of 4 cubic feet per minute, the maximum flow rate available with the setup. Given the area of the porous surfaces, the injection could ideally occur at less than $0.5 \%$ of freestream velocity across the entire surface. However, when the flow reached the plenum (the cavity below the porous surfaces), a uniform pressure distribution was difficult to obtain since the jet of air emptied into a relatively small volume. Two different techniques were used to solve this problem. The first technique used a circular baffle placed $1 / 4$ inch above the injection nozzle (Figure 3.6a). However, because the injected airflow acted on such a large surface area, the low injection velocity caused little effect to be noticed. Consequently, the other strategy was to pressurize only the hangar extension volume (Figure 3.6b). Given the area of this porous surface alone, the injection was $2.5 \%$ of freestream velocity. This

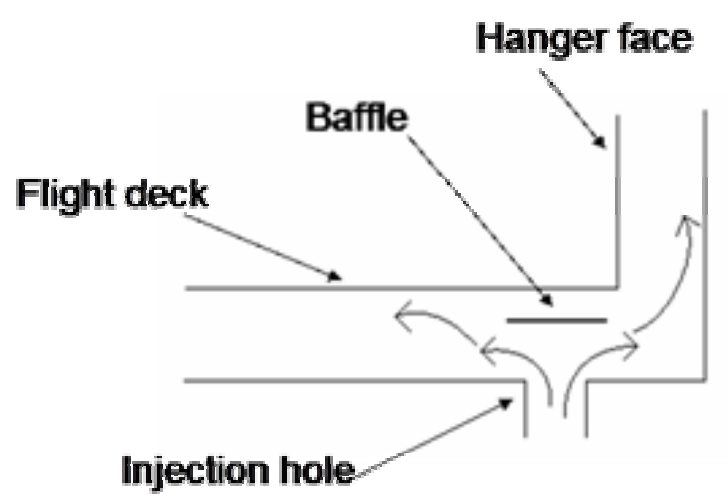

(a)

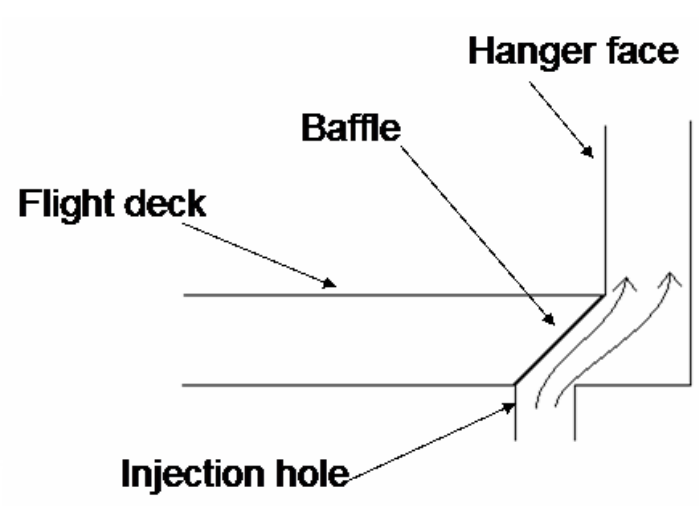

(b)

Figure 3.6. Side view of how injection is distributed in the cavities. The circular baffle (a) allows pressurization throughout the entire cavity. The diagonal baffle (b) channels all air to the hangar face. 
higher velocity had a greater effect on the flow, and because injection was in the direction of the freestream, momentum was added to help alleviate the velocity deficit.

The fences were also constructed of porous sheet metal and shaped with a variety of metalworking tools. Each fence had a height of $1 / 2$ inch. However, note that since some of these fences were angled 60 degrees upstream, they were only $1 / 4$ inch in vertical height. Finally, the commercially available reticulated foam was easily cut with scissors.

\subsubsection{Data acquisition system}

Thermal anemometry was used to acquire data. A single wire probe was used to determine the resultant velocity time history. A single wire probe has the disadvantage of having a velocity direction ambiguity but the advantage of sensing velocity without limitation to flow angle. This becomes particularly important behind objects that exhibit

bluff body separation and recirculation. The probe was oriented such that the resultant of the freestream and vertical components of velocity were measured. A Thermal Systems, Inc. IFA 100 Flow Analyzer and ThermalPro Software were used to acquire and analyze the data.

\subsubsection{Traverse system}

A Velamax two-component traverse was used to move the probe for data acquisition. Data was acquired from 17 inches above the boundary layer plate and 8 inches on either side of the model centerline for the ship yawed 0 degrees to the flow, see Figure 3.7a. With the ship yawed starboard 20 degrees, the grid was traversed across the tunnel from 16 to -5 inches, see Figure $3.7 \mathrm{~b}$. To capture the ship wake, a spatial 
resolution of one inch was used in the region behind the ship with an increased resolution directly downstream of the backward facing step as shown in Figure 3.7. The axial position analyzed was at the center of the flight deck, see Figure 3.8. This position was 3.0625 inches aft of the hangar face, or 3.0625h. Data was acquired at $4000 \mathrm{~Hz}$ (lowpass filtered to $2000 \mathrm{~Hz}$ ) for 4 seconds at each grid point. Because the ship wake effect on landing helicopters was the primary interest, the center of rotation of the ship (used to change yaw angles) was located near the center of the flight deck.

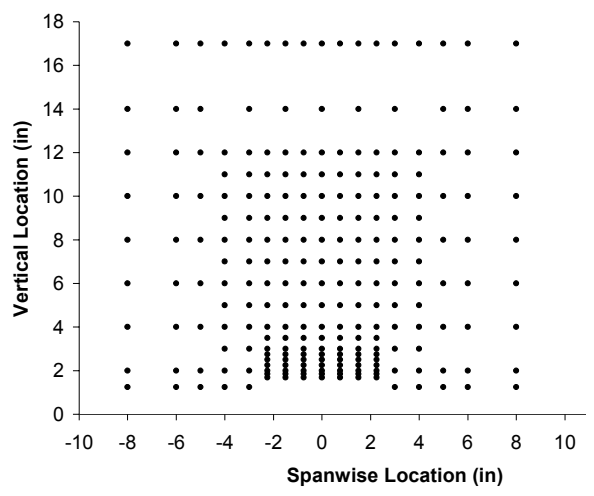

(a)

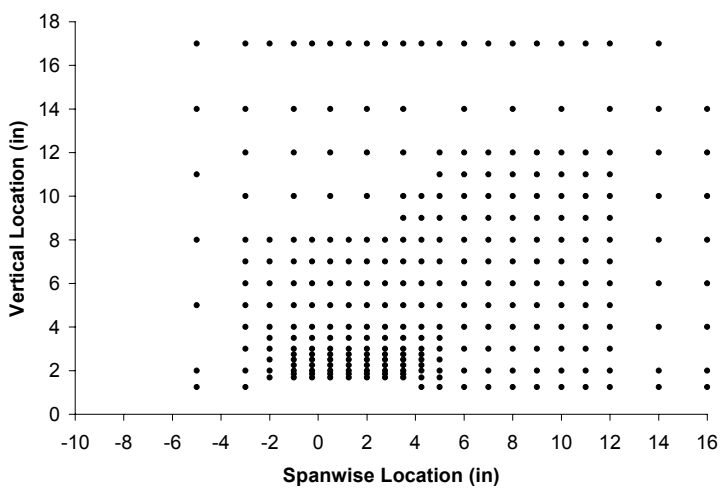

(b)

Figure 3.7. Experimental data grid at 0 degrees yaw (a), and 20 degrees yaw (b) as viewed looking upstream.

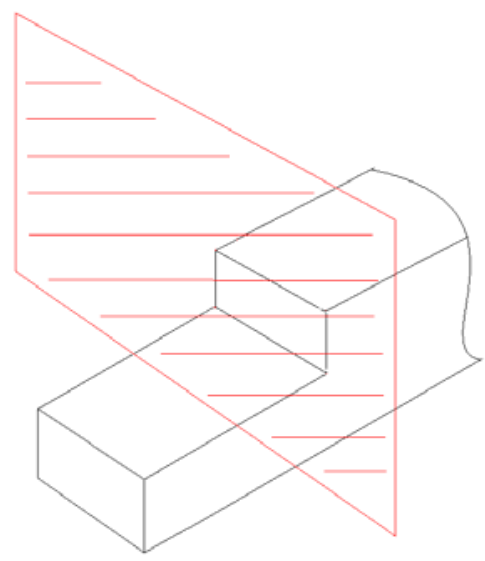

Figure 3.8. Sketch of the data plane. 


\subsubsection{Wind tunnel procedures}

The tunnel was allowed to thermally stabilize for at least 20 minutes before data acquisition commenced. The tunnel conditions were set to 75 feet per second, however, due to the blockage effect of the traverse system, and to a lesser extant, the boundary layer plate and mounting supports, the actual velocity near the model was slightly higher. Tunnel temperature stabilized at 78.3 degrees Fahrenheit. Tunnel test conditions (velocity, temperature, pressure, etc.) were acquired in addition to the thermal anemometry data. The number of data points acquired was 239 for the 0 degree WOD case and 287 for the 20 degree WOD case (Figure 3.7). The data were acquied in a topto-bottom, starboard-to-port pattern.

\subsection{Test plan}

Testing began by validating the setup with a tie-in run (Section 3.3.2). After this was completed, data were collected on the baseline configuration that included the solid raised flight deck and hangar extension as shown in the sketch in Figure 2.3. Note that the first 15 runs were conducted at 0 degrees wind-over-deck (WOD) angle. Refer to Table 3.1 for complete descriptions of the entire test.

The first objective of the test was to show that porous surfaces can be used to remove unsteadiness in the flow (run 3). Next, the effects of "venting" the porous flight deck were determined by comparing runs 3 and 5. Figure 3.9 shows the difference between theses two configurations. (Note: Run 4 was invalid due to an incorrect setup.) The size of the holes was also subject to investigation. As a result, the porous surfaces in run 6 had holes which were about $1 / 3$ the diameter of the prior porous material. Next, 
active configurations were considered by adding injection to the passive configuration which showed the most improvement. As seen in the run log, and will be seen in the results, the porous material used here had the smaller diameter holes, and the sides of the raised flight deck and hangar extension were solid (sealed with aluminum tape).

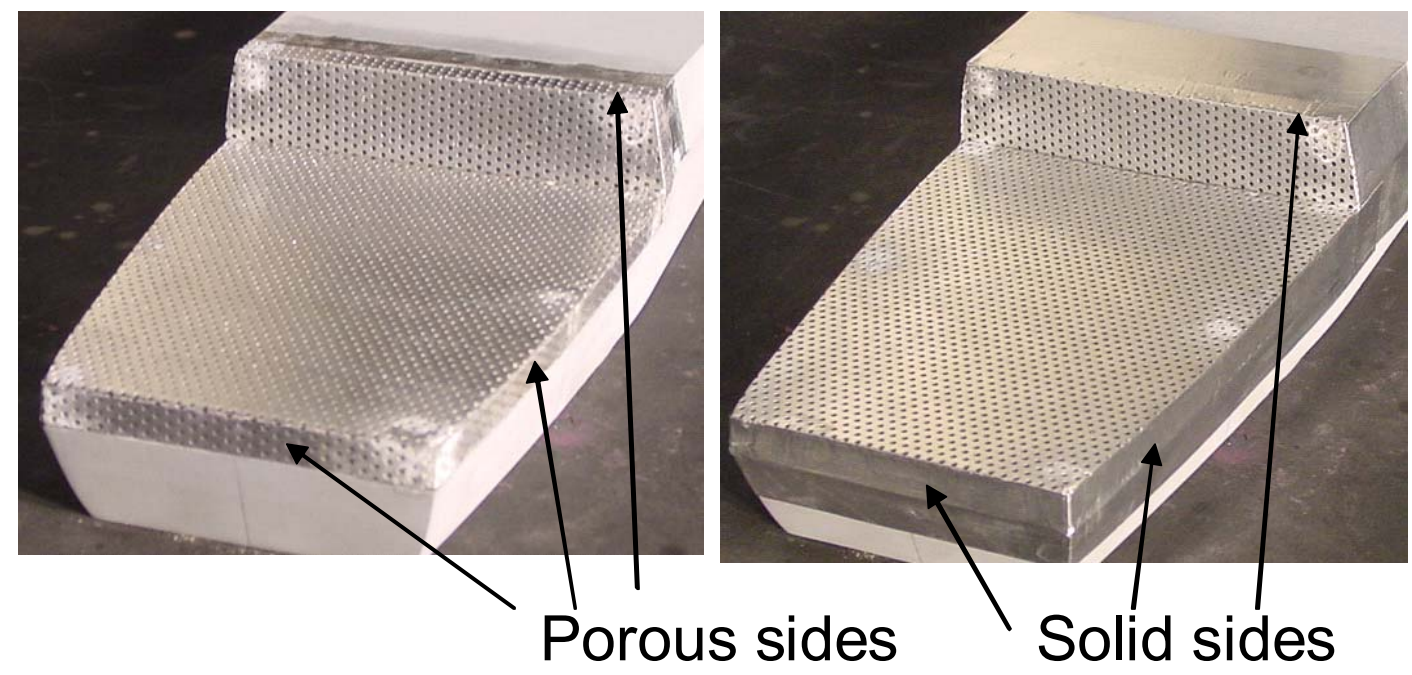

Figure 3.9. Differences between run 3 (left) and run 5 (right).

The active configurations investigated used two different strategies as mentioned above. First, in run 7, the circular baffle was tested. Then, the configuration which injected air only from the hangar face was tested in run 8 . Finally, run 9 determined how injection affects the flow if the flight deck is solid.

Upon completion of testing the configurations which used porous surfaces, data was taken again on the baseline model. There were two reasons for this. First, some concerns had arisen during prior runs with respect to random errors and the uncertainty of the hot wire measurement system. To remedy the situation, run 10 collected data at several grid points on the baseline configuration again. At each point, several time histories of differing length were recorded. Analysis of this data is found in Section 
3.3.3. The other purpose for this run was to verify that data is repeatable with the first baseline dataset.

Runs 11 to 15 collected data on the remaining configurations (the fences) introduced in Section 2.4. Motivation for using each device was stated above, as porous fences alleviate the increasing severity of the shear layers. Although most fences were porous, a solid version of the triangular-notched fence angled 60 degrees upstream ("mini delta wings") was tested. Constructing this device with solid sheet metal may better allow the formation of the leading edge vortices (run 14).

All wind tunnel runs discussed to this point were conducted at 0 degrees WOD. Realizing that the most difficult conditions for helicopter operations are not necessarily at this flow angle, a limited number of runs were conducted at 20 degrees WOD. Due to time constraints, only two configurations, besides a new baseline, were tested. As a result, the configuration which showed the most improvement using porous surfaces was chosen, along with the most favorable fence configuration. 


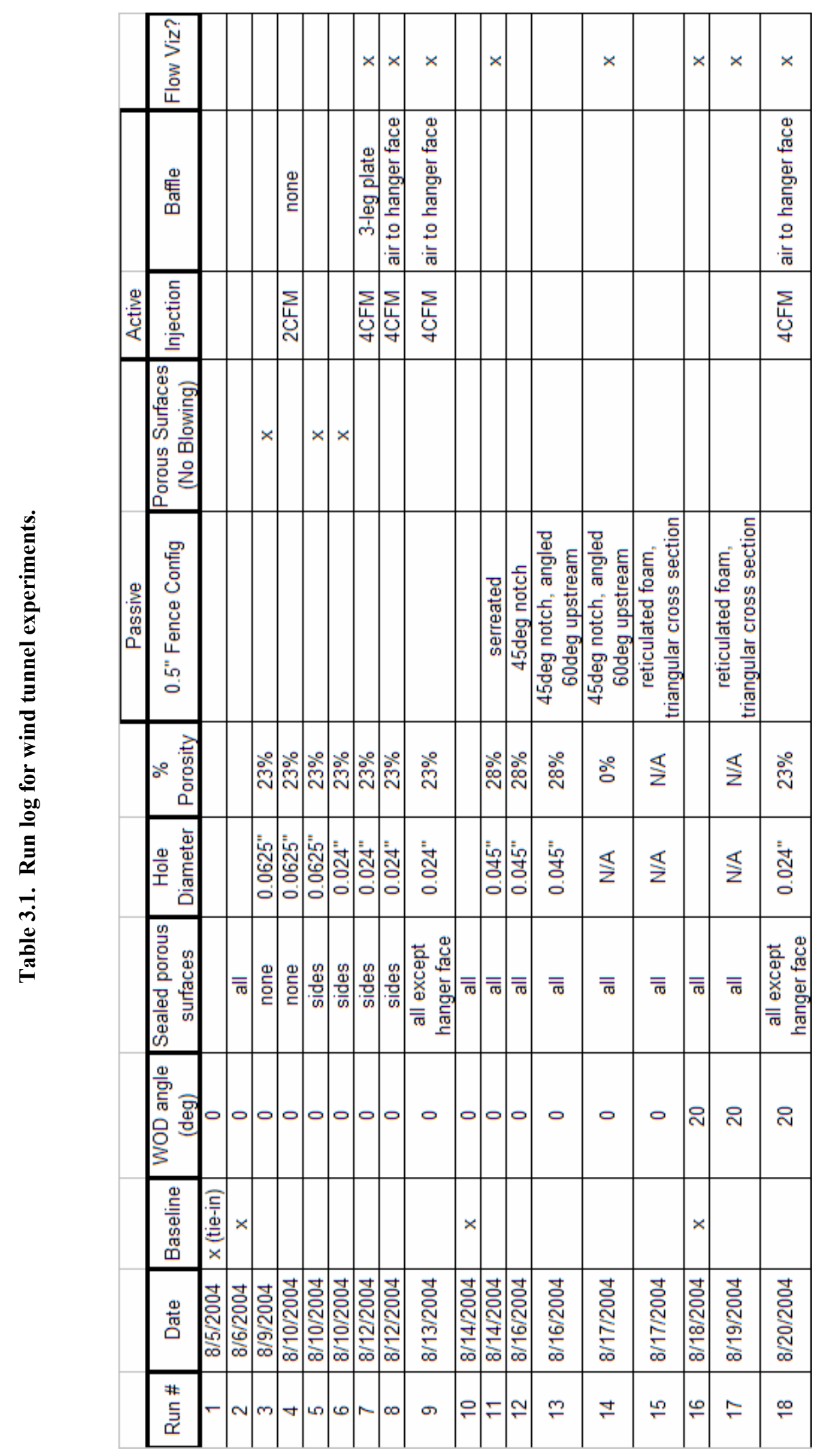




\subsection{Uncertainty / repeatability}

\subsubsection{Measurement uncertainties}

The percent error of the hot wire calibration was found to be under $+/-1 \%$ (except at a tunnel velocity of 50 feet per second where it was found to be $+1.23 \%$ ). Tunnel temperature variations were +/-0.2 degrees Fahrenheit. As mentioned previously, the tunnel velocity spatial uniformity is approximately $1 \%$. In addition, the tunnel thermocouple was estimated to be accurate within +/-0.1 degrees Fahrenheit. The ambient pressure was accurate to $+/-0.005$ inches of mercury. A sensitivity analysis revealed that temperature affected the calculated velocity at 0.53 feet per second per degree Fahrenheit. Similarly, the pressure sensitivity was -2.6 feet per second per inch of mercury. The total experimental uncertainty was determined in a method outlined by Rae and Pope $\mathrm{e}^{35}$ and the measured velocity was estimated to be within $+/-1.3 \%$. This estimate is valid for flows with low turbulence intensities. Because the backward facing step flow has large values of turbulence intensity, additional errors with the hot wire measurements exist in this investigation. Bruun ${ }^{36}$ shows that for a turbulence intensity of $30 \%$, a hot wire over-predicts the mean velocity by about $5 \%$ and under-predicts the fluctuating components by about $10 \%$ of the mean. Above $30 \%$ turbulence intensity (which corresponds to about $20 \%$ measured), the measurements may become misleading. Therefore measurements in regions where the turbulence intensity is greater than $20 \%$ should be analyzed qualitatively only. Finally, the mass flow meter had an uncertainty of $+/-3 \%$ of full scale deflection. At 4 cubic feet per minute, this yields an uncertainty of $+/-$ $6 \%$. 


\subsubsection{Tie-in run}

To validate that the setup was functioning properly, testing began with a baseline configuration that had been tested in the past. Figure 3.10 shows the velocity standard deviation contours from prior experiments and the data measured in the current experiments. The configuration and tunnel conditions were the same. Because these resulting contour plots are almost indistinguishable, concerns with repeatability were minimized.

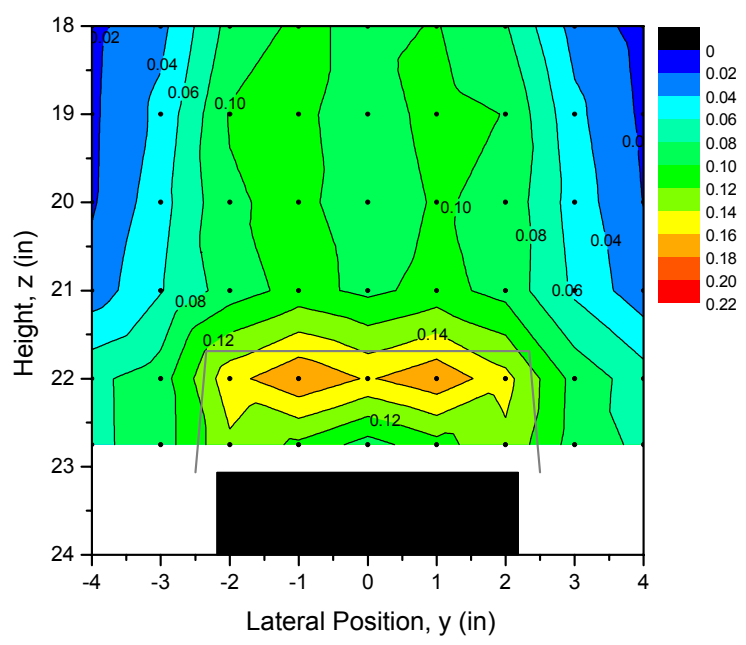

(a)

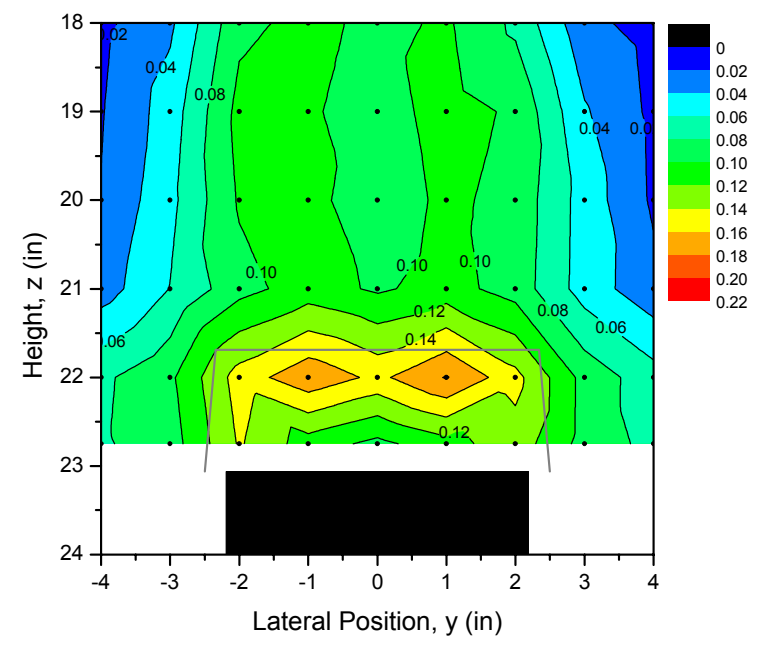

(b)

Figure 3.10. Velocity standard deviation above the center landing spot for a baseline model during (a) prior experiments and (b) current experiments.

\subsubsection{Measurement system random uncertainty}

During the experiment, run 10 was dedicated to documenting the random uncertainty of the measurement system. The motivation for this analysis occurred when it was noticed that multiple 4 -second samples at the same grid point sometimes yielded different statistics (mean velocity, standard deviation, etc.). These differences were often on the order of a few percent. As a result, it was necessary to document this randomness. Ten points were selected at a variety of locations (see Figure 3.11). Using information 
obtained in earlier runs, the mean velocity and standard deviation varied widely at the selected grid points. Eight separate 4-second samples and two 32-second samples were taken at each grid point. Independent 4-second samples were compared to determine how well different samples agree with each other. The 32-second samples allow running averages to be computed to determine how much data must be recorded to truly calculate the mean flow. Note that it is difficult to determine what length signal yields a completely ergodic flow due to the inherent uncertainties in any experiment.

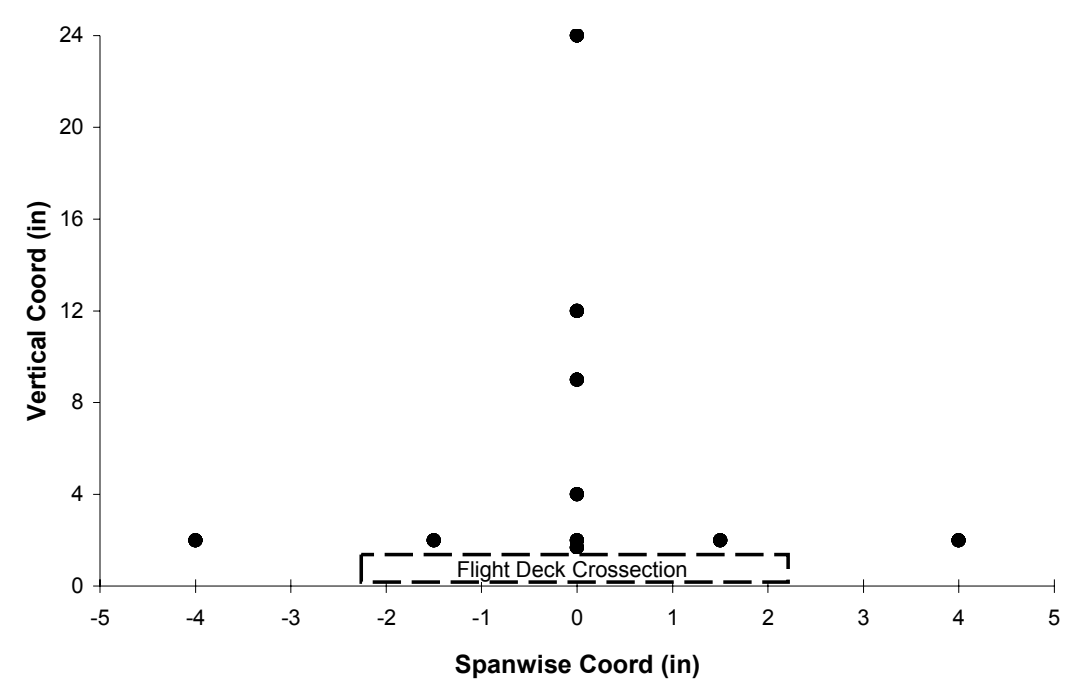

Figure 3.11. Grid locations used to document the measurement system's random error.

First, consider signals in the freestream. Figure 3.12 shows eight independent 4second velocity time histories. Although the signals appear to have reached a steadystate value around 3 seconds, the different signals still vary by approximately $+/-0.1 \%$. This is acceptable because the $1.3 \%$ uncertainty stated above is more significant than this smaller random error. However, Figure 3.13 shows that low uncertainty is not achieved at grid points located deep within the airwake. The location $y / b=0, z / h=0.34$ is much 
more unsteady, resulting in a larger uncertainty. Although the running averages are not nearly as smooth here, the random uncertainty is approximated at $+/-0.5 \%$ of freestream or $+/-2.7 \%$ of local mean velocity. This error is now more than twice the size of the stated uncertainty. The probable explanation for this increase in error is due to the high turbulence intensity at this spatial location. As a result, sampling time was increased to determine if the error were random and if it could be reduced. However, Figure 3.14 shows that two independent 32-second samples still do not converge to the same value. Given the tunnel speed and model size, 32 seconds should be a sufficiently long time for the flow to become ergodic. Additionally, it is noted that the flapping frequency ${ }^{7}$ of the backward facing step flow in this investigation is 90 hertz. Acknowledging that some frequency content still exists at a frequency one order of magnitude smaller, a 4-second sample (and surely a 32-second sample) should still provide a long enough sample to be statistically confident. Therefore, it was concluded that discrepancies in the mean velocity of independent signals were mainly due to the random errors of the hot wire measurement system, and that a 32-second sample provided only a slightly better measurement than the 4-second sample. Thus, this improvement was not worth the extra run time and data storage requirements. 


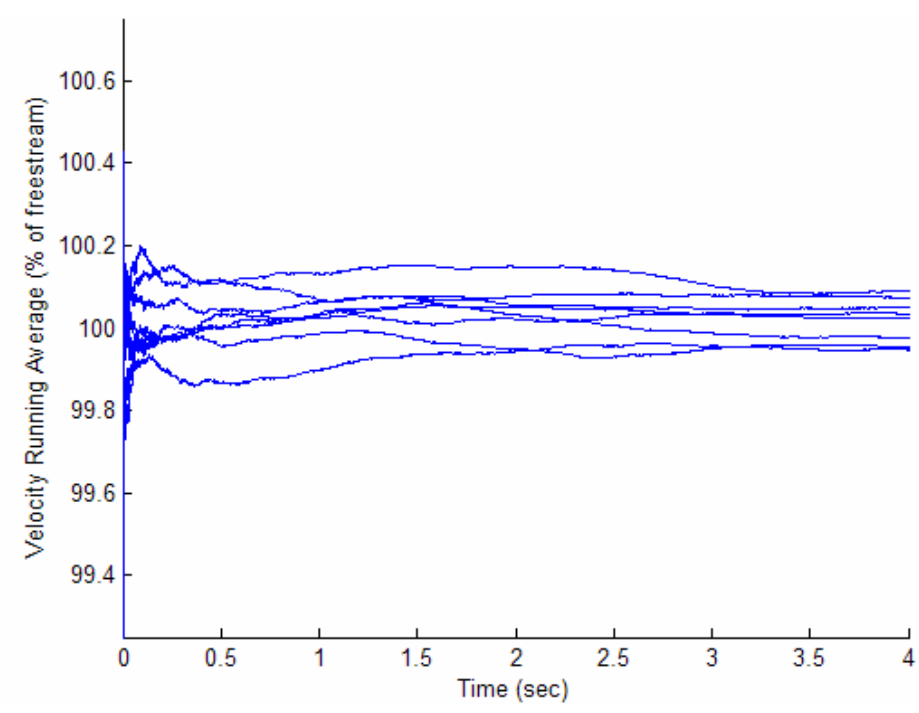

Figure 3.12. Velocity running average of independent time histories at a single spatial location in the freestream.

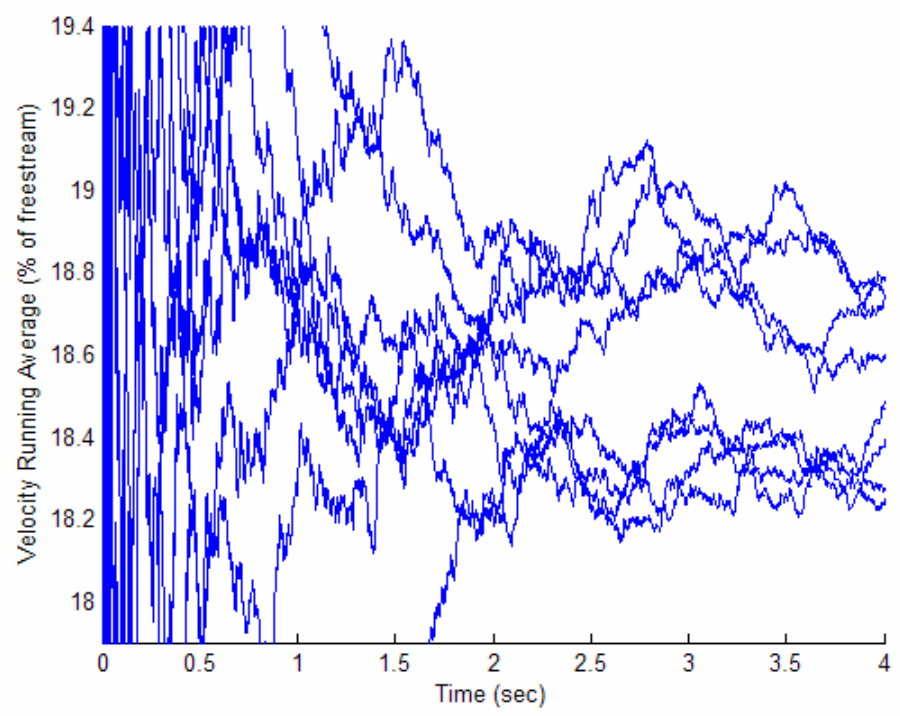

Figure 3.13. Velocity running average of independent time histories measured on the centerline at $z / h=0.34$. 


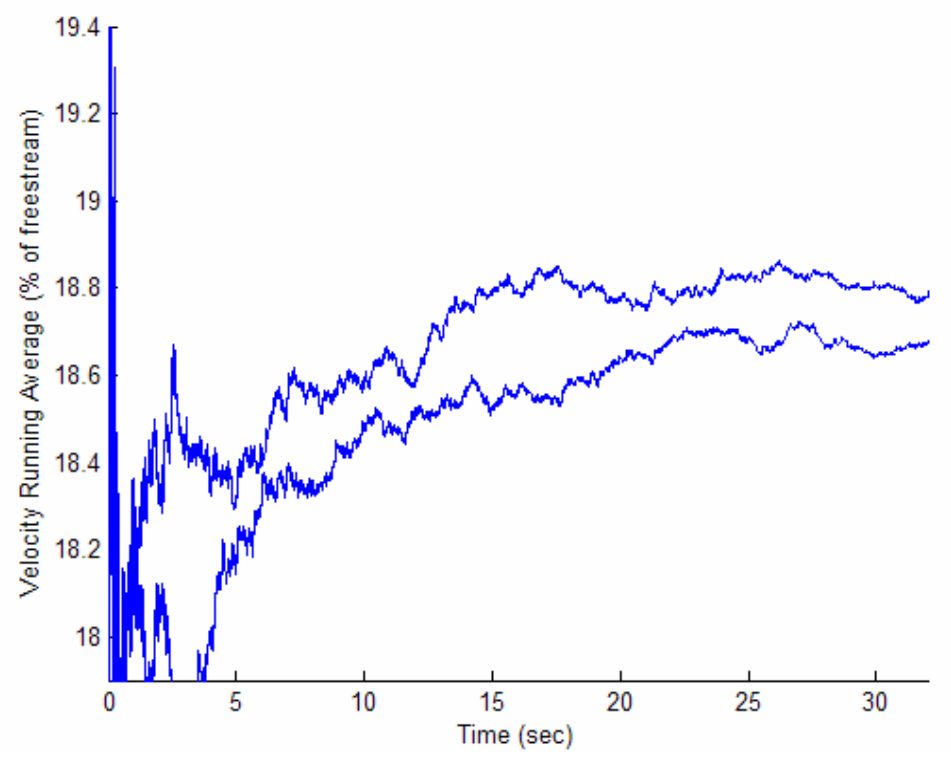

Figure 3.14. Velocity running average of independent 32-second time histories measured on the centerline at $z / h=0.34$.

A similar uncertainty analysis was completed on the calculated standard deviation values. It was found that the freestream standard deviation value has a random error of $+/-1.3 \%$. In the unsteady recirculation region $(y / b=0, z / h=0.34)$, standard deviation values were found to vary by $+/-4.0 \%$. Again, these figures were calculated by comparing the standard deviation of the eight independent 4-second time histories measured at a single grid point. 


\section{RESULTS AND DISCUSSION: 0 DEGREES WIND-OVER-DECK}

The goal of this investigation is to find flow control devices which improve the flow field over the rear landing deck on frigate-type ships. Helicopter/ship operations must be conducted at a variety of wind-over-deck (WOD) angles, but to remove as many experimental variables as possible, most of the wind tunnel runs were conducted with the ship centerline parallel to the tunnel centerline ( 0 degree WOD). This chapter analyzes these flow conditions, and the following chapter analyzes the limited number of runs at 20 degrees WOD.

\subsection{Mean velocity}

Mean flow is a basic method of comparison for the flow control devices. Examining mean velocity profiles is useful because changes in the mean flow can cause increased pilot workload. Additionally, knowledge of the airwake shape and location of greatest velocity deficit helps understand the physics of the flow field. Recall from the uncertainty analysis that the measured mean velocities in regions of high turbulence intensity (typically below one hangar height) should be analyzed qualitatively only.

\subsubsection{Mean velocity contours}

A cross-section of the baseline model is shown in Figure 4.1. The measurement plane was perpendicular to freestream at a longitudinal position above the center of the 
landing deck (recall Figure 3.8). On the left, the entire flow field is shown (Figure 4.1a); an enlarged view near the backward facing step is on the right (Figure 4.1b), shown by dashed lines in (a). Because the experimental data grid (Figure 3.7) did not have equally spaced points, a uniform plotting grid had to be created to draw contour plots. As a result, a new grid with much smaller spacing was created using linear interpolation between the experimental data (see Appendix C). The black rectangle represents the original DDG model and the gray rectangle represents the raised flight deck.

Additionally, an outline of the hangar face (a gray line) is included for reference. All plots in this section have the same contour levels and colors, ranging from $0 \%$ (blue) to $104 \%$ (red) of the freestream velocity. The grid points are also shown for reference.

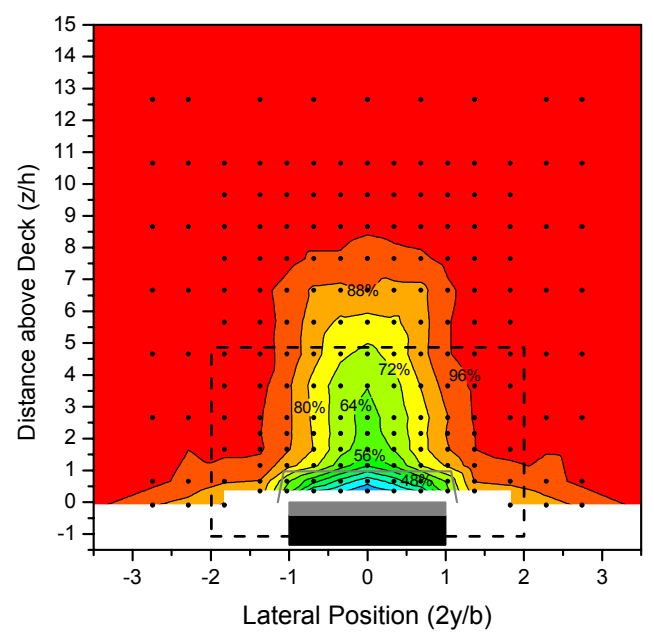

(a)

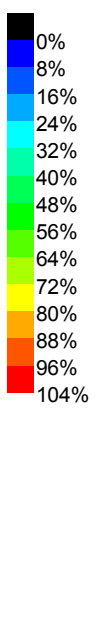

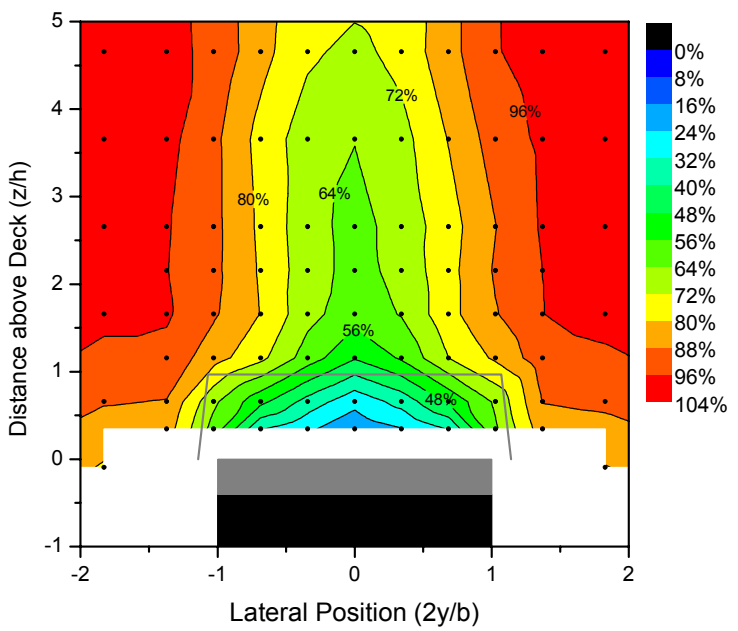

(b)

Figure 4.1. Mean velocity of the baseline configuration (contours in \% freestream); (a), the entire flow field, and (b), the region of interest.

A steep velocity gradient exists downstream of the hangar, and it extends as far at the measurement system can traverse vertically. Figure 4.2 shows the effect of replacing ship surfaces with porous surfaces. Although the data plotted here is from run 3 , runs 5 and 6 appear to be almost identical to run 3. (Refer to Table 3.1 for details on the 
specific configurations of each run.) An easier comparison between configurations can be made with velocity profile line plots, included in the next section. The mean velocity gradient downstream of the hangar in run 3 is larger because the contour lines are closer than for the baseline configuration. This phenomenon alone may increase workload for the pilot, however, other factors, such as flow unsteadiness, must be considered. This is discussed in detail in the remaining sections of this chapter.

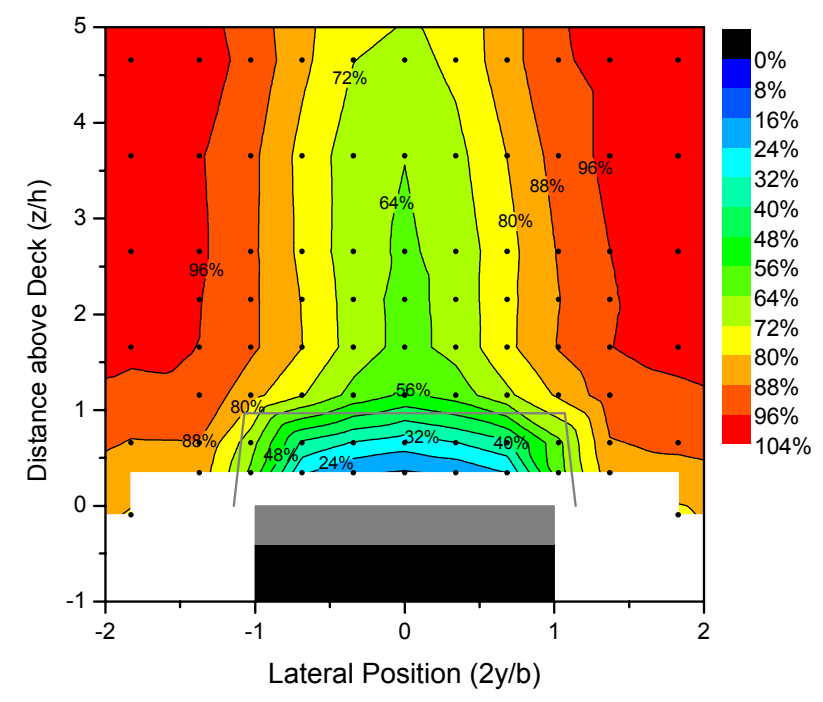

Figure 4.2. Run 3 mean velocity.

Figure 4.3 shows the mean velocity contours of run 8 . This configuration included injection from the hangar face. The mass flow rate was four cubic feet per minute which translates to an injection velocity of 1.9 feet per second or $2.5 \%$ of the freestream velocity. This configuration caused another increase in the mean velocity gradient. The other two configurations which incorporated blowing (runs 7 and 9) have mean velocity contours that do not differ as significantly with respect to the baseline. 


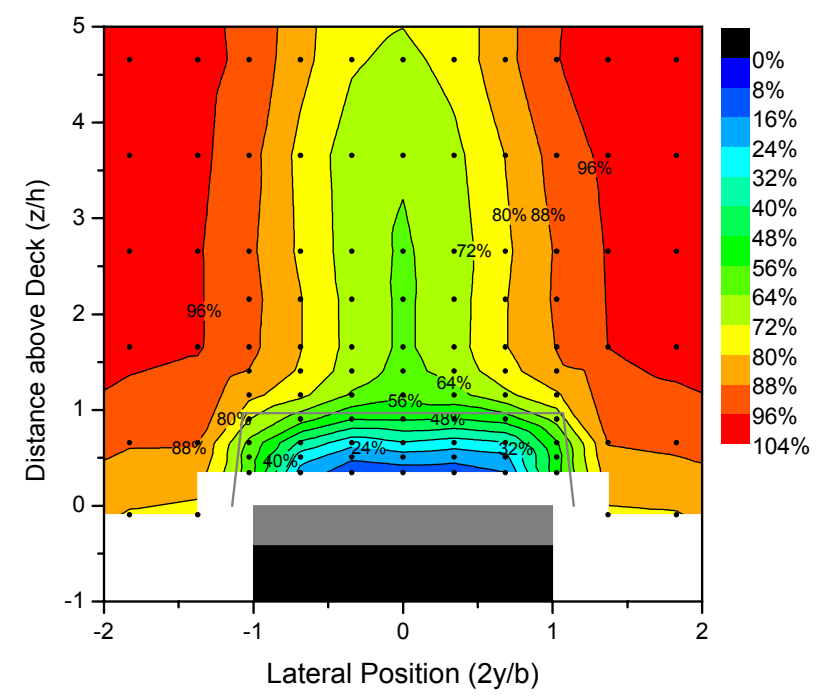

Figure 4.3. Run 8 mean velocity.

Next, consider the different fence configurations. Figure 4.4 shows the mean velocity using the porous serrated fence. There is no longer a steep velocity gradient downstream of the hangar. Instead, the velocity changes rapidly beyond the edge of the fence. Figure 4.5 shows a slightly less severe gradient with the triangular-notched porous fence (run 12). Runs 13 and 14 exhibit almost identical mean velocity contours. Because the frontal areas of these fences are smaller, the gradient is not pushed as far away from the hangar (see Figure 4.6). Finally, Figure 4.7 shows the mean velocity contours using the reticulated foam fence (run 15). This configuration has similar results with runs 11 and 12 , however, run 15 produced the largest region of low speed flow downstream of the hangar. 


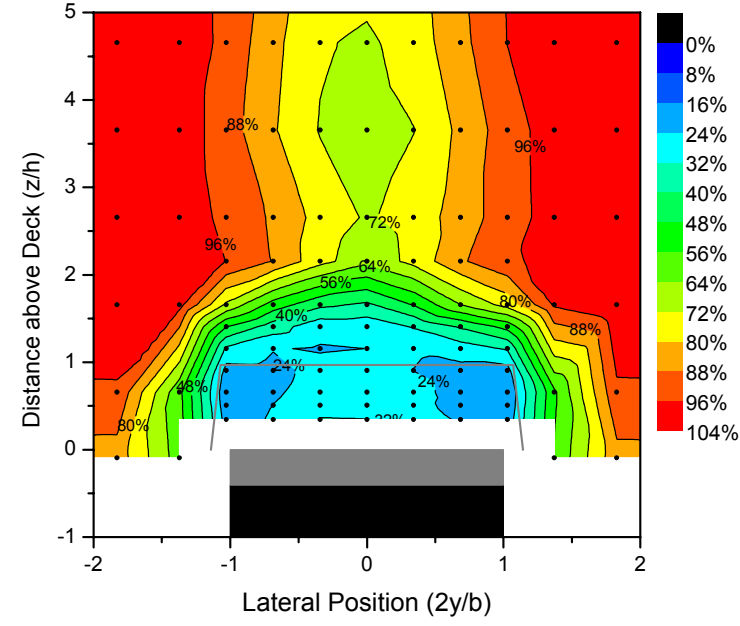

Figure 4.4. Run 11 mean velocity.

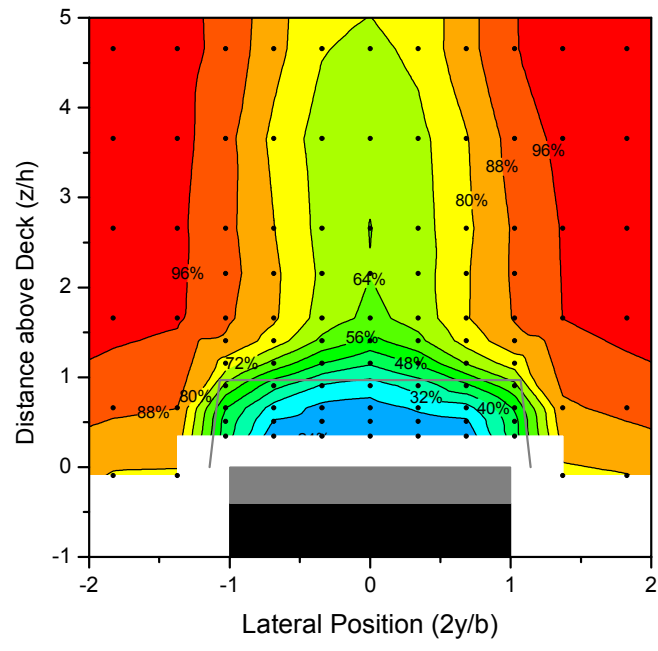

Figure 4.6. Run 13 mean velocity.

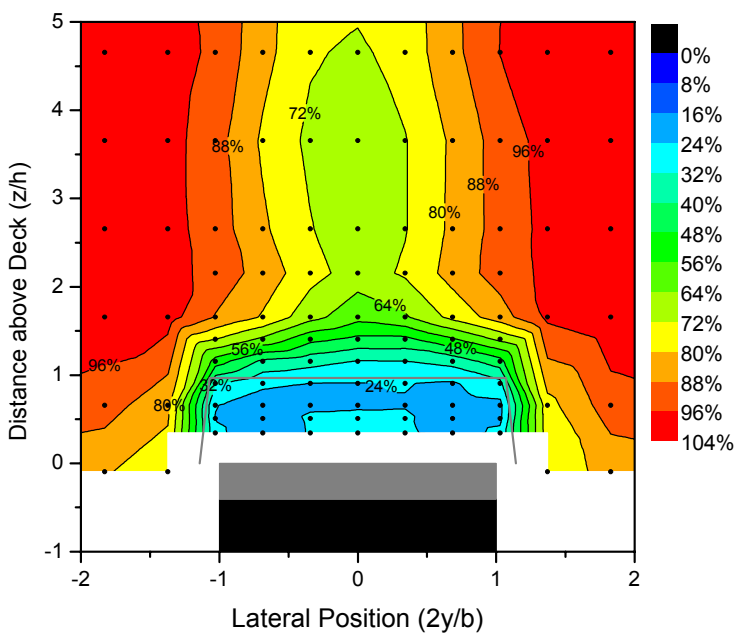

Figure 4.5. Run 12 mean velocity.

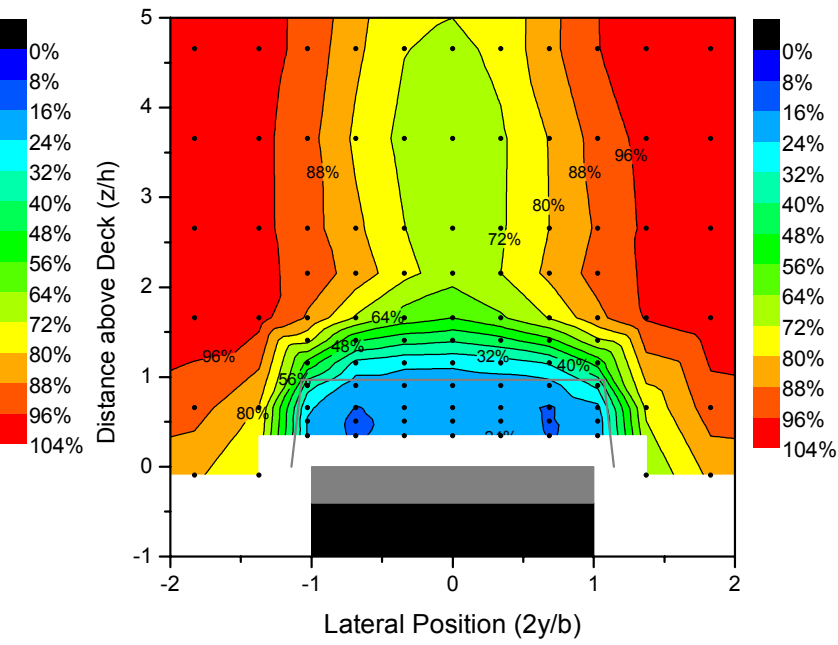

Figure 4.7. Run 15 mean velocity.

\subsubsection{Mean velocity line plots}

Vertical and horizontal slices of the contour plots serve as an easy means of comparison between configurations. Although different horizontal heights can be examined, a line plot at rotor height during a low hover (a few feet above the deck) is considered to be an important location in the flow field. This height is typically a small distance below the top of the hangar. In this investigation, a vertical height of $z / h=0.66$ is used for analysis. Figure 4.8 shows results from the porous ship surface 
configurations. The use of porous ship surfaces causes a decrease in the mean flow across the flight deck. This may be a desirable effect since landing at higher velocity means that a helicopter must be pitched downward to maintain a stationary position with respect to the ship. Alternatively, this can be considered a negative effect if increased hover performance is required. With an increased oncoming velocity, a helicopter may be able to take off at higher gross weights. However, due to the limitations of the single component hotwire, the mean velocities plotted here could come from any direction in the vertical/longitudinal plane. The asymmetric feature in run 7 (shown in Figure 4.8) results from a non-uniform injection velocity across the flight deck surface. Recall that the circular baffle did not distribute the airflow uniformly.

The mean velocities for the fence configurations are shown in Figure 4.9. These configurations show a larger decrease in mean velocity than the porous surface configurations. The reticulated foam fence configuration (run 15) shows the lowest mean velocity across the flight deck. However, there is a tradeoff with this improvement; a steeper velocity gradient exists beyond the lateral edges of the ship (from $|2 y / b|=1$ to $|2 y / b|=1.5)$. As a result, if the pilot deviates laterally from an approach, he/she will be caught in a steeper gradient than occurs for the baseline configuration. 


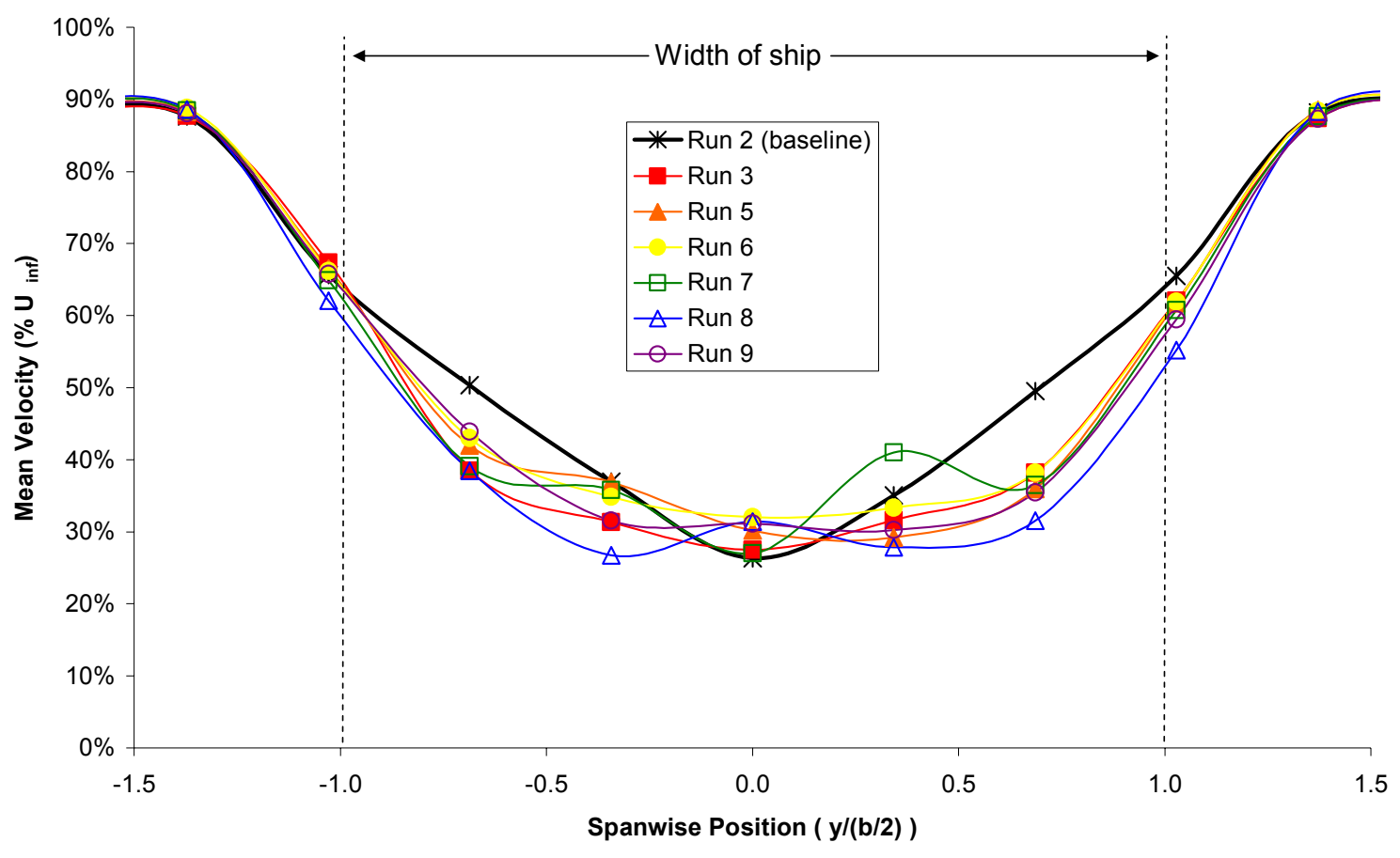

Figure 4.8. Mean velocity at low hover $(z / h=0.66)$ for porous surface configurations

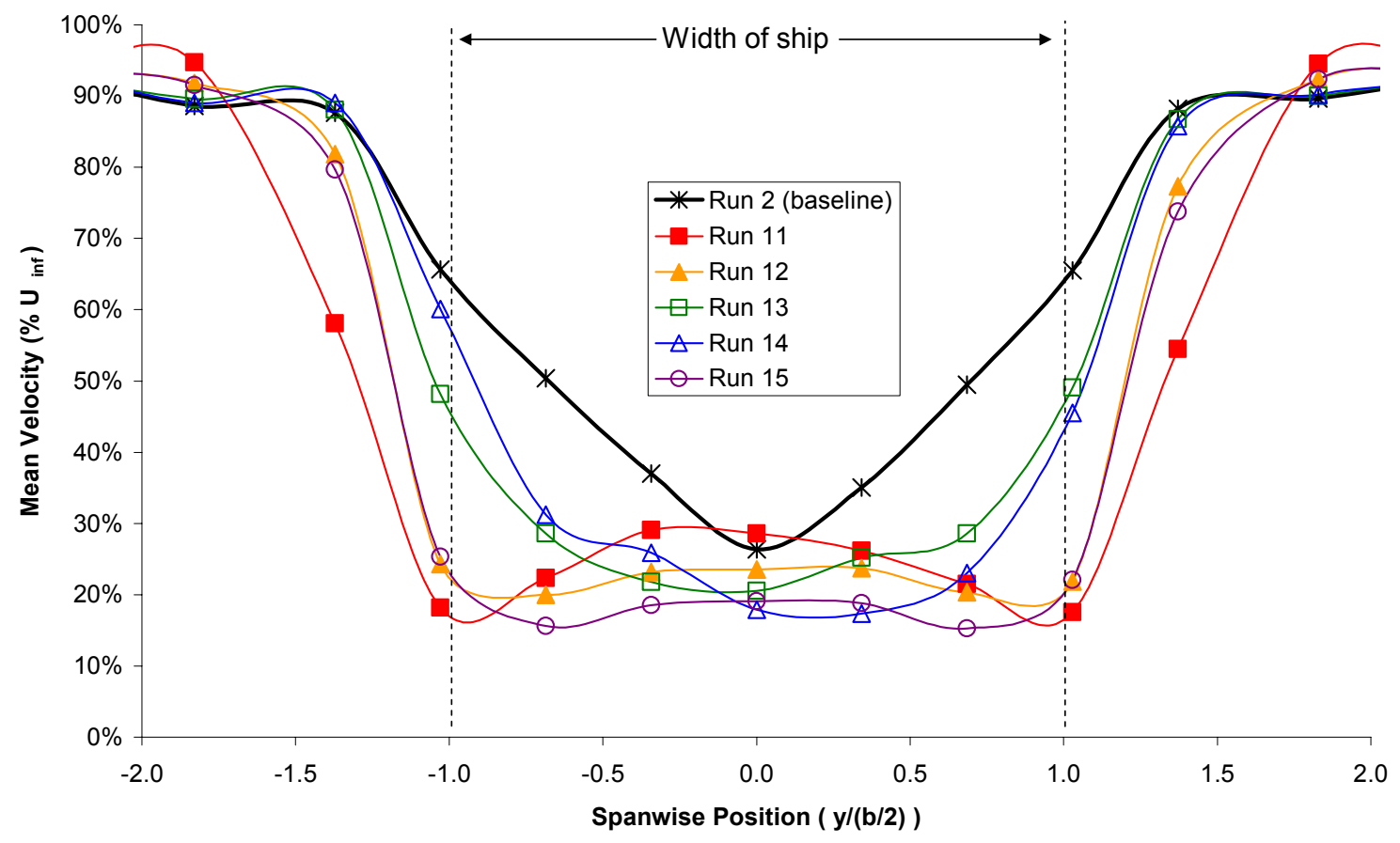

Figure 4.9. Mean velocity at low hover $(z / h=0.66)$ for fence configurations. 


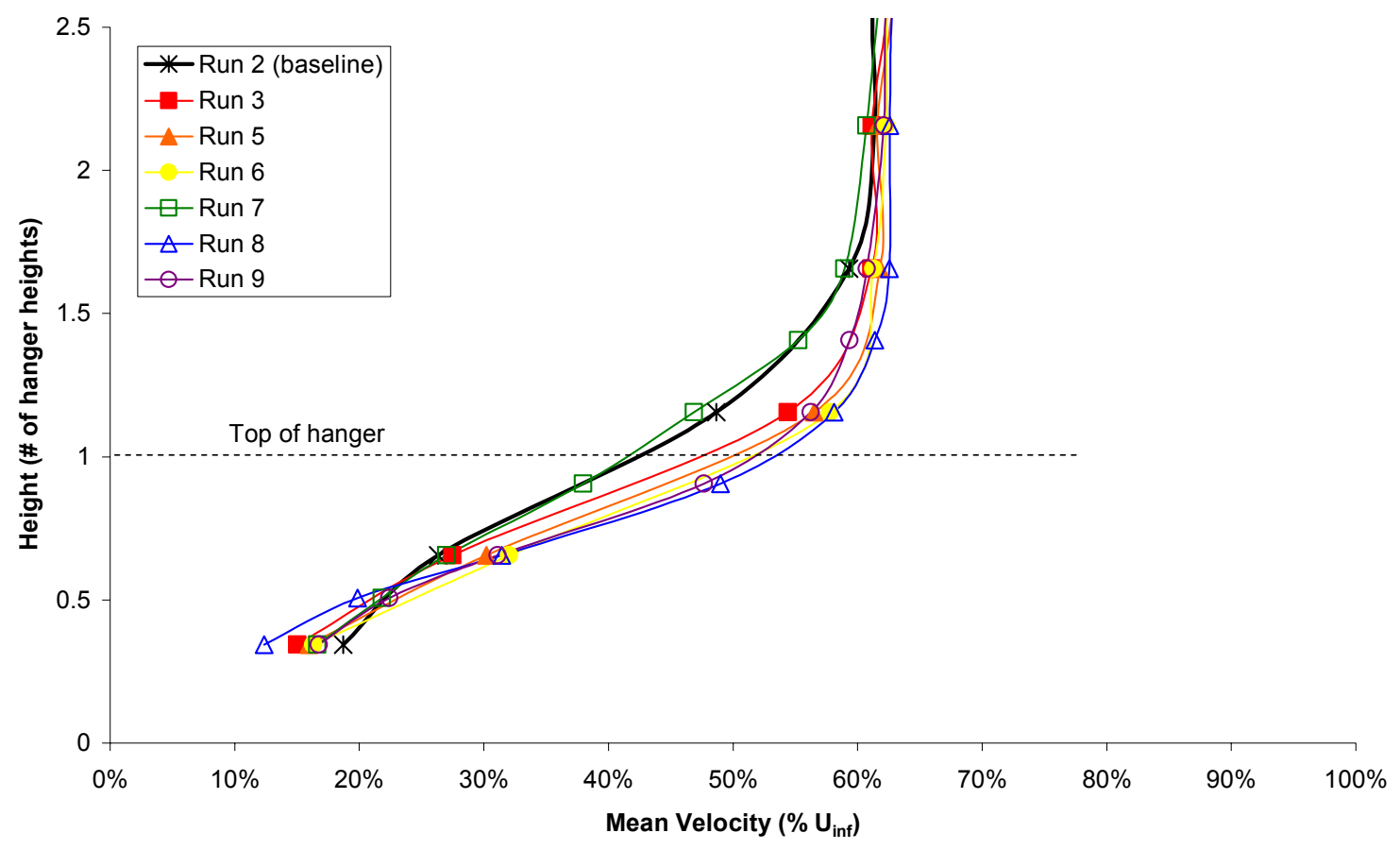

Figure 4.10. Mean velocity on the vertical centerline $(2 y / b=0)$ for porous surface configurations.

A vertical cut of the mean velocity contour plots also helps show quantitative differences between configurations. Figure 4.10 plots the mean velocity at various heights above the deck. Although there is little variance in the shape of these lines, most configurations cause a slight increase in velocity. Recall from above that these configurations tend to increase the velocity gradient behind the hangar, an undesirable feature. The fence configurations produce a very different effect on the mean velocity and are shown in Figure 4.11. Fences "shield" the flight deck. The serrated fence (run 11) has the most dramatic effect, keeping the velocity around $30 \%$ of freestream until about 1.5 hangar heights. The other configurations have a similar effect, but do not keep the mean velocity as low at heights above the top of the hangar. However, these other configurations do have lower mean velocity near the flight deck compared with the porous surface configurations. 


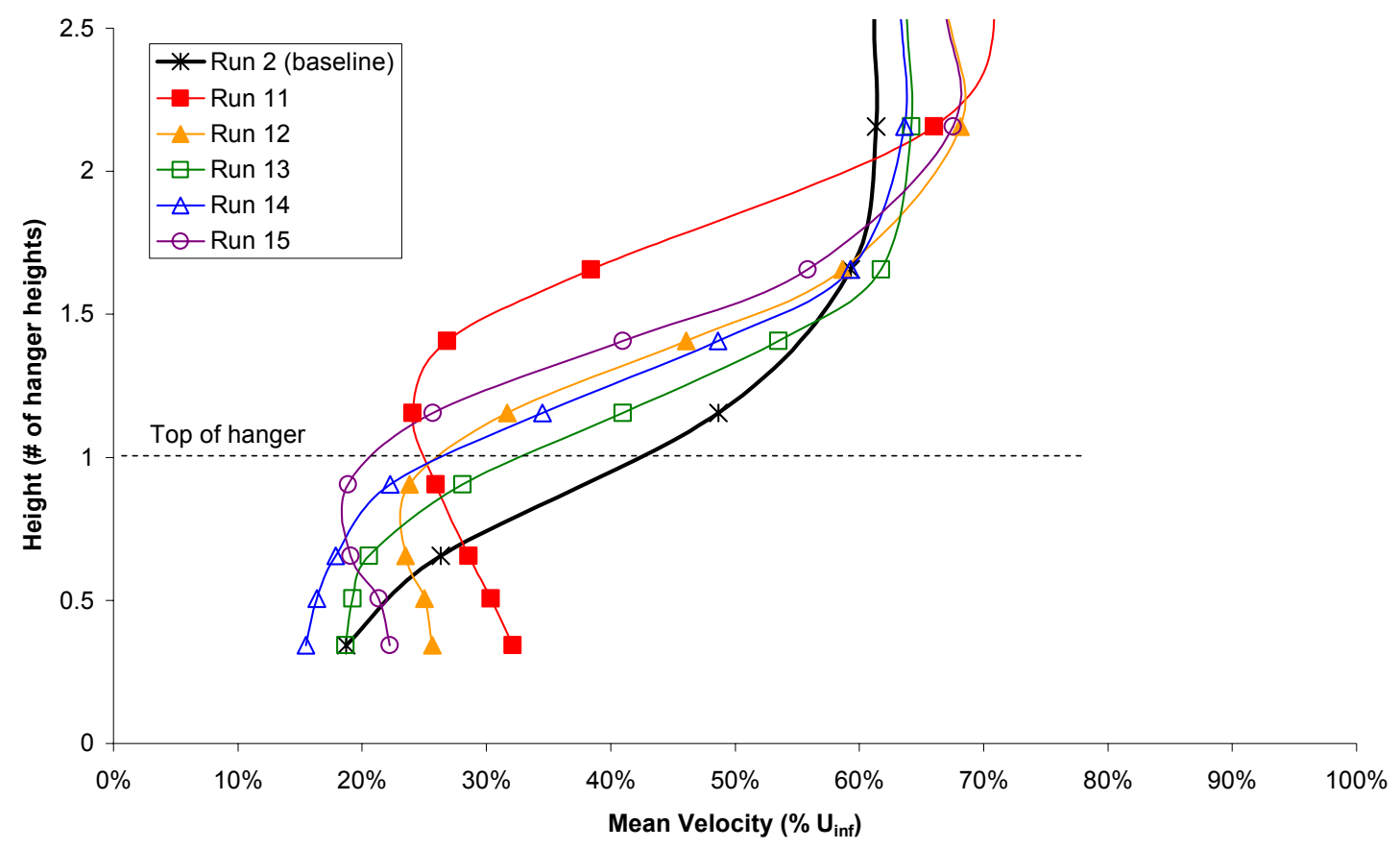

Figure 4.11. Mean velocity on the vertical centerline $(2 y / b=0)$ for fence configurations.

In summary, it has been shown that the configurations tested generally result in lower mean velocities near the flight deck. Acknowledging that it may be desirable to actually increase the velocity over the flight deck in some situations, the mean velocity gradients are of more concern with respect to pilot workload. Because the lower velocities near the deck must recover to freestream in the farfield, steeper gradients occur when the velocity near the flight deck is lower than in the baseline. Accordingly, it must be determined if the velocity reductions behind the hangar are worth the danger of larger velocity gradients above and to the sides of the helicopter's landing region.

Compromising between the velocity reduction and increased gradients, the reticulated foam fence (run 15) is the most successful device out of all the fence configurations, while injection from the hangar face (run 8) is the most successful configuration which utilizes porous ship surfaces. 


\subsection{Velocity standard deviation}

Although the mean flow variation over the landing deck provides a basic understanding of the varying behaviors of different control techniques, it does not provide information about the unsteadiness of the flow field. Since random fluctuations are correlated with increased pilot workload, ${ }^{23}$ a measure of unsteadiness should be calculated to determine the effectiveness of flow control devices. Consequently, the standard deviation of the velocity time history at each grid point is computed. Although mean velocity gradients can be a contributing cause to pilot workload, the unsteadiness in the flow field is used as the primary metric for evaluation. For small fluctuations, standard deviation, $\sigma$, is the RMS of the velocity fluctuations in the direction of the measured resultant velocity. Recall that because a single component hotwire probe is used, only the resultant of the freestream and vertical components is measured. A more thorough discussion is available in Appendix A and B.

The contour plots that follow include all the configurations tested. The unsteadiness from different configurations varies more from the baseline than the mean velocity. The contours show the location of the greatest unsteadiness in the flow field and provide insight on how to further decrease unsteadiness. Recall from the uncertainty analysis that the measured velocity standard deviation in regions of high turbulence intensity (typically below one hangar height) should be analyzed qualitatively only.

\subsubsection{Velocity standard deviation contours}

Perhaps the easiest way to get a general idea of how each flow control technique performs is to examine contour plots of the velocity standard deviation at each grid point. 
The baseline configuration is shown in Figure 4.12. The left plot shows the entire flow field, and the area of interest is enlarged on the right. Again, the black rectangle represents the original model, the gray rectangle represents the raised flight deck, and the outline of the hangar face is represented by a gray line. The hotwire grid points are also shown for reference. All plots have the same contour levels and colors, ranging from $0 \%$ (blue) to $22 \%$ (red) of the freestream velocity.

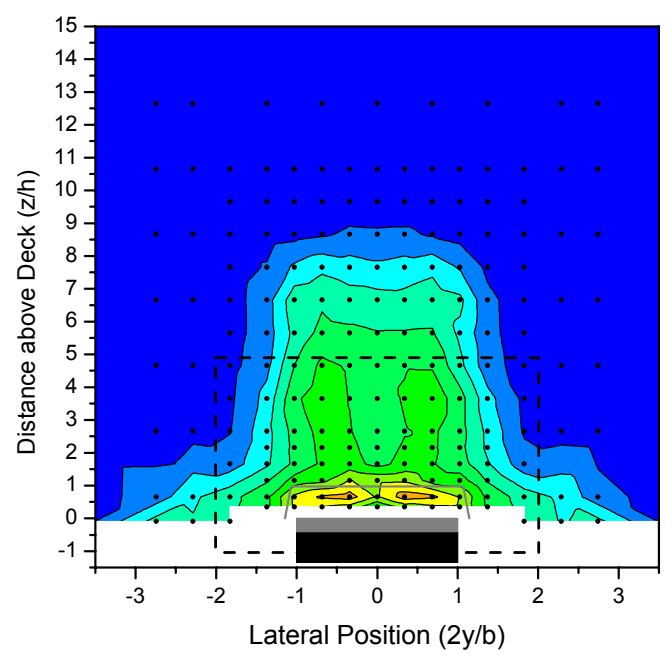

(a)
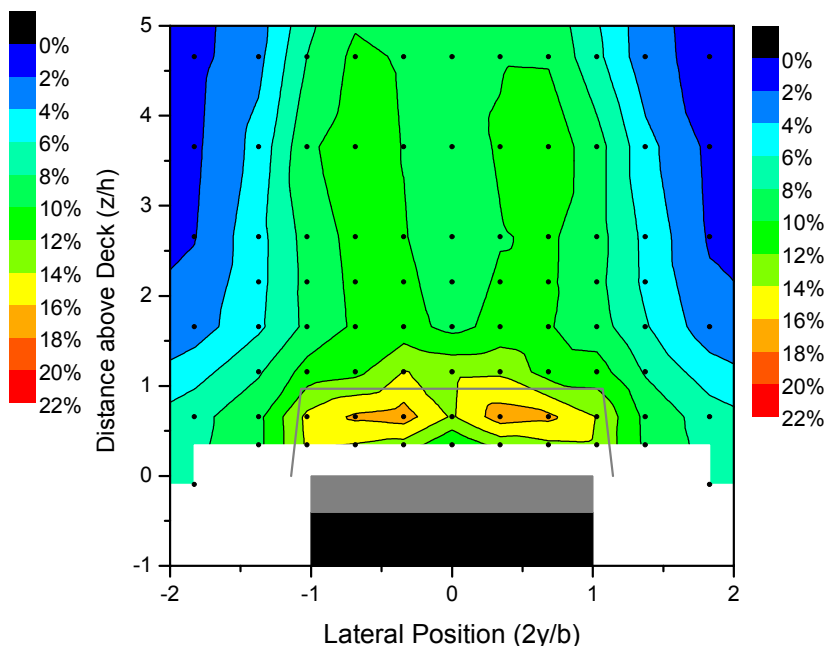

(b)

Figure 4.12. Velocity standard deviation of the baseline data; (a), the entire flow field, and (b), the region of interest.

Figures 4.13 and 4.14 show the contour plots which compare the effect of "venting" the raised flight deck and hangar extension. Figure 4.13 is vented while Figure 4.14 is not. Both of these configurations show a slight reduction in maximum standard deviation compared to the baseline. Upon closer inspection, it is seen that the non-vented case has slightly less unsteadiness. As a result, the remainder of the porous surface configurations did not incorporate venting; the sides of the raised flight deck and hangar extension are solid. The effect of hole diameter is determined in Figure 4.15. The holes 
in the porous surfaces in this configuration are 0.024 inches in diameter, as opposed to 0.0625 inches in the prior runs. Both materials had 23\% open area. Little change occurs, but a possible explanation is due to the vertical limit on the probe/traverse.

Measurements were only possible down to a height of 11/32 inch above the flight deck surface, and it is likely that greater differences occur within this region. Regardless, it was decided to move forward with the investigation using the material with smaller holes.

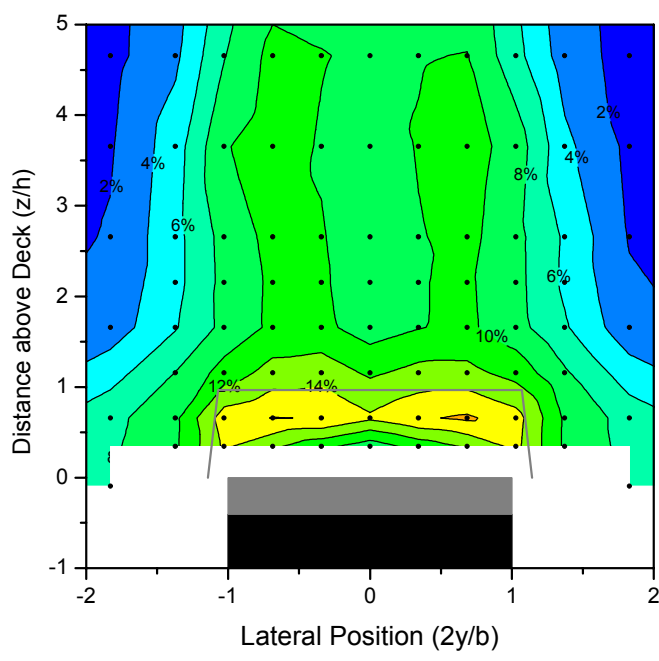

Figure 4.13. Velocity standard deviation of vented porous surface configuration $(\mathbf{0 . 0 6 2 5}$ " holes, run 3).
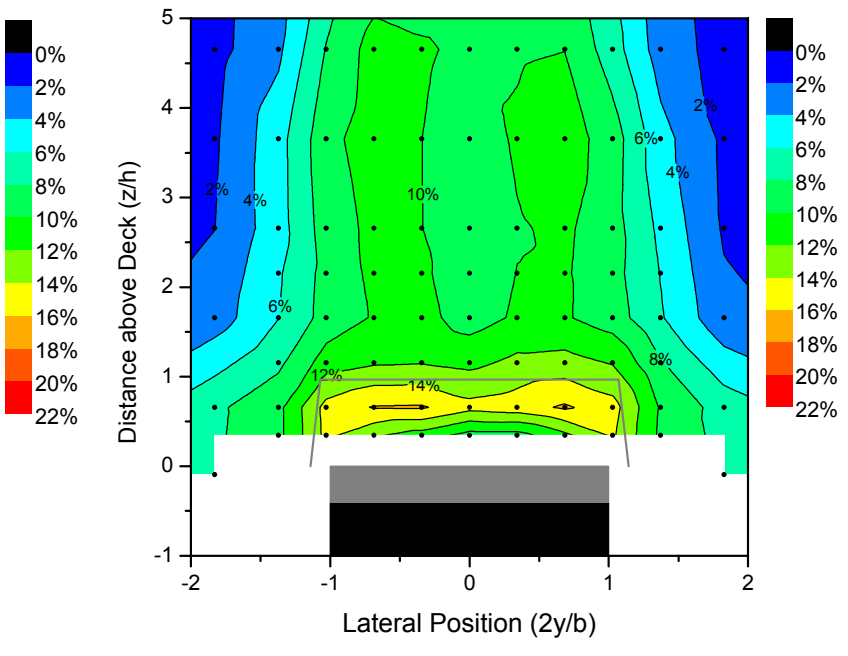

Figure 4.14. Velocity standard deviation of non-vented porous surface configuration (0.0625" holes, run 5).

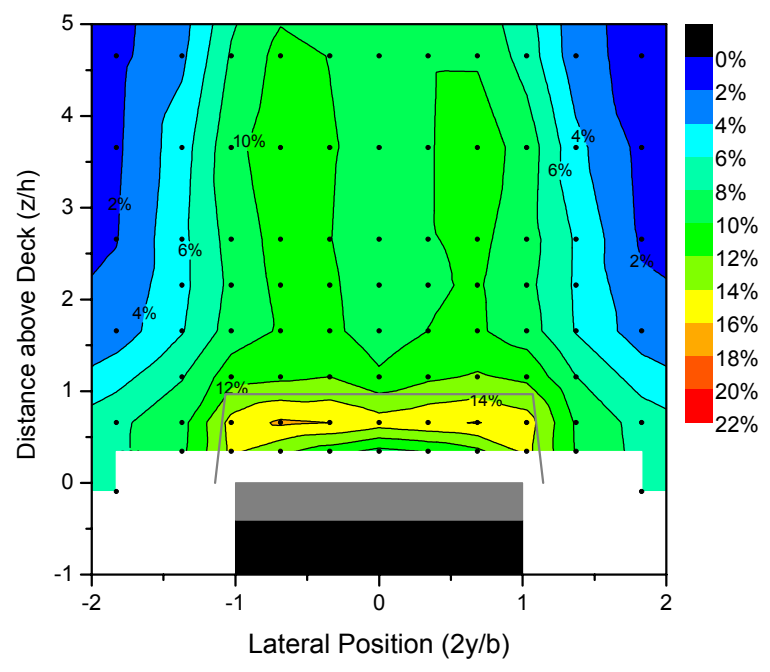

Figure 4.15. Velocity standard deviation of non-vented porous surface configuration (0.024" holes, run 6). 
The main reason for this is because if the holes were scaled up to the actual size of the ship, the smaller holes would be much more practical; a model scale of 0.024 inches scales up to 3.46 inches in full scale.

Injection was then added to the model with porous surfaces. Figure 4.16 shows run 7 , which uses the circular baffle. Mass flow was 4 cubic feet per minute which would ideally result in a uniform injection velocity of less than $1 \%$ of the freestream. However, flow visualization showed that the injection uniformity is poor. Comparing Figure 4.16 to 4.15 , this type of injection has increased the areas of maximum standard deviation. The next active technique was to inject through the porous hangar face in the freestream direction. Mass flow was set to 4 cubic feet per minute, the highest obtainable with the equipment available. By reducing the area of the surface injection, the new injection velocity is $2.5 \%$ of the freestream. Because the injected air was deflected by using a flat plate, there is still no assurance of uniform injection across the hangar face. However, flow visualization showed a reasonably smooth, symmetric distribution across the hangar face. Figure 4.17 shows the velocity standard deviation for this configuration. Unsteadiness is decreased more with this configuration than with all others. Further improvements are expected as the injection velocity is increased. Unfortunately, due to hardware limitations, higher velocities could not be tested. Finally, run 9 was conducted to determine if the improvements in run 8 could be obtained without using a porous flight deck. Figure 4.18 shows the corresponding plot. In addition to the asymmetric feature, the source of which is unknown, the flow field is more unsteady than for the prior case (run 8). As a result, it is concluded that the reduction in unsteadiness in run 8 is caused by a combination of both the porous flight deck and the injection from the hangar face. 
By analysis of the contour plots, this configuration (run 8) causes the greatest reduction in unsteadiness of all the porous surface techniques tested during this investigation.
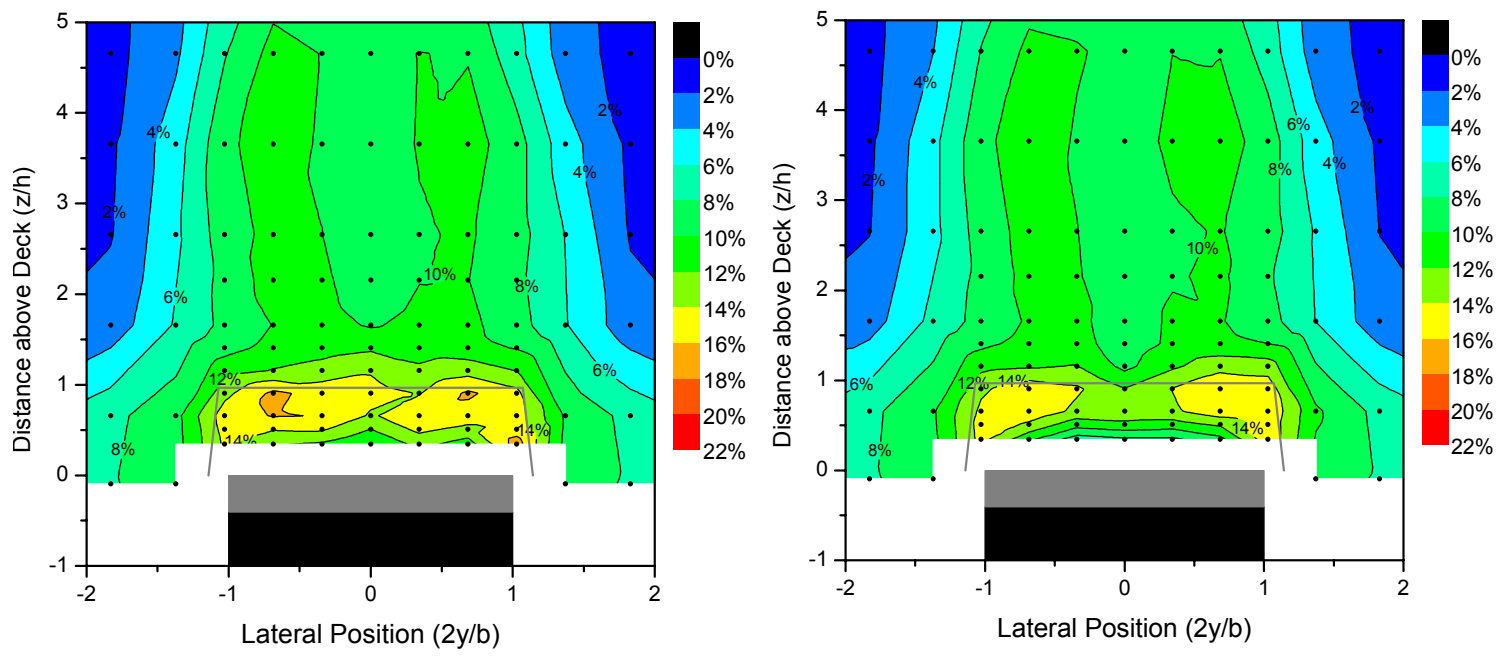

Figure 4.16. Velocity standard deviation of run 7 Figure 4.17. Velocity standard deviation of run 8 (injection using circular baffle). (injection from hangar face).

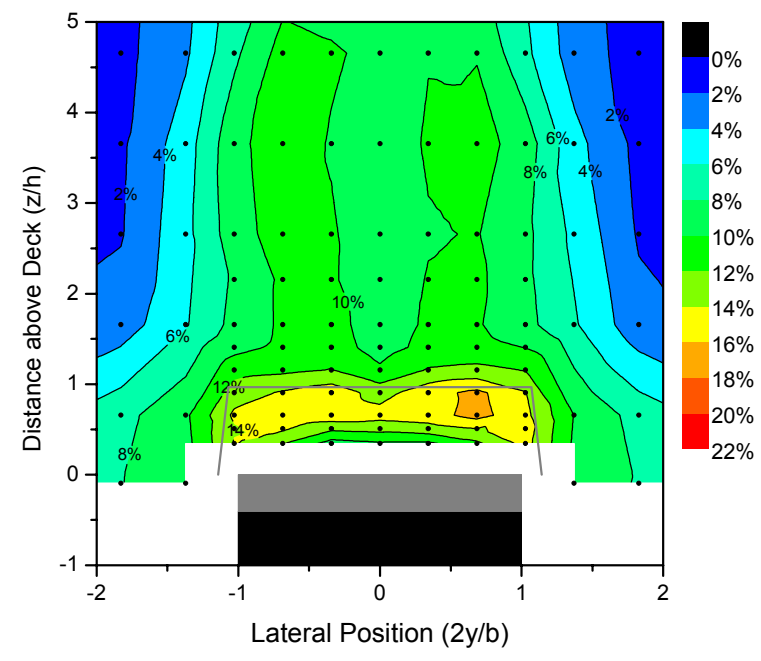

Figure 4.18. Velocity standard deviation of run 9.

The second portion of the investigation explores the effects of fence configurations. Figures 4.19 and 4.20 show the velocity standard deviation measured with a $1 / 2$ inch serrated porous fence (run 11) and a $1 / 2$ inch triangular-notched porous fence (run 12), respectively. Note that the height of the hangar is 1 inch on the model. Because of their frontal area, fences tend to push the unsteady shear layer upward and 
outward. Although this results in a region of increased unsteadiness directly downstream of the fence edge, the unsteadiness inside the edges and downstream of the hangar is significantly reduced. The serrated fence has a more turbulent shear layer, as the triangular notches do not cause peaks in unsteadiness to be as large. Both configurations, however, result in much lower unsteadiness downstream of the hangar face.

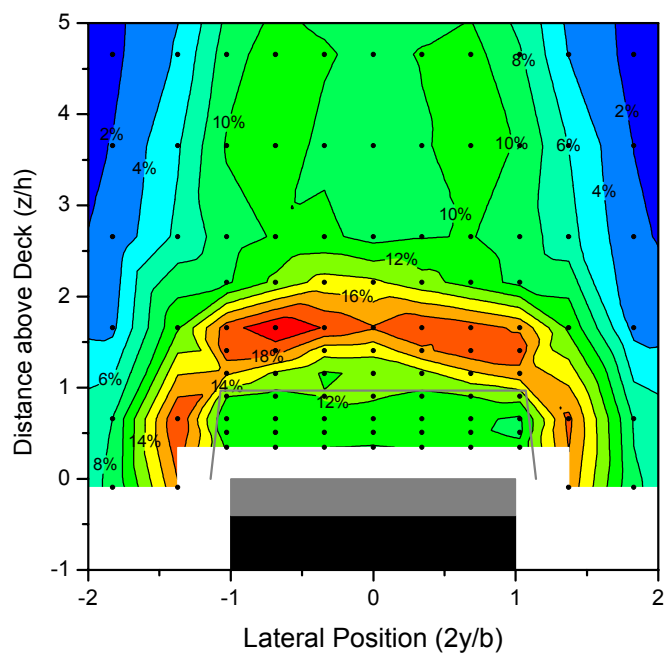

Figure 4.19. Velocity standard deviation of the serrated porous fence configuration (run 11).

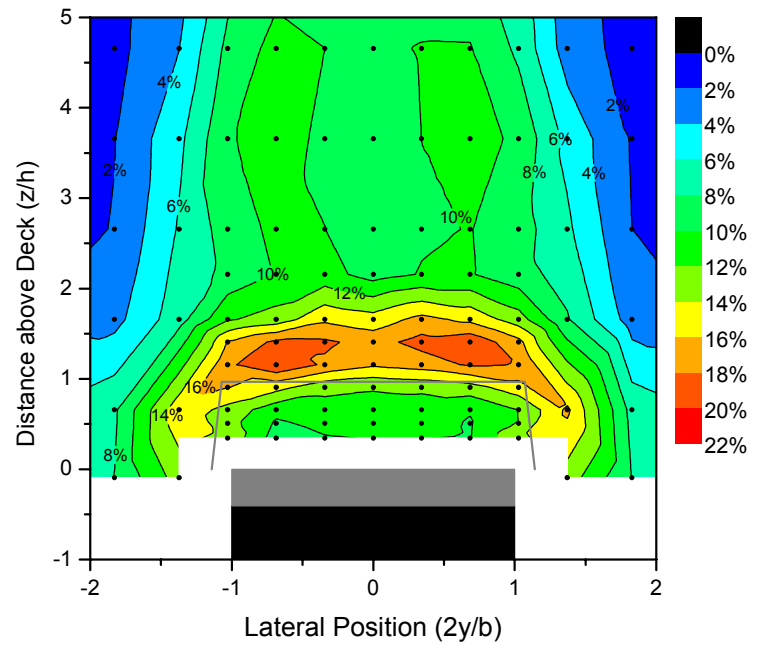

Figure 4.20. Velocity standard deviation of the triangular-notched porous fence configuration (run12).

The next two figures show the flow field effects of the "mini delta wings". Figure 4.21 shows run 13 which was constructed from porous sheet metal. The results show decreased unsteadiness downstream of the fence edge, but the device no longer shields the flight deck like the prior two fences did. Because the device was porous, a solid version was also constructed to promote better formation of the small leading-edge vortices. Figure 4.22 shows the resulting contour plot. Unfortunately, improvements were not discovered. The asymmetries of the flow field may be caused by imperfections in the fabrication process. 
The final fence configuration tested was constructed of reticulated foam. Figure 4.23 shows the contour plot. This device is superior to the other fences for two reasons.

First, it does not have as much unsteadiness downstream of the fence edge as runs 11 and 12. Most importantly, however, this configuration actually shields the area behind the hangar most effectively, resulting in the lowest amount of unsteadiness. In conclusion, the reticulated foam fence performs the best.

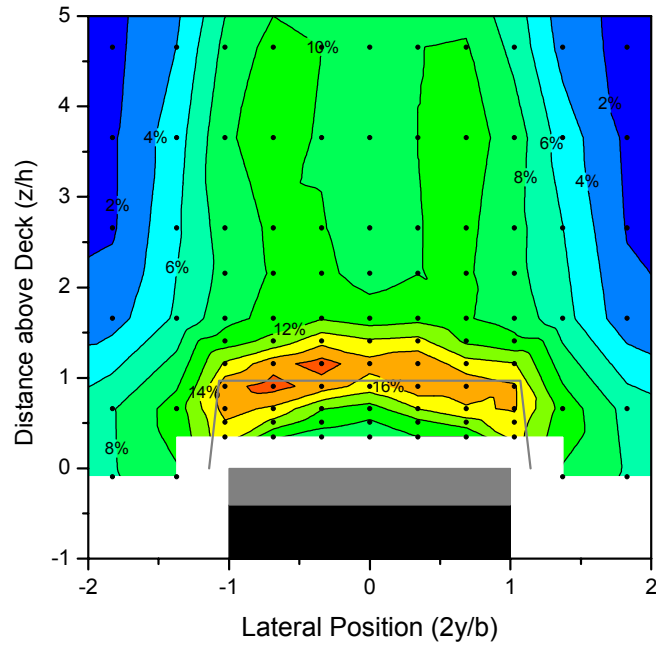

Figure 4.21. Velocity standard deviation of porous "mini delta wing" configuration (run 13).

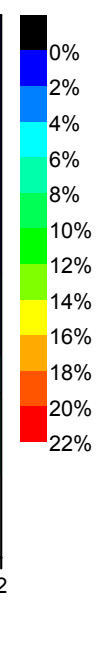

Figure 4.22. Velocity standard deviation of solid "mini delta wing" configuration (run 14).

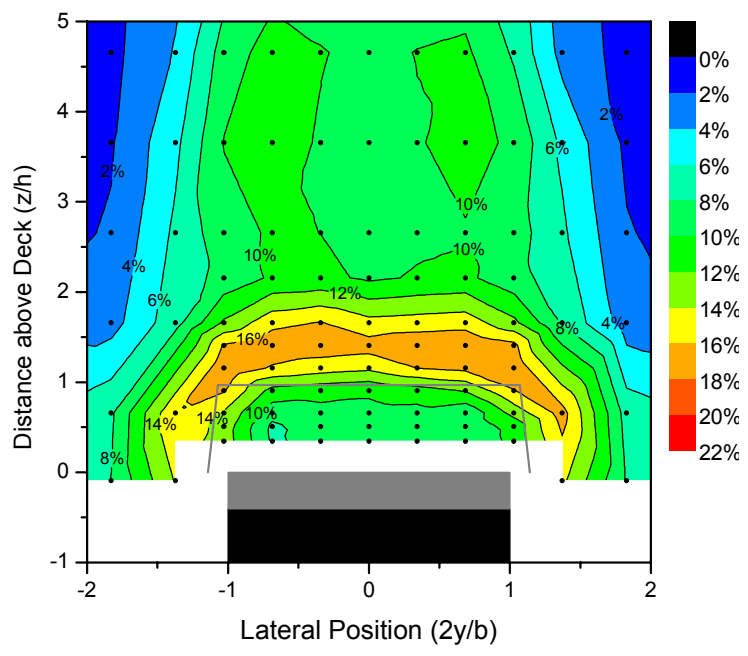

Figure 4.23. Velocity standard deviation of reticulated foam fence configuration (run 15). 


\subsubsection{Velocity standard deviation line plots}

As in the case of the mean velocity contour plots, the standard deviation contour plots can be understood further by creating line plots. Figures 4.24 and 4.25 show the standard deviation at a height corresponding to the rotor height at low hover. As mentioned before, this height is $z / h=0.66$. All of the configurations show an improvement over the flight deck $(|2 y / b|<1)$ compared to the baseline. Consistent with the other plots, the configuration tested in run 8 leads to the greatest improvement. The fence configurations produce large peaks outside the width of the ship, but allow for substantial reductions in unsteadiness behind the hangar. The reticulated foam fence (run 15) causes the greatest reduction in unsteadiness.

Figures 4.26 and 4.27 show the standard deviation along the vertical centerline of the measurement plane. The same trends are observed here. The porous surface and blowing configurations (runs 3 to 9) generally decrease unsteadiness along the entire centerline, and the fence configurations (runs 11 to 15) increase and shift the peak standard deviation further above the flight deck. 


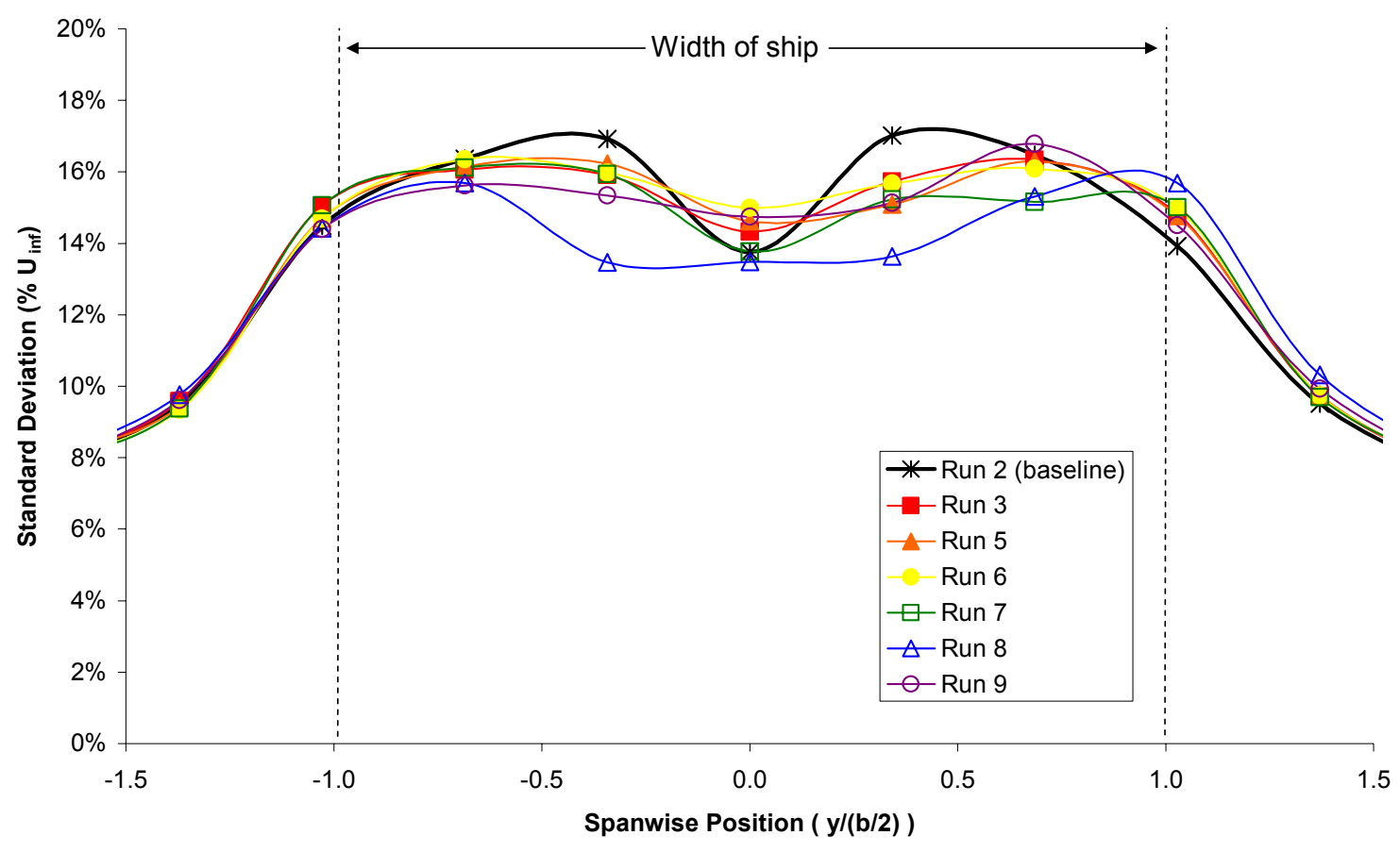

Figure 4.24. Velocity standard deviation at low hover for porous surface configurations.

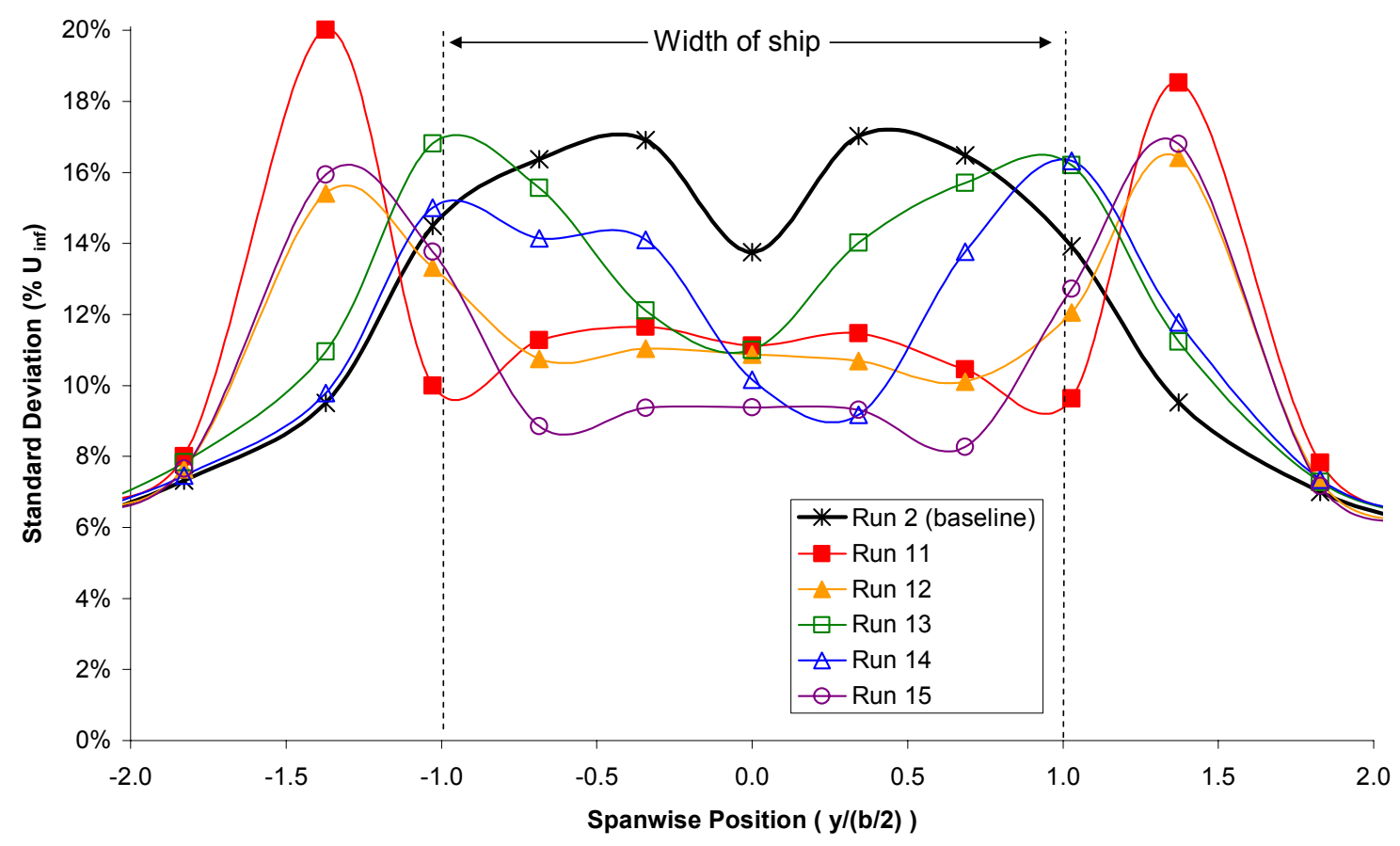

Figure 4.25. Velocity standard deviation at low hover for fence configurations. 


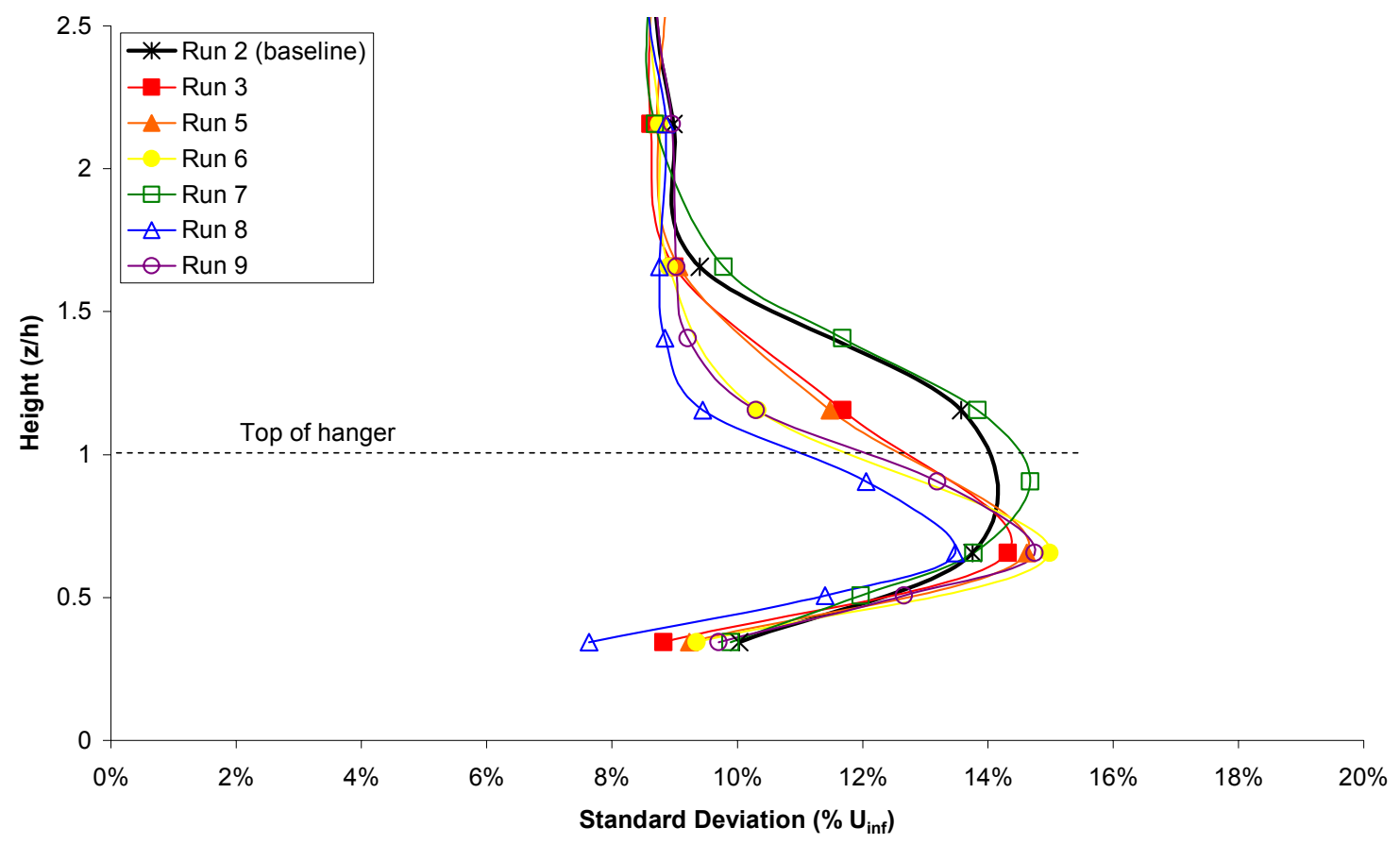

Figure 4.26. Velocity standard deviation along vertical centerline for porous surface configurations.

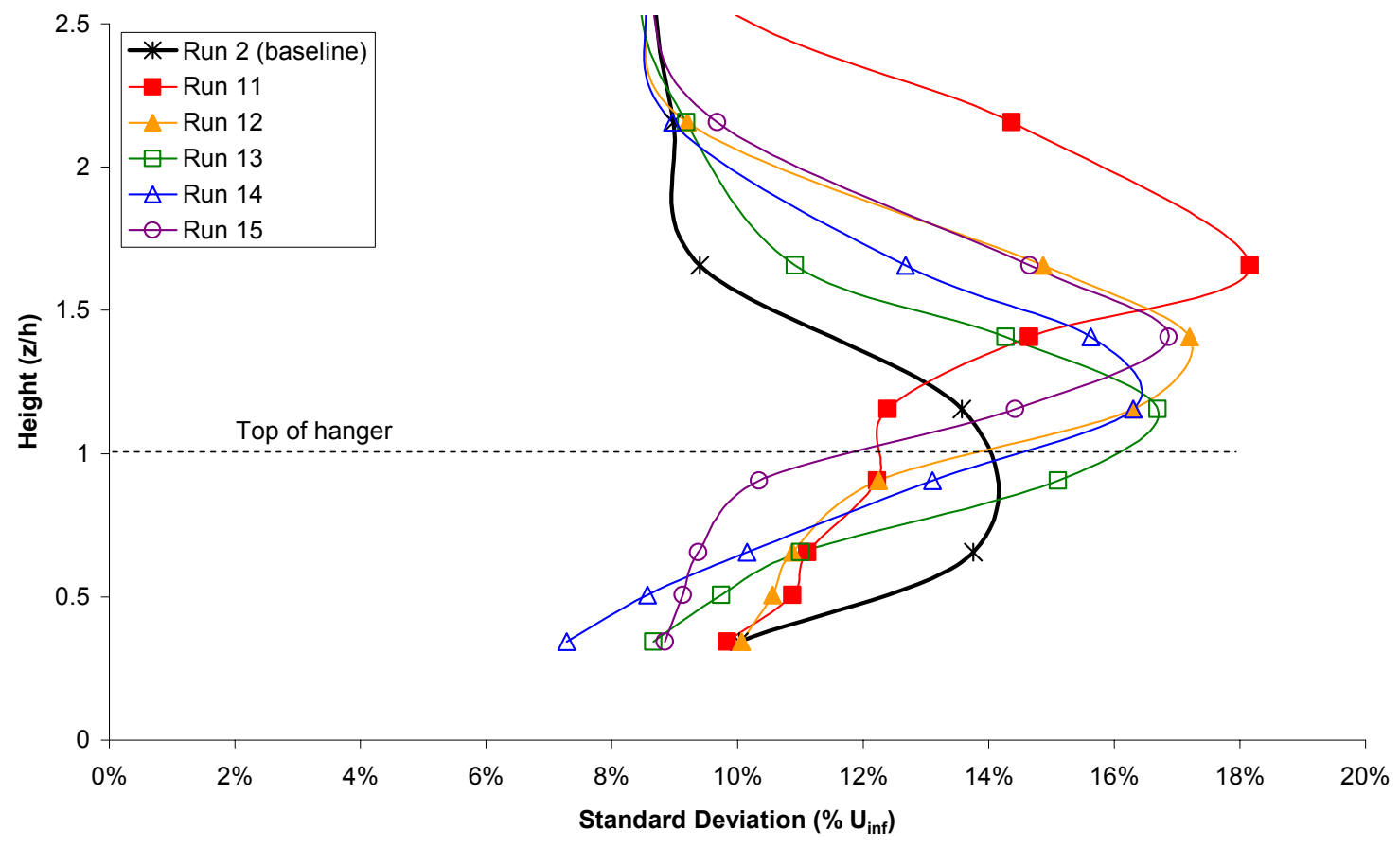

Figure 4.27. Velocity standard deviation along vertical centerline for fence configurations. 


\subsection{Turbulence intensity}

Turbulence intensity is defined as:

$$
T I=\frac{\sigma}{\overline{U_{\text {local }}}}
$$

which is the standard deviation divided by the total local mean velocity. This parameter is often important in unsteady aerodynamic investigations; however its relevance to this application is questionable. In ship airwake flow fields, the range of local velocities in the vicinity of the flight deck can vary by almost an order of magnitude. Recalling Figure 4.1 , the mean velocity decreases to the $20 \%$ range of freestream velocity behind the hangar. Because this parameter is in the denominator of the turbulence intensity calculation, TI can become large very quickly. In addition, this application typically causes the standard deviation to increase as the mean velocity decreases. As a result, it becomes difficult to determine the effect of each flow control device. The following discussion shows the difficulty in evaluating the flow control devices with this parameter alone. Recall from the uncertainty analysis that regions of high turbulence intensity (above 20\% measured, typically below one hangar height) should be analyzed qualitatively only.

\subsubsection{Turbulence intensity contour plots}

Figure 4.28 shows the turbulence intensity for the baseline configuration. Again, all the plots in this section have the same contour levels and colors, ranging from $0 \%$ (blue) to $65 \%$ (red) turbulence intensity. The baseline configuration has a radial contour pattern and a maximum intensity of 50 to $55 \%$. By comparing this figure with the 
baseline mean velocity and standard deviation contours (Figures 4.1 and 4.12), one can see that the shape of the turbulence intensity is driven by the mean velocity, not the standard deviation. It is for this reason that turbulence intensity is not a robust parameter for evaluation of flow control devices. Figures 4.29 and 4.30 show contours for the porous surface configurations. The first presents the results from run 3 and is almost identical to all the passive porous surface configurations. Figure 4.30 shows slightly higher maximum turbulence intensities for run 8 when compared with the other porous surface configurations. Like the baseline, however, these contours are shaped from the mean velocity. The differences between the configurations shown by the standard deviation are simply not visible here.

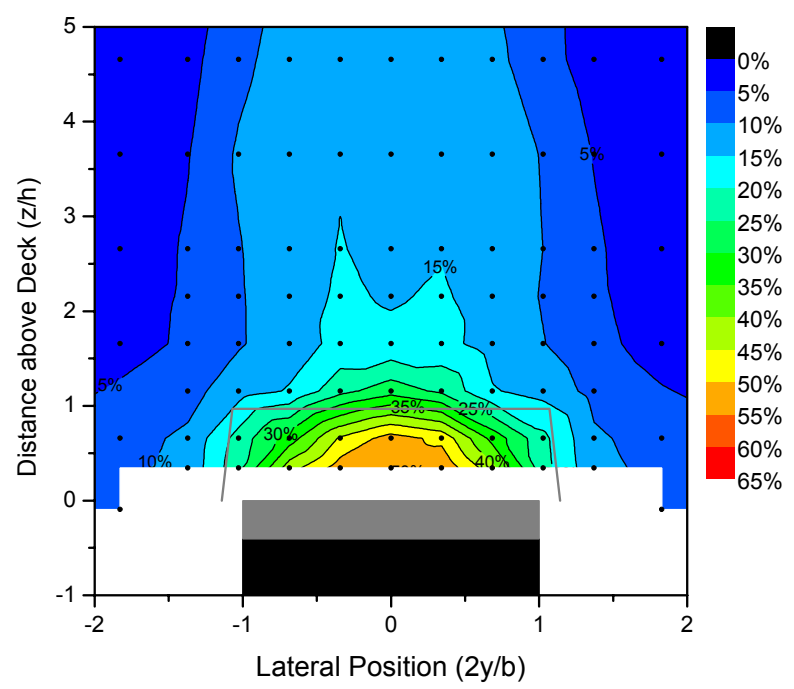

Figure 4.28. Turbulence intensity of the baseline configuration. 


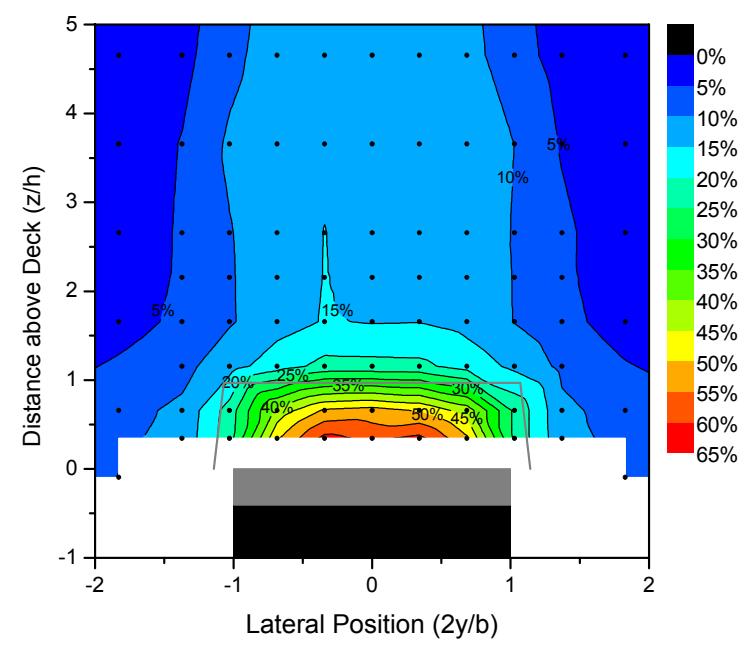

Figure 4.29. Turbulence intensity of run 3.

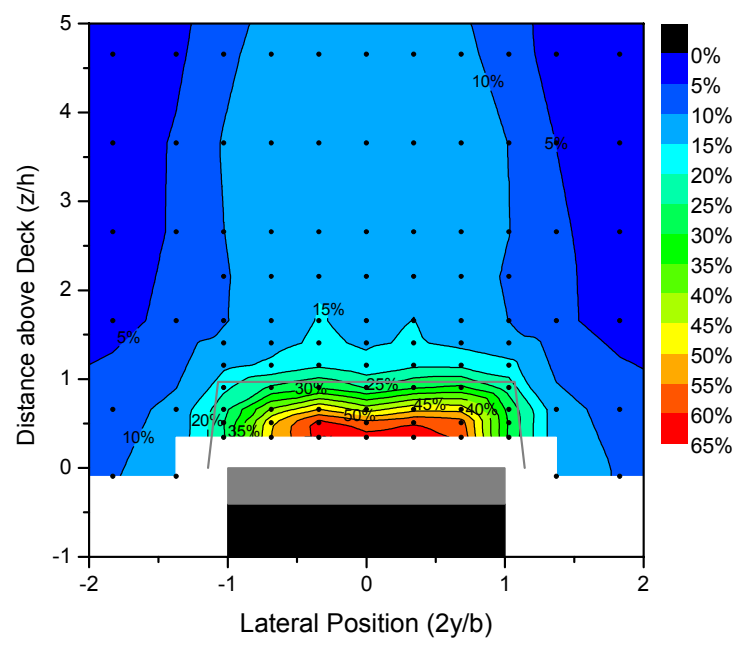

Figure 4.30. Turbulence intensity of run 8.

Unlike the porous surface configurations, it has been shown that fences tend to have a greater impact on the flow. Figure 4.31 shows the turbulence intensity contours of the serrated porous fence configuration (run 11). By comparing the mean velocity and standard deviation contours (Figures 4.4 and 4.19), one notices that the mean velocity is no longer driving the shape of the contours. Instead, the turbulence intensity now appears as the combination of the two variables which define it. Run 11 shows a significant reduction in the turbulence intensity near the flight deck when compared with the baseline. However, this plot fails to show the severity and location of the shear layer formed at the top of the fence as shown in the standard deviation plot. Figures 4.32 to 4.34 show similar results for other fence configurations. An interesting feature is that the lowest turbulence intensity near the flight deck occurs with the device which has the largest frontal area, and the opposite occurs for the device with the smallest frontal area. This implies that devices with large frontal areas (the serrated porous fence, for example) are superior; however the standard deviation metric showed that this configuration 
produced the largest peaks in unsteadiness. Thus, the turbulence intensity metric appears to be unable to evaluate control devices accurately.

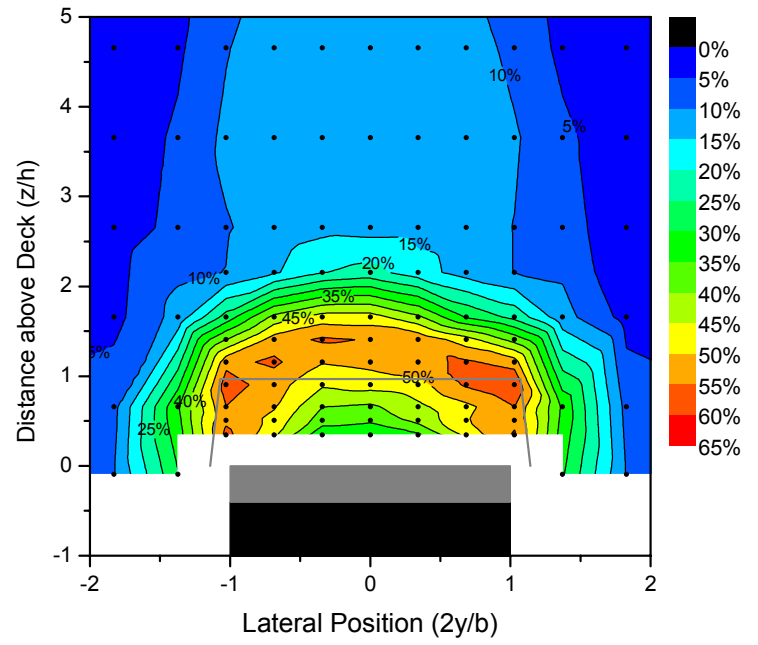

Figure 4.31. Turbulence intensity of run 11.

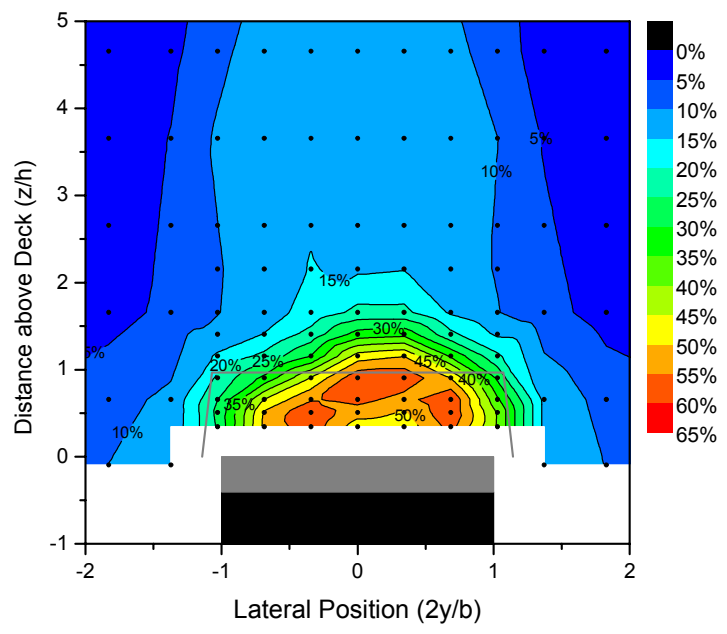

Figure 4.33. Turbulence intensity of run 14.

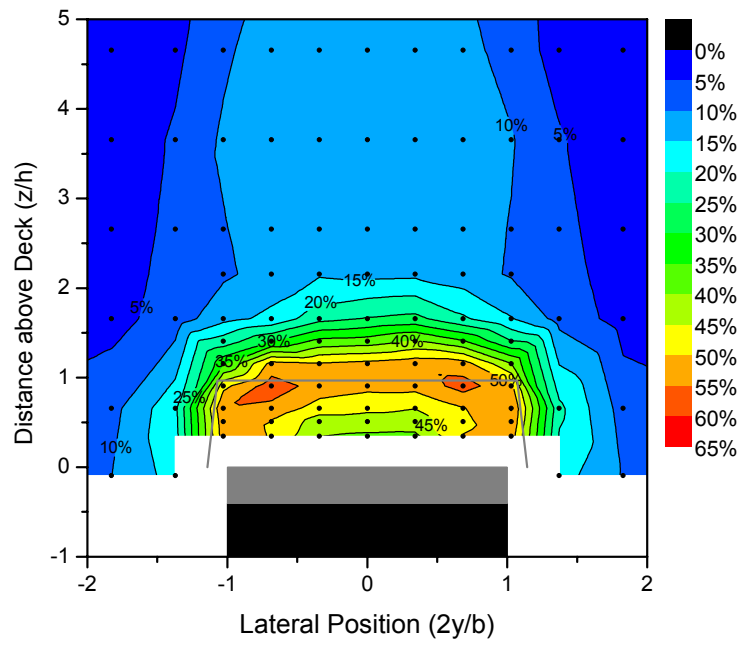

Figure 4.32. Turbulence intensity of run 12 .

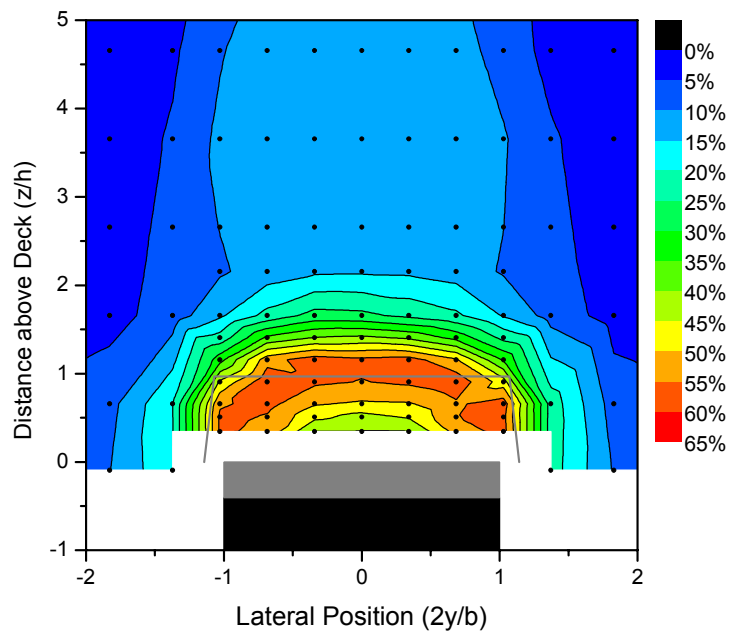

Figure 4.34. Turbulence intensity of run 15 .

\subsubsection{Turbulence intensity line plots}

Line plots of the turbulence intensity enable one to quantify the statements made in the prior section. Figures 4.35 and 4.36 show the turbulence intensities corresponding to a low hover height $(z / h=0.66)$. By visual inspection, it can be seen that for every configuration, the average turbulence intensity across the flight deck is higher than the 
baseline. If this were the only parameter analyzed, one would falsely conclude that none of the flow control devices offer improvement. Figures 4.37 and 4.38 show the turbulence intensity along the vertical centerline $(2 y / b=0)$. All of the porous surface configurations (runs 3 to 9) show an increase in intensity very near the deck, followed by a steady reduction until $z / h=1.5$. Run 8 , the configuration with porous surfaces and injection from the hangar face, yields the greatest reduction. The fence configurations (runs 11 to 15 ) have the opposite effect. Figure 4.38 shows that the turbulence intensity is decreased near the flight deck, but increases significantly near the top of the hangar and above. Inspection shows that run 11 may be the best configuration. However, as shown in the prior two sections, the reticulated foam fence (run 18) seems to be the better performer, chosen for its low standard deviation and mean flow near the flight deck. Because these two features are desirable, the ratio of the two statistics (the definition of turbulence intensity) makes it impossible to draw conclusions about the devices. As a result, turbulence intensity is not a good evaluation parameter and should not be used exclusively. 


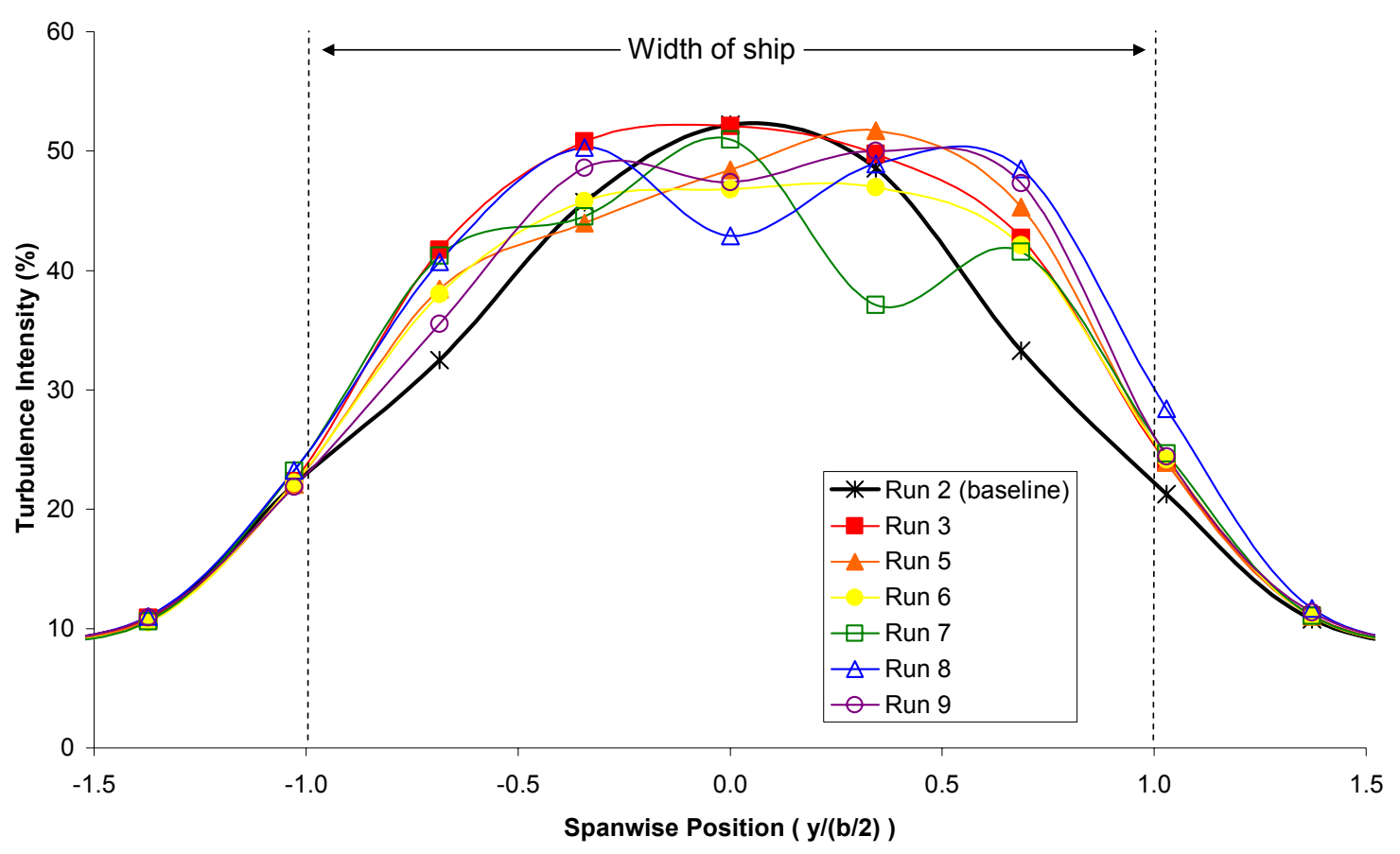

Figure 4.35. Turbulence intensity at low hover $(z / h=0.66)$ for porous surface configurations.

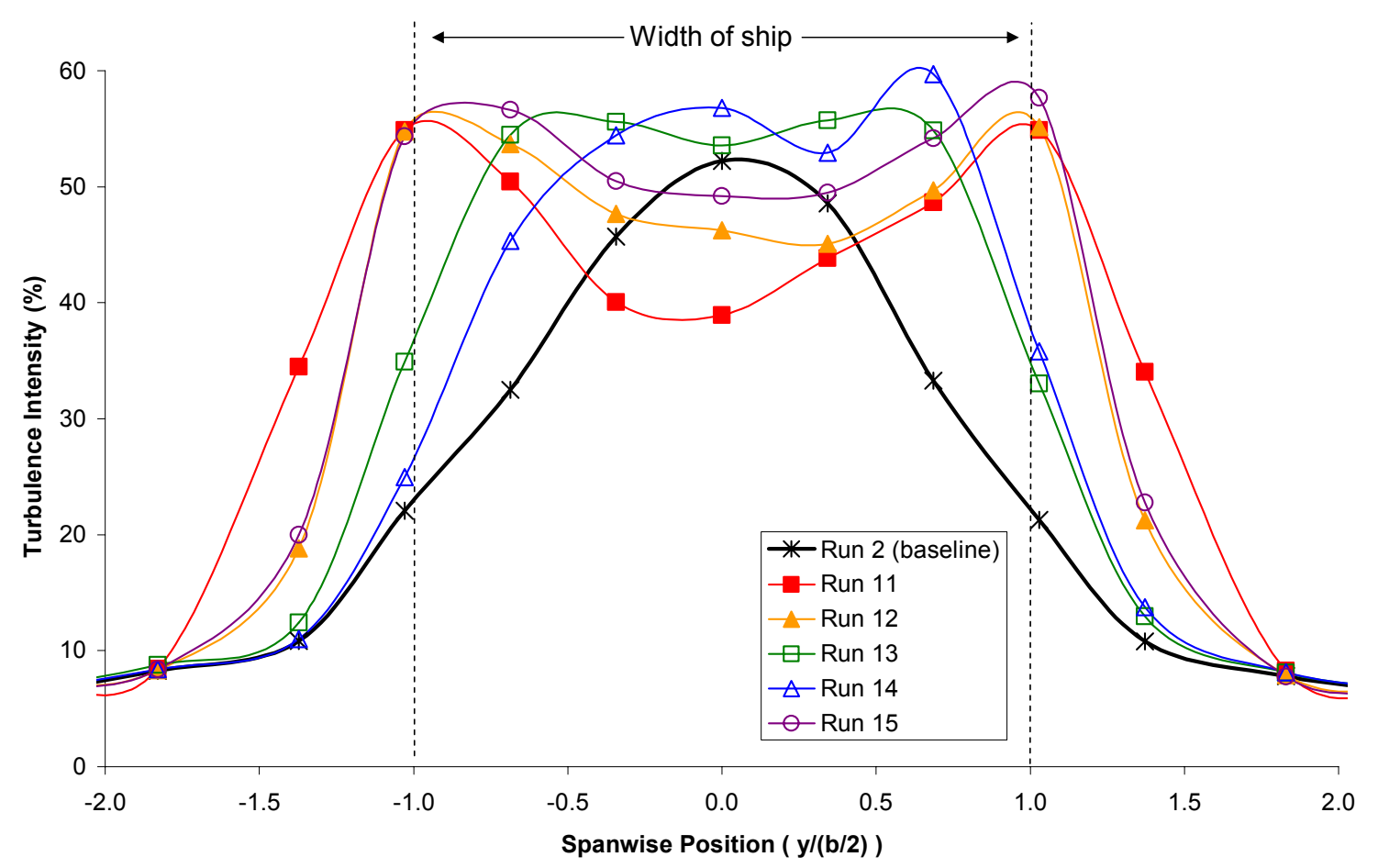

Figure 4.36. Turbulence intensity at low hover $(z / h=0.66)$ for fence configurations. 


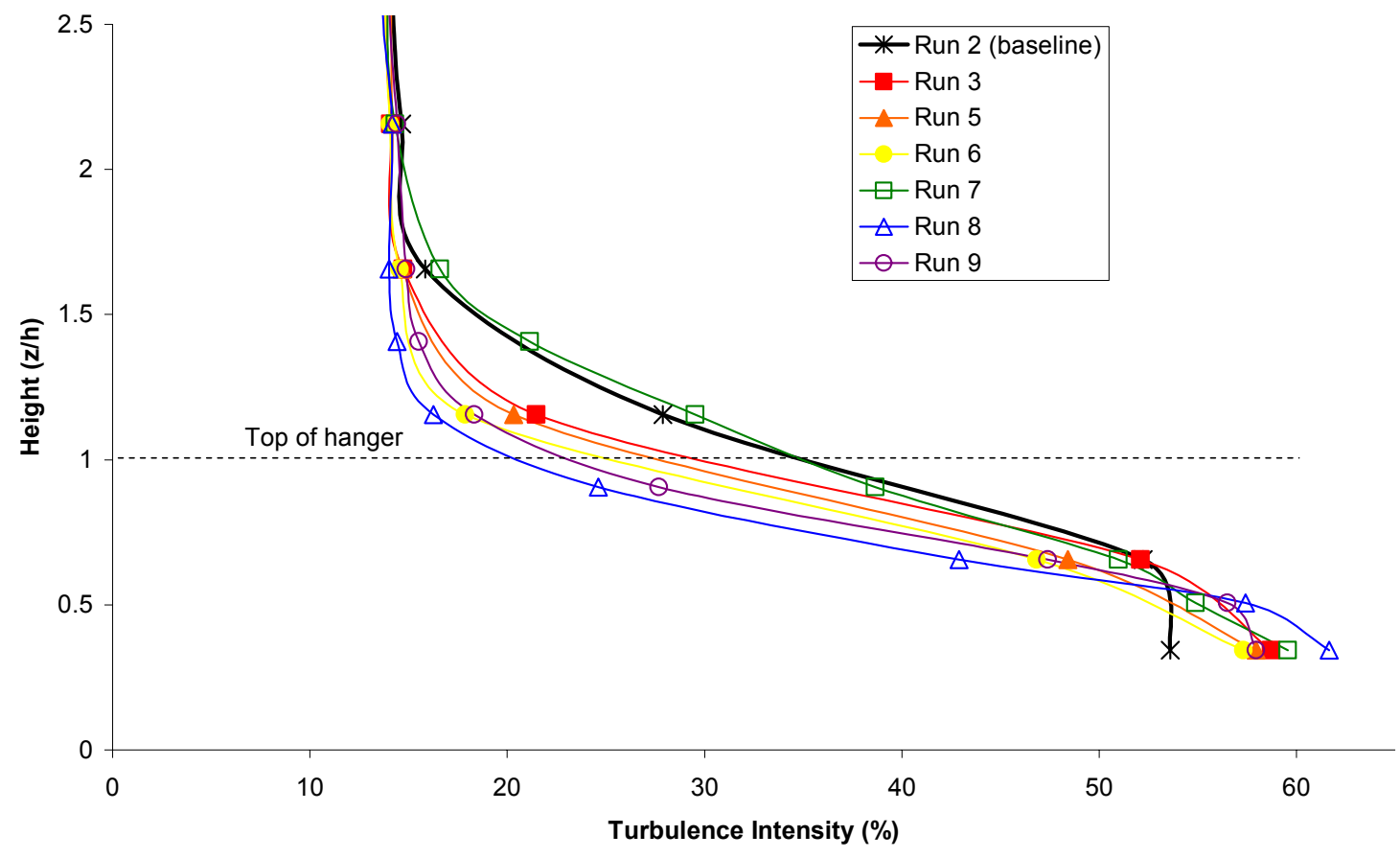

Figure 4.37. Turbulence intensity on the vertical centerline $(2 y / b=0)$ for porous surface configurations.

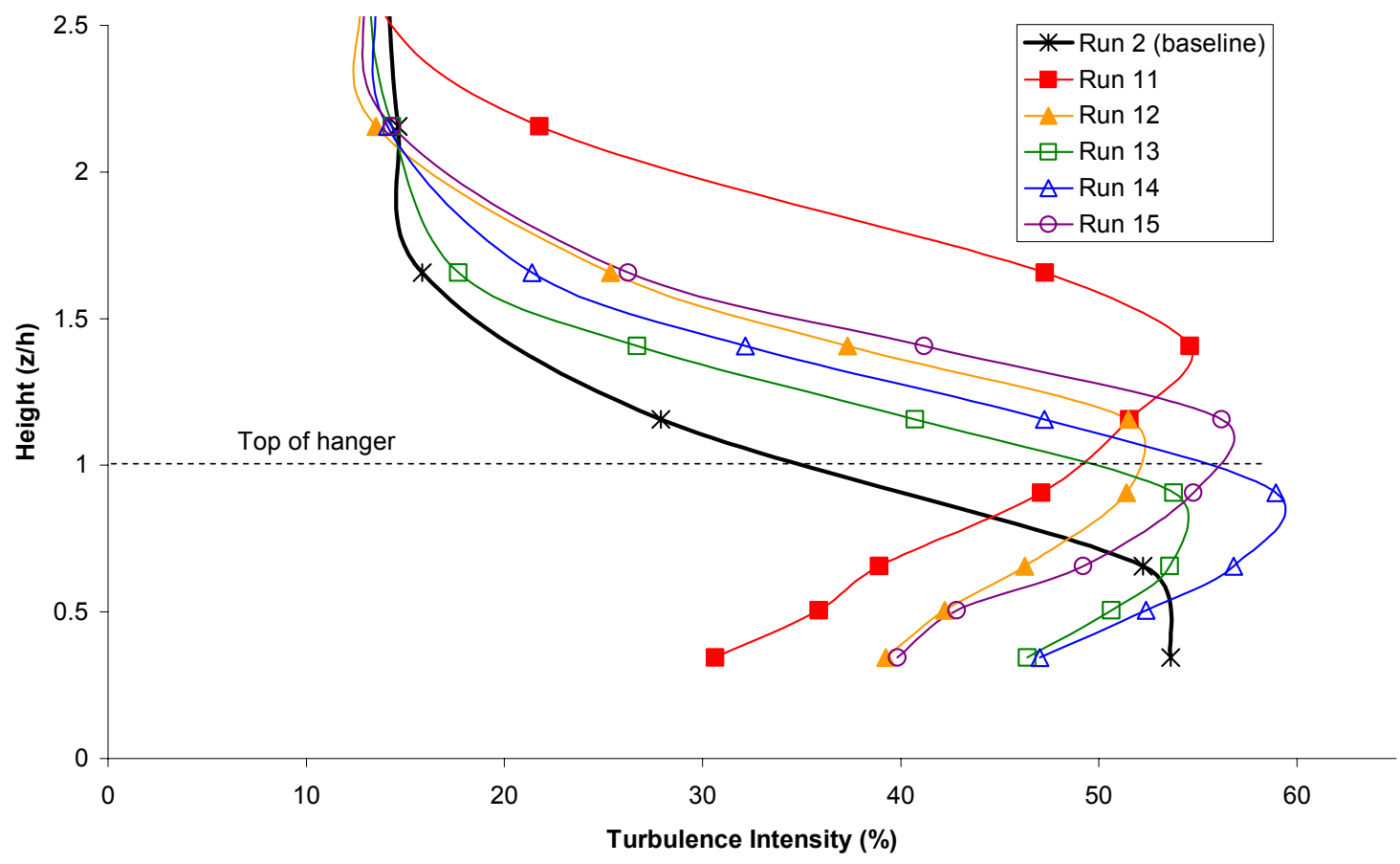

Figure 4.38. Turbulence intensity on the vertical centerline $(2 y / b=0)$ for fence configurations. 


\subsection{Average unsteadiness in landing area}

The prior sections in this chapter have concluded that the porous surface configuration with injection from the hangar face (run 8) and the reticulated foam fence (run 15) appear to be the superior techniques for reducing unsteadiness. It has been shown that each offer their own advantages and disadvantages, but it is difficult to determine which are important to a pilot. Consequently, the following metric is considered.

Helicopter approaches to ships are flown on a specific path. For DDG class ships, helicopters approach directly from the stern. Most pilots then come to a stop relative to the ship and hover about 10 feet over the landing spot before descending to the deck. ${ }^{37}$ By considering this altitude and the height of a typical naval helicopter, the altitude of the highest point on the helicopter can be calculated. Consequently, it is assumed that the airwake above this height does not affect the handling qualities of the helicopter. This is a key assumption, and the method with which this height is chosen is important. To be the most accurate, this height should be 1 to 2 rotor diameters above rotor height. However, because others ${ }^{23}$ have shown that the unsteady fuselage loads can be correlated with pilot workload, the airwake entrained by the rotor may be of lesser importance. Therefore a lower height is acceptable, and it is first assumed to be a few feet above the rotor. Similarly, spanwise limits can be placed on the airwake, outside which it is assumed that there are again no effects on the helicopter. These limits are initially chosen at the edges of the ship. Figure 4.39 shows how these constraints appear on the baseline velocity standard deviation flow field. The upper limit is at $z / h=1.9$. 


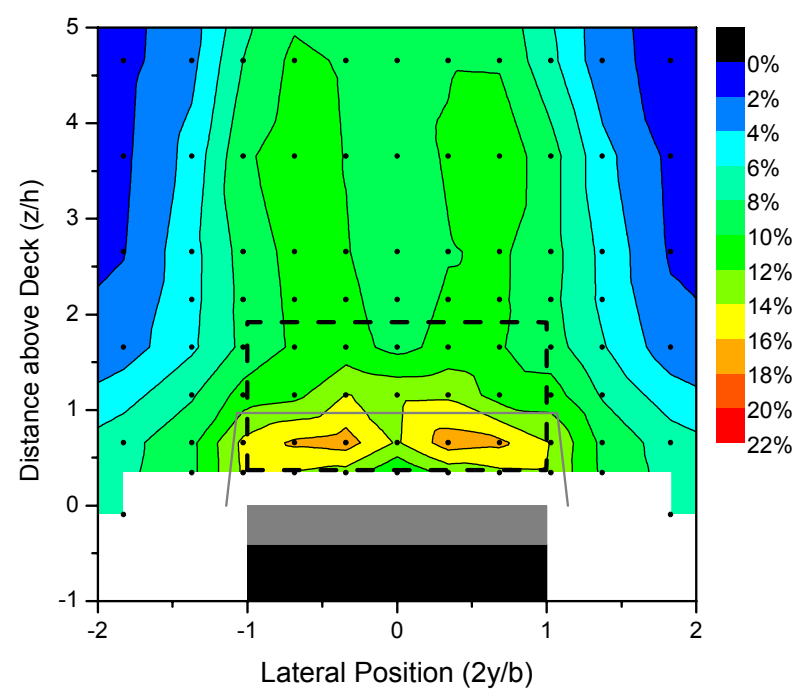

Figure 4.39. Baseline velocity standard deviation. The box encloses the region in the airwake which affects helicopter operations.

With a fixed landing region in the flow field defined, the average unsteadiness can be calculated by computing the mean of the standard deviation at every grid point in this region. This is analogous to computing the volume under a three-dimensional contour plot and dividing by the area of the data plane. Alternatively, calculating this average using the variance instead of the standard deviation would provide a measurement of the total kinetic energy in the flow in the landing region. However, because results presented thus far have been based on standard deviation, the average unsteadiness metric is calculated using the standard deviation. Noting that the experimental grid points in this region are not equally spaced, a uniformly spaced grid must first be produced. Failing to do so would result in weighting the values of closely spaced points more heavily. The same linear interpolation used to create the contour plots is used to create a 900 point, equally spaced grid. Finally, the values are averaged, resulting in a single number which can be used for comparison between configurations. 
Table 4.1 lists each configuration with the average unsteadiness parameter and the corresponding percent change from the baseline configuration. Figure 4.40 shows a visual comparison. The previous conclusions for the porous surface configurations (runs 3 to 9) are consistent with this new metric, and the improvements have now been quantified with a single parameter. By using a porous flight deck and injection from the hangar face (run 8 ), the unsteadiness is reduced $6.56 \%$. The fence configurations, however, all reveal an increase in unsteadiness. All of the fences tested had a height of $0.5 \mathrm{~h}$, which does not push the shear layer out of the landing region defined above. Although the unsteadiness was significantly reduced behind the hangar, the current metric also factors in the unsteadiness of this shear layer (occurring at a height around $z / h$ $=1.5$ ). If the fence were two or three times higher, the shear layer would then be outside of the defined landing region, and the average unsteadiness parameter would decrease. By comparing runs 11 to 15 with each other, additional observations can be made. The serrated porous fence (run 11) and the triangular-notched porous fence (run 12) cause a considerable increase in unsteadiness in the landing region (14.99\% and $13.04 \%$ respectively). As a result, these configurations may not be the best option even if the size of the fence, and hence the "sheltered" region, is increased. The remaining configurations do not cause as significant increases in this investigation, so they should perform better if their height is increased. Relative to the amount of frontal area of the remaining fences, the reticulated foam fence (run 15) produces the least amount of additional unsteadiness in the landing region. Therefore, it is recommended that future investigations include a large (on the order of the height of the hangar) reticulated foam fence. 
Table 4.1. Average unsteadiness of each configuration.

\begin{tabular}{|c|c|c|c|}
\hline Run \# & Configuration & $\begin{array}{c}\text { Average } \\
\text { unsteadiness } \\
\text { (standard } \\
\text { deviation in \% } \\
\text { freestream) }\end{array}$ & $\begin{array}{c}\text { Percent } \\
\text { change } \\
(\%)\end{array}$ \\
\hline 2 & Baseline & 12.49 & - \\
\hline 3 & Porous surfaces $(0.0625$ " holes $)$ with "venting" & 12.20 & -2.33 \\
\hline 5 & Porous surfaces (0.0625" holes) & 12.08 & -3.27 \\
\hline 6 & Porous surfaces $(0.024 ”$ holes $)$ & 11.98 & -4.07 \\
\hline 7 & Porous surfaces $(0.024$ " holes $)+$ injection using circular baffle & 12.48 & -0.06 \\
\hline 8 & Porous surfaces (0.024" holes) + injection from hangar face & 11.67 & -6.56 \\
\hline 9 & $\begin{array}{l}\text { Porous surfaces }(0.024 \text { " holes })+\text { injection from hangar face } \\
\text { (solid flight deck) }\end{array}$ & 12.04 & -3.66 \\
\hline 11 & Serrated porous fence & 14.37 & 14.99 \\
\hline 12 & Triangular-notched porous fence & 14.12 & 13.04 \\
\hline 13 & Triangular-notched porous fence angled 60 degrees upstream & 13.48 & 7.94 \\
\hline 14 & Triangular-notched solid fence angled 60 degrees upstream & 13.12 & 5.03 \\
\hline 15 & Reticulated foam fence & 13.14 & 5.18 \\
\hline
\end{tabular}

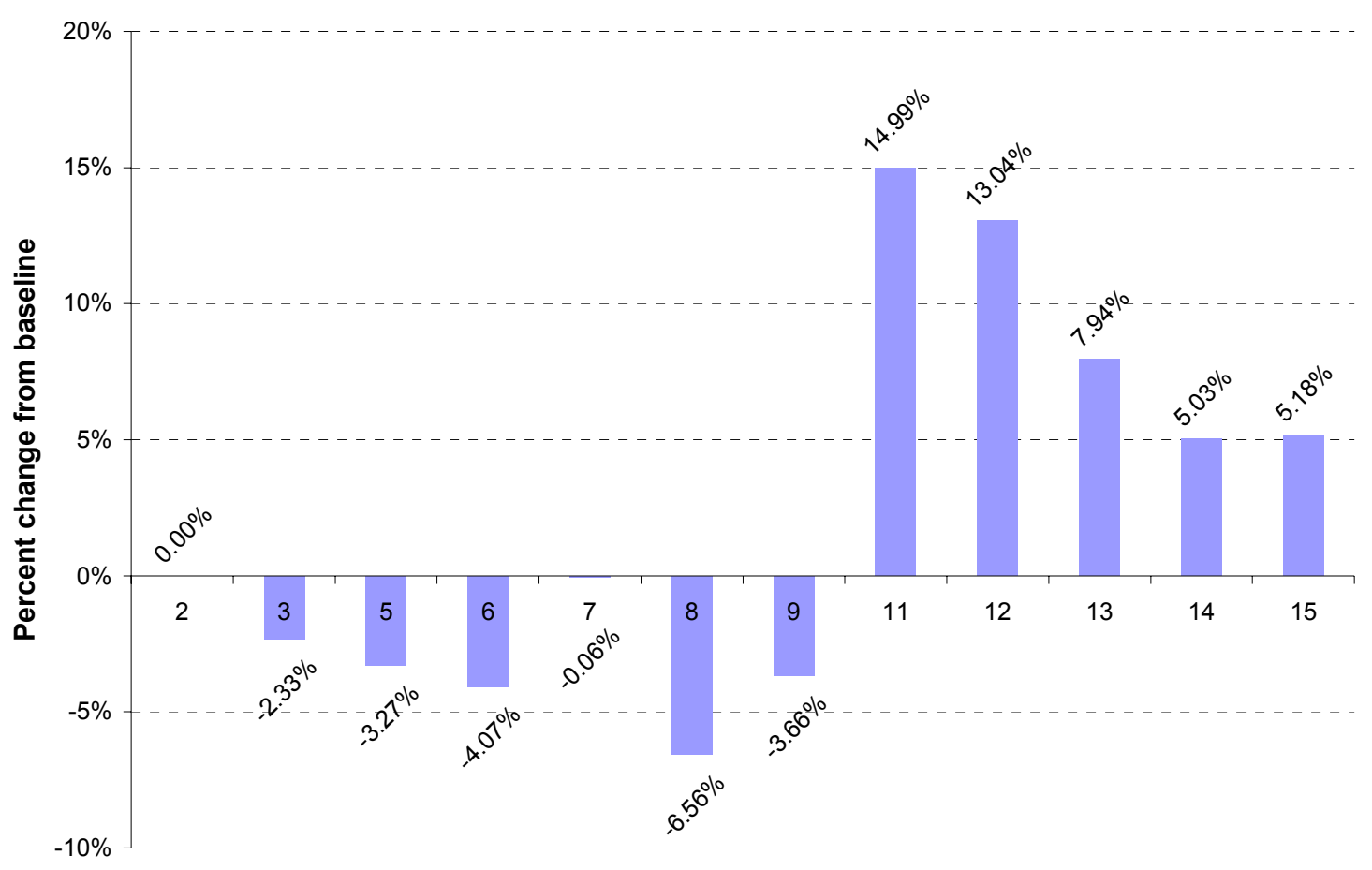

Run number

Figure 4.40. Percent change of the average unsteadiness in the landing region with respect to the baseline. 
As mentioned previously, the landing region was defined using some specific assumptions. As a result, it is of interest to determine the sensitivity of the landing region dimensions. Because it may be difficult for a pilot to remain directly over the flight deck while fighting the unsteady airwake, the landing region is expanded laterally. Figure 4.41 shows the two additional dimensions on the baseline flow field. The width has been increased to $|2 y / b|=1.4$ and $|2 y / b|=1$.7. Similarly, Figure 4.42 shows changes to upper limit of the landing region $(z / h=1.9,2.4$, and 2.9$)$. The average unsteadiness is calculated again for each configuration and landing region. Increasing the width (Figure 4.43) causes different effects for the two categories of flow control techniques. A wider landing region causes the average unsteadiness for the porous surface configurations (runs 3 to 9) to approach the baseline value (or $0 \%$ change on the chart). This occurs because these configurations do not have any protrusions in the flow, and more portions of the unaffected airwake are included in the average. On the other hand, fences have the opposite effect since the outward shifted shear layer, caused by the fences on the sides of the ship, is included when widening the landing region.

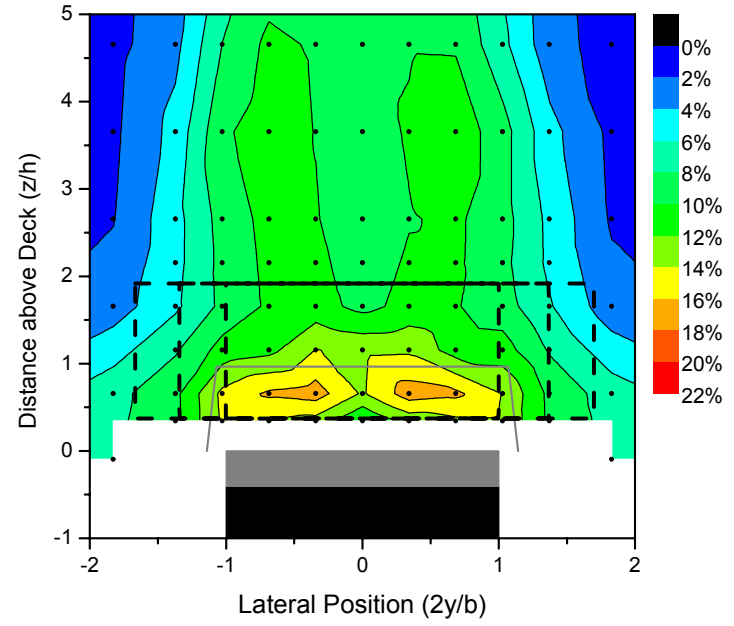

Figure 4.41. Width changes to the landing region $(|2 y / b|=1,1.4,1.7)$ overlaid on the velocity standard deviation contour plot.

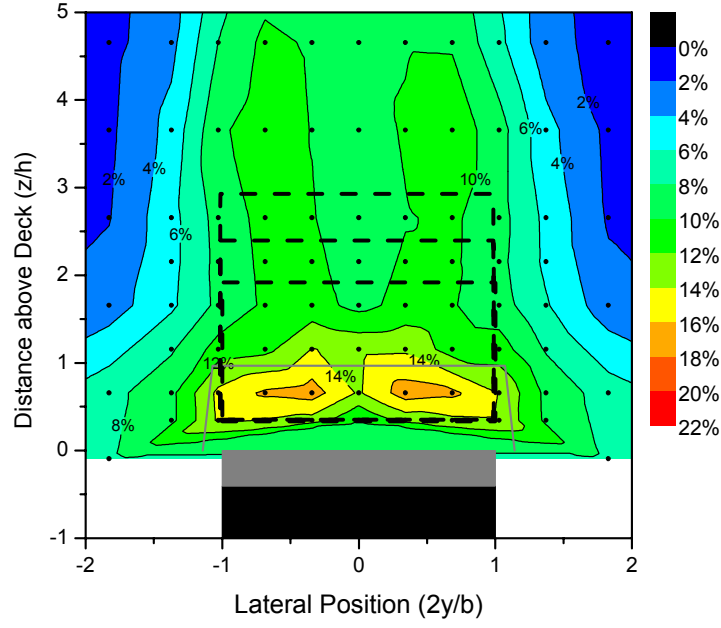

Figure 4.42. Height changes to the landing region $(z / h=1.9,2.4,2.9)$ overlaid on the velocity standard deviation contour plot. 
Increasing the height of the landing region causes a similar behavior for the porous surface configurations (Figure 4.44). Alternatively, because the top parts of the fences were already included in the original landing region, these configurations (runs 11 to 15) decrease the effect on the airwake with height. The average unsteadiness again begins to approach the baseline value. If a taller fence were used, one which pushes the shear layer completely above the defined landing region, a decrease in the average unsteadiness in this region would certainly occur. To achieve this requires a full-scale fence at least 12 feet tall. A fence on this scale could be deployable so that it is raised during helicopter/ship operations only.

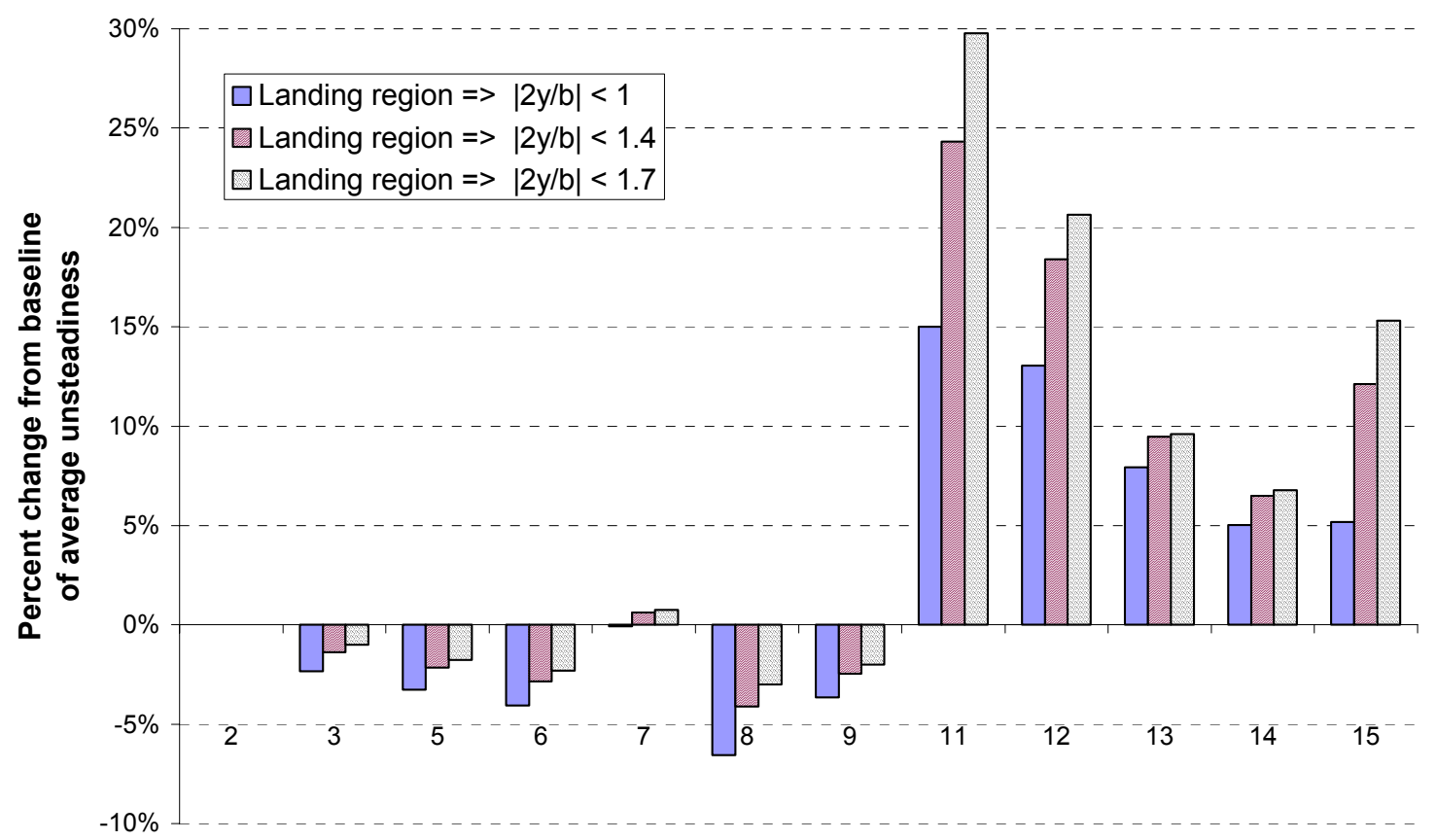

Run number

Figure 4.43. Bar graph showing the effect of widening the landing area. 


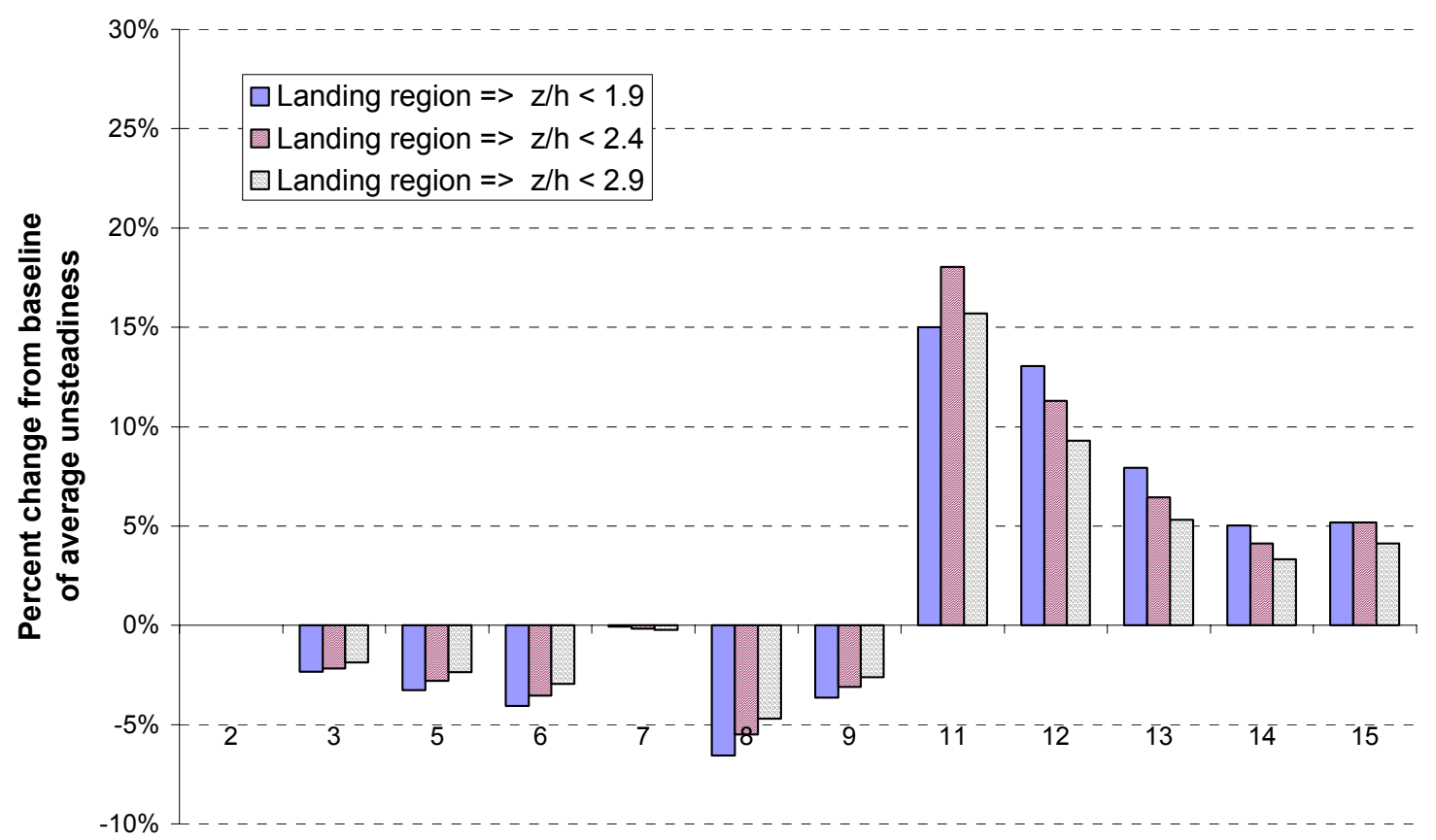

Run number

Figure 4.44. Bar graph showing the effect of raising the height of landing area.

In summary, the average unsteadiness metric is useful because different flow control devices can be compared quantitatively by a single parameter. One disadvantage of the metric is that the flow outside the landing region is completely ignored. As a result, a very large fence that completely shields the landing region is a solution. Optimizing a device for this metric alone however, could lead to uncontrollable handling qualities if a pilot were to stray out of the landing region.

\subsection{Frequency analysis}

Frequency analysis is used in fluid dynamics to identify evidence of periodic shedding. If coherent structures can be identified in the flow, new flow control techniques may be developed. Low speed flow over bluff bodies typically produces a von Karman vortex street in the wake. This type of periodicity has also been observed in 
flow visualization experiments on small naval vessels. ${ }^{11}$ Although characteristic shedding can perform significantly differently at full scale, a spectral analysis provides useful information about the behavior of the flow.

\subsubsection{Calculation of frequency spectra}

Generally speaking, frequency spectra are determined by calculating the discrete Fourier transform (DFT) of each velocity time history. Recall that the sampling rate and length is 4000 hertz for 4 seconds. As a result, information at frequencies up to 2000 hertz can be obtained without concerns of aliasing. On the other hand, the lowest frequency that can be captured depends on the amount of data acquired. Since data is collected over a four second interval, it can be assumed that frequencies as low as 0.25 hertz are resolved. This leads to a very noisy frequency spectrum from which it would be difficult to extract useful information. One would need many samples of this length to gain statistical confidence. To work around this problem, two approaches can be taken. First, many shorter duration DFTs could be averaged together at the expense of low frequency resolution, and second, the DFT could be passed through a low-pass digital filter to remove noise. This investigation used both of these techniques. Although the first is the preferred method, only a single 4-second time history was taken at each grid point. To produce many DFTs to average together, the time history must be divided into smaller segments. The consequence of this technique is a loss in frequency resolution. As a result, the number of divisions of the 4-second time history is limited so that the loss in resolution is minimized. However, the resulting DFT still contains more noise than desired; therefore it was passed through a low-pass digital filter. Details about these 
digital signal processing techniques are found in the literature. ${ }^{38}$ The MATLAB script found in Appendix D was used to calculate the DFT and plot the power spectral density (PSD). The y-axis in the PSD was scaled by the sampling frequency.

Each 4-second time history consists of 16,384 points. Prior to calculating a PSD, the mean is subtracted from the time history so that the transform occurs only on the unsteady fluctuations. The signal was then divided into 16 segments, each 1,024 points in length. A PSD is calculated for each segment, and all are averaged together. This results in a frequency resolution of approximately 4 hertz. In addition to the 16 unique segments from the original time history, the averaged PSD also consists of 15 additional segments created by overlapping neighboring segments by $50 \%$. A schematic is shown in Figure 4.45. Although these segments do not contain unique data, they do provide a slight decrease in the amount of noise. Figure 4.46 is an example of how this averaging technique removes noise in the PSD.

16,384 point time history

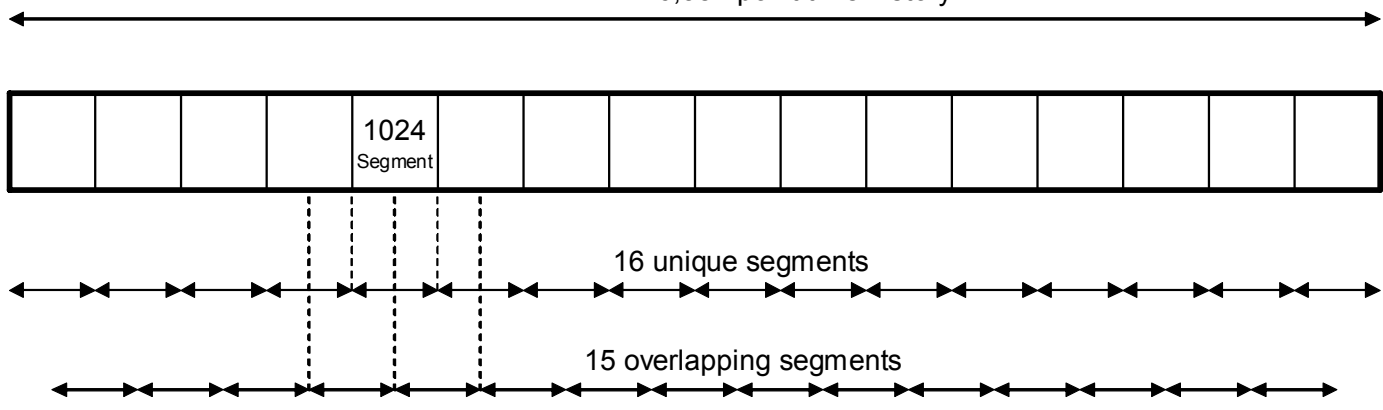

Figure 4.45. Diagram of how data was divided to produce the PSD. 


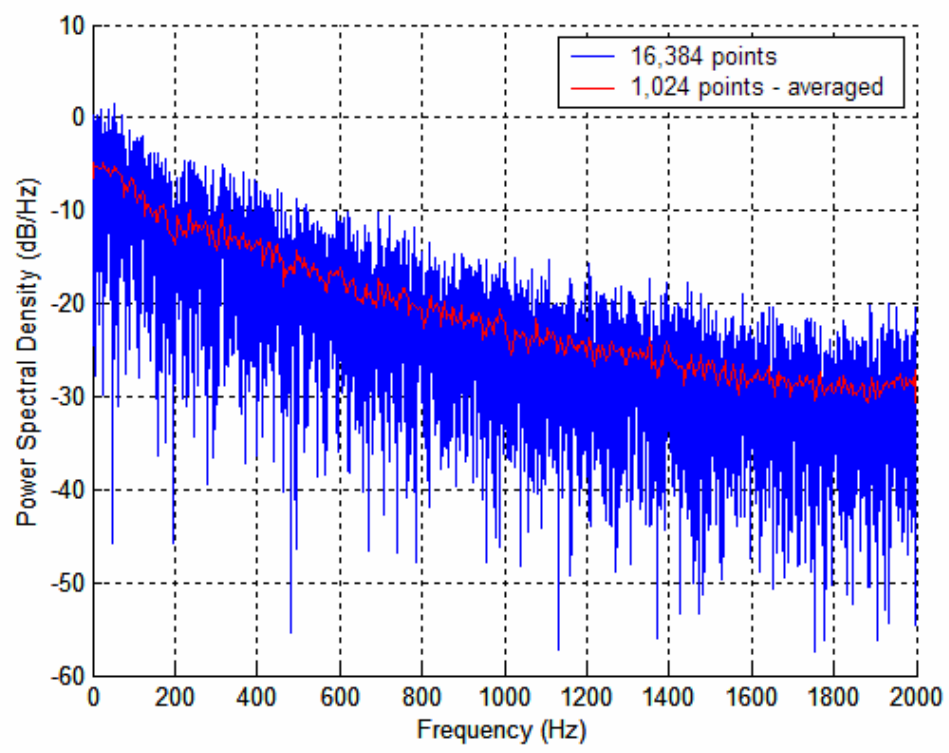

Figure 4.46. Effect of averaging several individual PSDs.

Although the PSD has been significantly improved, the remaining noise still makes it difficult to compare PSDs at different grid points. As a result, the PSD is passed through a low-pass digital filter with a cut-off frequency at $15 \%$ of the sample rate. An example of this smoothing effect is seen in Figure 4.47.

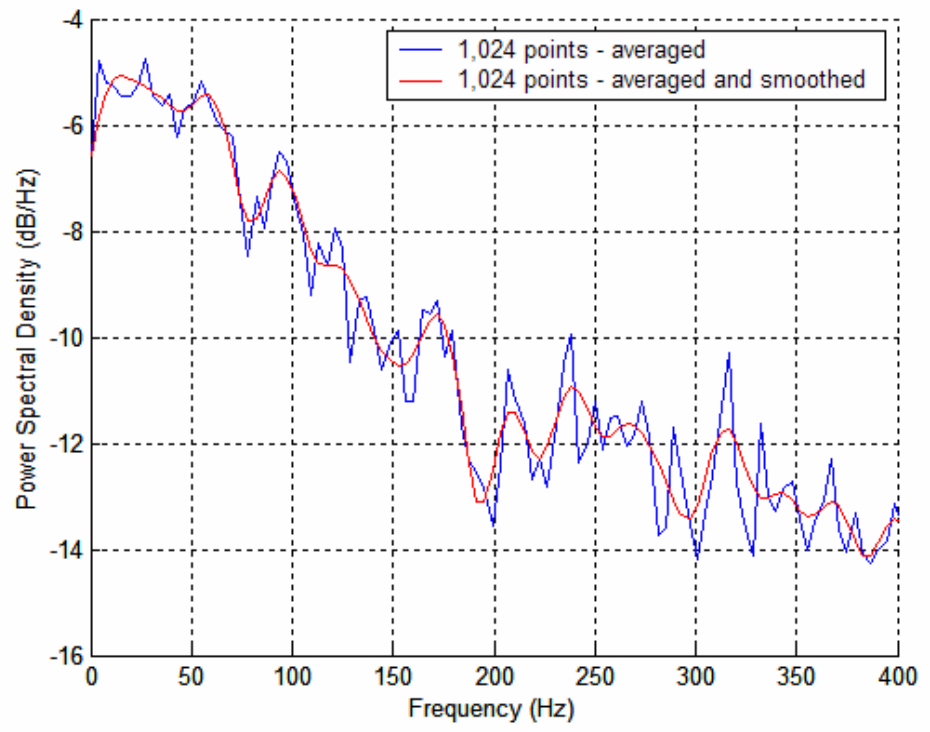

Figure 4.47. Effect of passing the PSD through a low-pass digital filter (smoothed). 


\subsubsection{Power spectral density discussion}

The smoothed power spectral densities of different locations and model configurations are compared. Figure 4.48 shows how the PSDs vary between different configurations at two different locations in the flow field. Unfortunately, there are no distinct frequencies, and the only information obtained qualitatively is that some configurations have PSDs which are offset vertically on the plot. This phenomenon, however, is directly related to the computed standard deviation or normal stress. As a result, further presentation of spectra provides little new information.

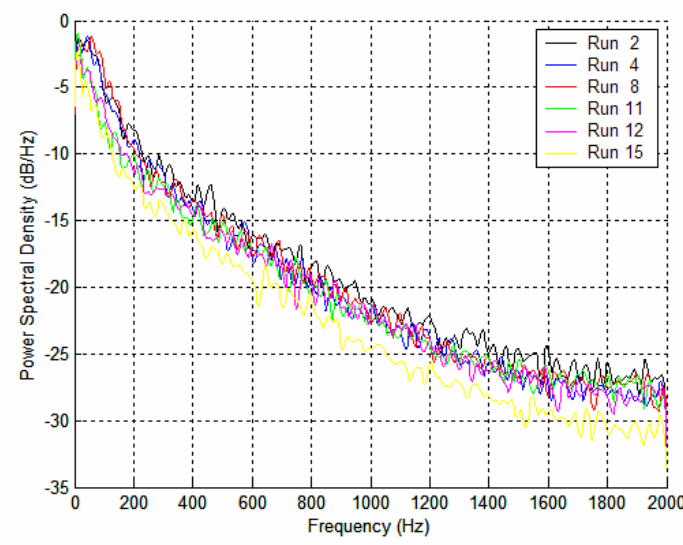

(a)

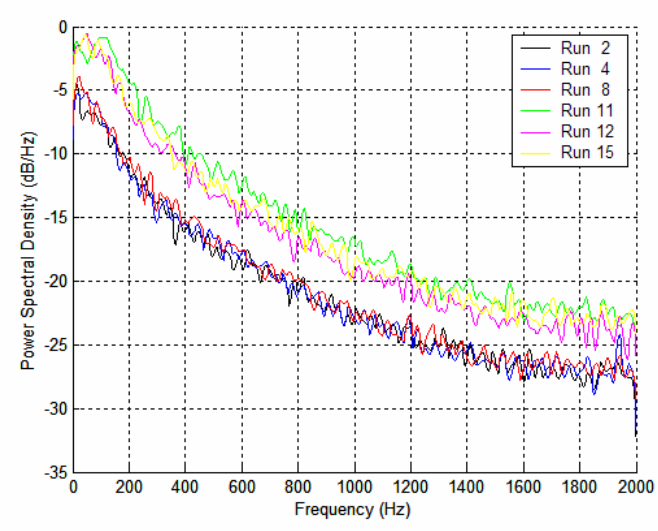

(b)

Figure 4.48. PSDs of selected configurations at: a) $z / h=0.66$ and $2 y / b=0 ; b) z / h=0.66$ and $2 y / b=1.37$.

Because PSDs have not directly yielded any new conclusions, another method of frequency analysis is proposed. First, a further discussion of the physical significance of the PSD is required. As mentioned above, the DFT and the PSD are essentially the same. The main difference between the two is that the PSD plots presented here are scaled by the sampling rate (4000 hertz), yielding units of feet squared per second squared per hertz, or simply decibels per hertz. This is done so that when the PSD curve is integrated across the frequency domain, the value of normal stress is recovered. The standard 
deviation is then simply the square root of this quantity. The PSDs also show that the majority of the energy in the flow originates in the lower frequencies. This low frequency region is important since these fluctuations cause excess pilot workload. As a result, the integration of the PSD up to a certain frequency will allow a numerical comparison of these low frequency fluctuations. Likewise, the unsteady fluctuations in the high frequency domain are also examined.

The frequency defining the difference in low and high frequencies is called the cut-off frequency. Determination of this parameter is difficult and several methods are proposed. First, the frequency which divides the PSD into approximately equal areas can be chosen. Another method involves using the characteristic shedding frequency of the model (even though this shedding is not observed). A third method involves the physics of turbulence. All three of these methods are considered.

The first method yields an even breakdown of energy. Because there are no distinct frequencies, the PSD could be split into two equal areas with the dividing line being the cut-off frequency. This avoids the defined low frequency unsteadiness becoming similar in magnitude to the total unsteadiness. The second method requires consideration of bluff body aerodynamics. Given the Reynolds number of the experiment, the Strouhal number is approximated. However, because the Reynolds number is near the supercritical regime, periodic shedding is not achieved. ${ }^{39}$ This is a possible explanation for the lack of detection of large coherent structures, and hence a dominant frequency. The third method considers the physics of turbulence. Figure 4.49 shows a typical frequency spectrum on a log-log scale. Turbulence consists of three distinct regions in high Reynolds number flows. ${ }^{40}$ The first is the energy containing 
region which consists of the lowest frequencies. Second, the inertial subrange exists at moderate frequencies. This region is characterized by Kolmogorov's hypothesis and spectra which fall off at a rate proportional to frequency raised to the $-5 / 3$ power. Larger eddies are broken up into smaller eddies by inertial effects. Third, the dissipation region is where the smallest eddies are broken up due to viscous effects. This region cannot be seen in the data due to the limited sampling frequency. Considering these three spectrum ranges, a logical cut-off would be at the transition to the inertial subrange because it is desired to isolate the low, energy-containing frequencies.

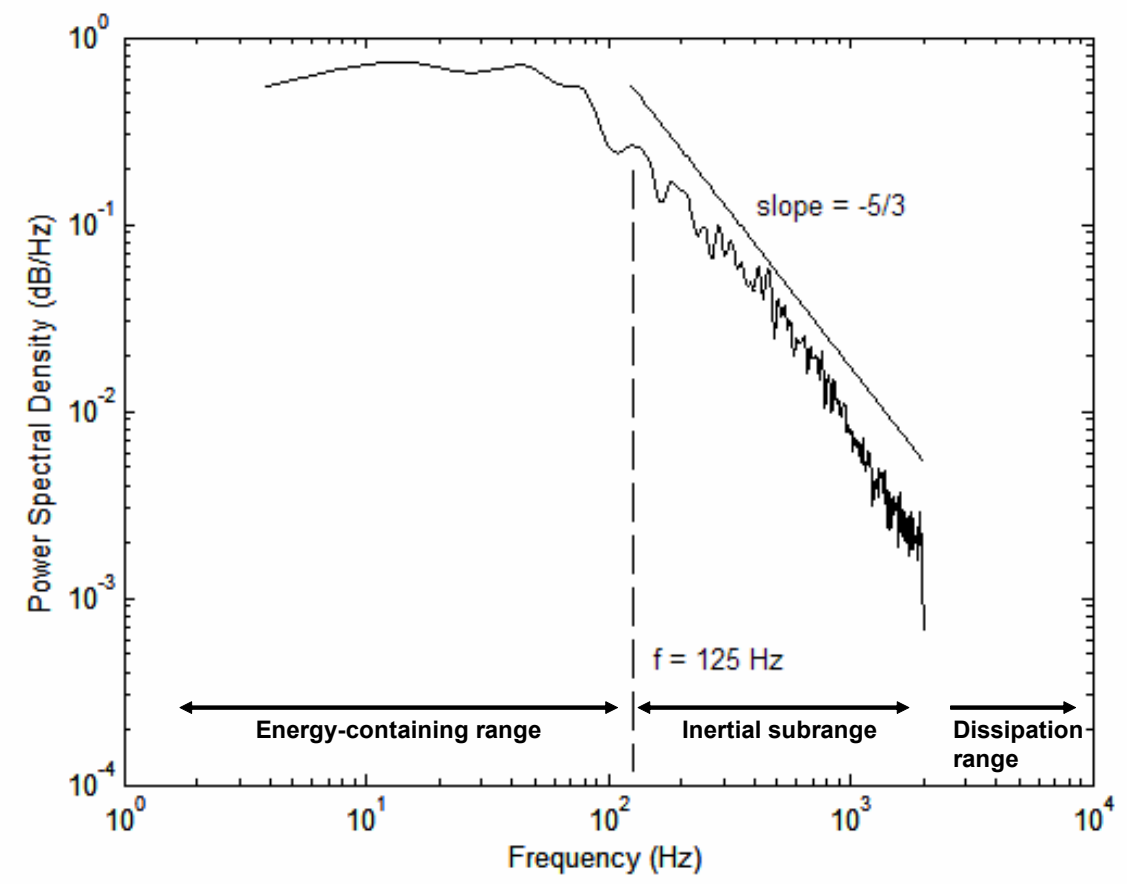

Figure 4.49. Velocity spectra following Kolmogorov's hypothesis.

After considering these three methods, the cut-off frequency was chosen by a combination of the first and third methods. Recall that the original time histories were divided into segments of 1,024 samples, producing PSDs with a sensitivity of about 4 hertz. As a result, the cut-off frequency should be on the order of 100 hertz to integrate 
over a reasonable number of points. Compromising between obtaining equal areas beneath the PSD and using a sufficiently large number of points for the integration, a cutoff frequency of 125 hertz was chosen. This is also near the beginning of the inertial subrange as mentioned above.

The PSD from each grid point is numerically integrated to the cut-off frequency ( 0 to 125 hertz); the trapezoidal method is used on the unfiltered PSD. The ratio of the standard deviation at each grid point due to low frequencies to the total standard deviation is shown in Figure 4.50. In the wake regions, the lower frequencies dominate the turbulence, whereas the freestream is dominated by higher frequency turbulence.

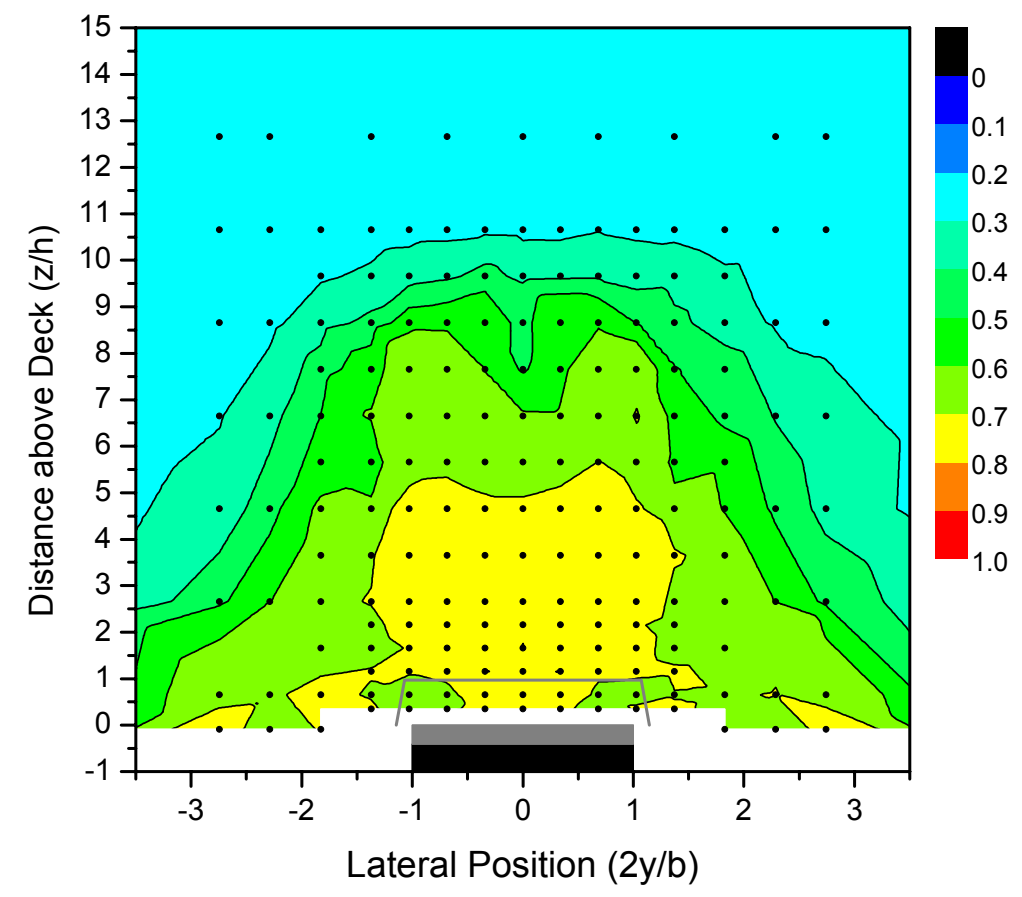

Figure 4.50. Ratio of the standard deviation due to low frequencies to the total standard deviation for the baseline configuration.

\subsubsection{Frequency-separated standard deviation contours}

Contour plots similar to those in Section 4.2.1 are generated from the low and high frequency standard deviation values. Figure 4.51 shows the baseline velocity 
standard deviation in both the low and high frequency domains. Note that if the values at each point on these two plots were summed, the standard deviation contours presented in Section 4.2.1 would be recovered. The contour levels and colors are exactly the same as those presented earlier, going from $0 \%$ (blue) to $22 \%$ (red) of the freestream. These two plots serve as a new baseline for comparison between devices. The lower frequencies are dominant, but increased high frequency fluctuations are present near the sides of the ship.

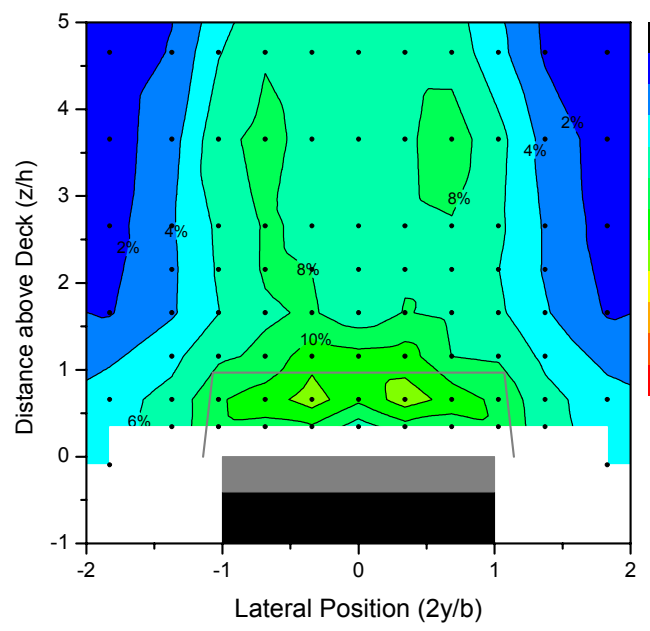

(a)
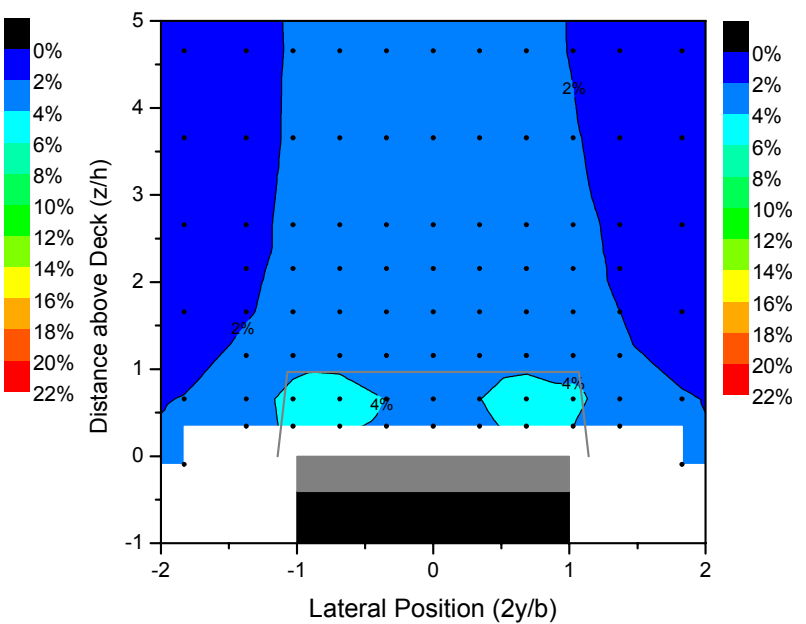

(b)

Figure 4.51. Baseline velocity standard deviation for: (a) the $0-125 \mathrm{~Hz}$ domain; (b) the $125-2000$ Hz domain.

This frequency domain breakdown is consistent with the conclusions for the porous surface configurations. Figure 4.52 shows the contours for the case with a porous flight deck and injection from the hangar face (run 8). Other porous surface configurations experience similar changes when separated into low and high frequencies. Note again that an increase in high frequency unsteadiness occurs near the sides on the hangar. The region of high frequency unsteadiness does not occur at the top of the hangar in the middle because the oncoming flow has less energy. This is a result of the lower mean velocity near the centerline of the ship due to the wake of upstream bodies 
(the ship's mast, bridge, stacks, etc.). Examining standard deviation contours of the fence configurations verifies this statement. Figure 4.53 shows the serrated porous fence configuration (run 11). The unsteady regions in this case are pushed upward and outward, however, the high frequency unsteadiness is greater in areas with a higher mean velocity. Other fence configurations exhibit similar characteristics.

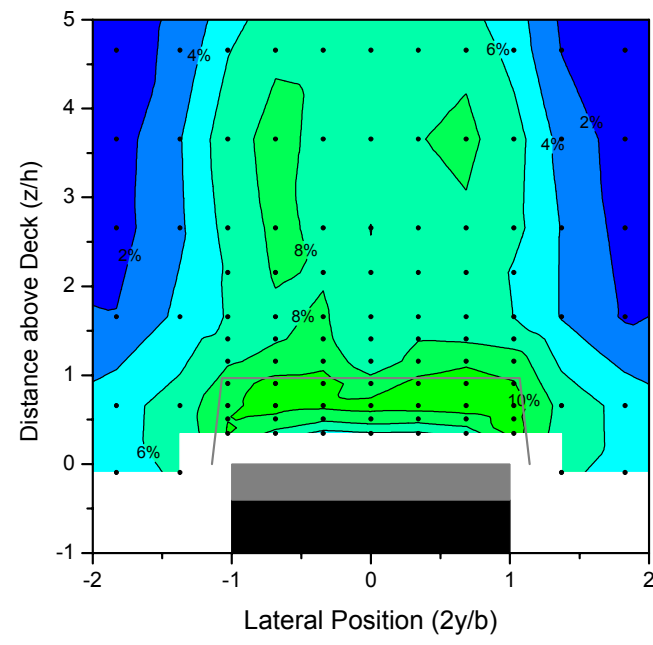

(a)
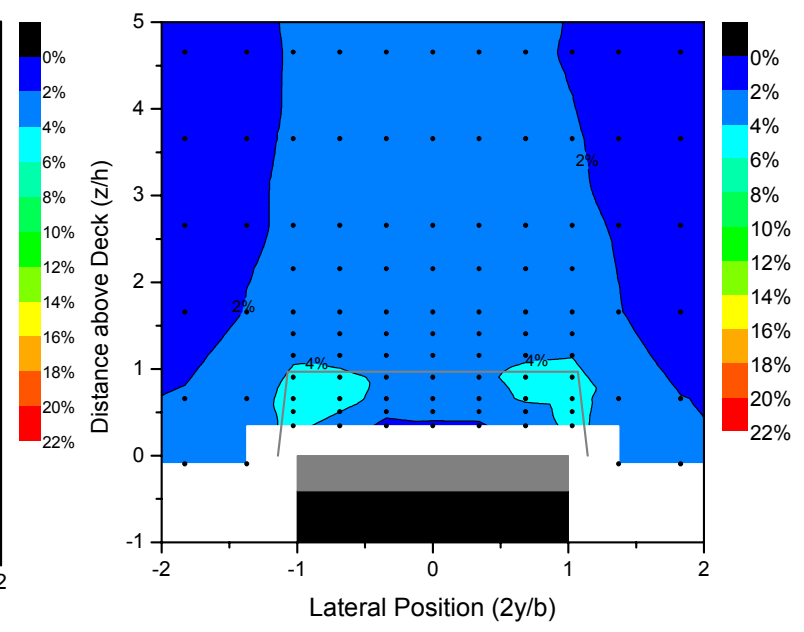

(b)

Figure 4.52. Velocity standard deviation for the porous flight deck with blowing from the hangar face for: (a) the $0-125 \mathrm{~Hz}$ domain; (b) the $125-2000 \mathrm{~Hz}$ domain.

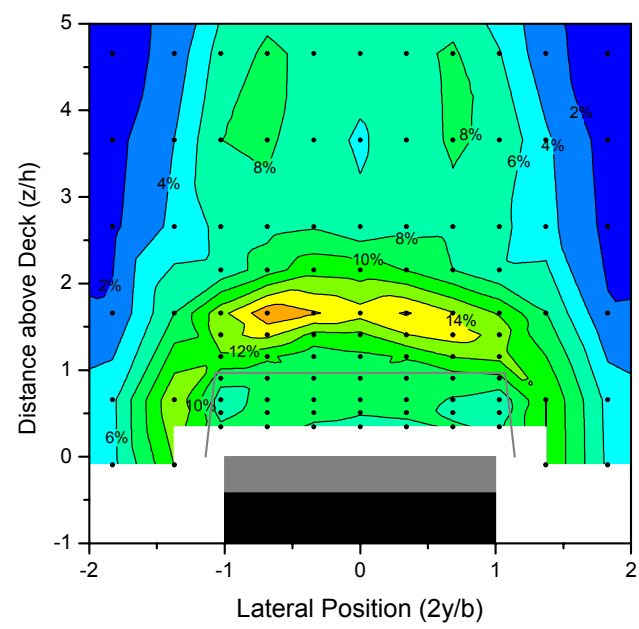

(a)

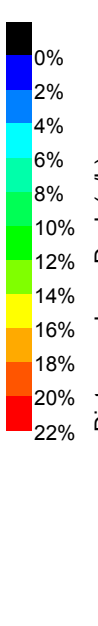

Figure 4.53. Velocity standard deviation for the porous serrated fence configuration for: (a) the 0 $125 \mathrm{~Hz}$ domain; (b) the $125-2000 \mathrm{~Hz}$ domain.

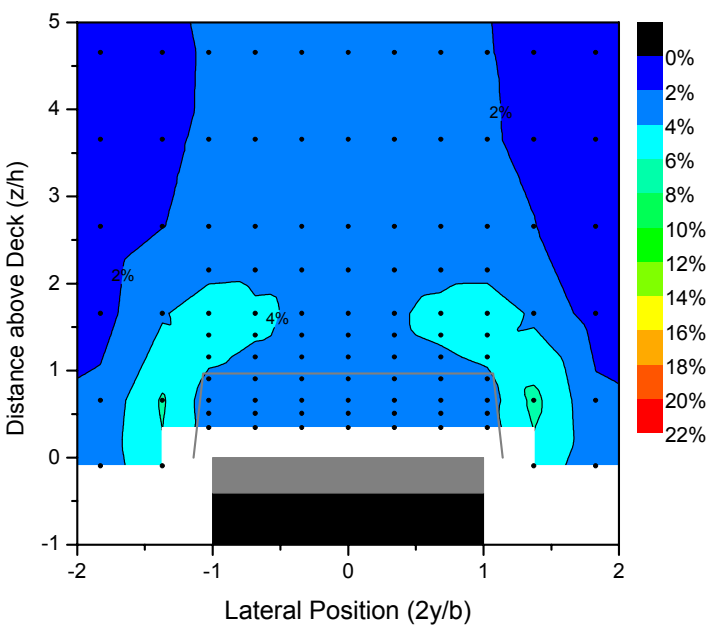

(b) 


\subsubsection{Alternative frequency breakdown}

Another method to determine the cut-off frequency is to consider the frequency scaling between full scale and the wind tunnel tests. A model scale of 1:144 multiplies by a velocity scale of approximately 1:1.5 yielding a frequency scaling of 216:1. Also note that frequencies between 0.1 and 2 hertz are responsible for increased pilot workload. ${ }^{33}$ Therefore, this method implies the frequency domain of interest in this investigation is 21.6 to 432 hertz. The unsteadiness now responsible for pilot workload can be obtained by integrating the PSD over these frequencies. Unfortunately because the McRuer ${ }^{33}$ paper was discovered after analysis was completed, this new frequency range was unable to be completed for inclusion in this document. However, because this method includes much of the energy containing region, it is expected that it will produce contours similar to the low frequency contours shown above.

\subsubsection{Frequency analysis summary}

Conclusions of the frequency analysis are summarized. First, the power spectral densities of velocity time histories do not reveal any dominate frequencies. The only difference between configurations is that more unsteadiness causes the y-intercept of the PSD to increase. Second, the spectra are used to separate the low frequency unsteadiness from the high frequency unsteadiness by selecting a cut-off frequency. Upon replotting the contours in Section 4.2.1, conclusions regarding the performance of control devices were not changed. Finally, these contours show that high frequency unsteadiness is more common in regions of higher mean velocity. This implies that the fence configurations successfully break up some of the larger flow structures and shift them into the higher 
frequency domain, but only in regions of higher mean velocity near the sides of the ship. As a result, a future investigation should include configurations with flow control devices (fences for example) on the sides of the hangar only. 


\section{RESULTS AND DISCUSSION: 20 DEGREES WIND-OVER-DECK}

Because ships rarely cruise directly into a headwind, nonzero wind-over-deck (WOD) angles must be considered. The focus of the investigation was to evaluate several flow control devices to determine which was most effective in decreasing pilot workload. It was desired to test a wide range of devices rather than focus on the effects of varying the WOD angle. As a result, only two flow control devices were chosen for evaluation at 20 degrees WOD. The best performing devices from each category in Chapter 4 were the configuration with porous ship surfaces and injection from the hangar face (run 8) and the reticulated foam fence configuration (run 15). Note that the model was rotated about the stern in the wind tunnel, but the measurement system and traverse remained orthogonal to the tunnel. Consequently, the data plane was skewed with respect to the flight deck; however, the axial position of the measurement plane still passed through the middle of the flight deck. Because of this skewness, the contour plots at this WOD angle include a ship cross-section that is slightly wider than the 0 degrees WOD case (the same non-dimensionalization parameters are used). Recall from the uncertainty analysis that the measurements in regions of high turbulence intensity should be analyzed qualitatively only. Because the ship is yawed 20 degrees, this region is much larger than for the 0 degrees WOD case. 


\subsection{Mean velocity}

\subsubsection{Mean velocity contours}

Consider the baseline mean velocity contour plot for the 20 degrees WOD case shown in Figure 5.1. Because the hangar face is upstream of the data plane, its projection is offset to the right as shown in the figure. The contour levels and colors are the same as in Section 4.1 .1 ranging from $0 \%$ (blue) to $104 \%$ (red) of the freestream velocity. The large velocity deficit on the right side of the figure is caused by the mast, antennas, stacks, etc. Interestingly, the lowest mean velocity is measured on the windward side of the side of the flight deck.

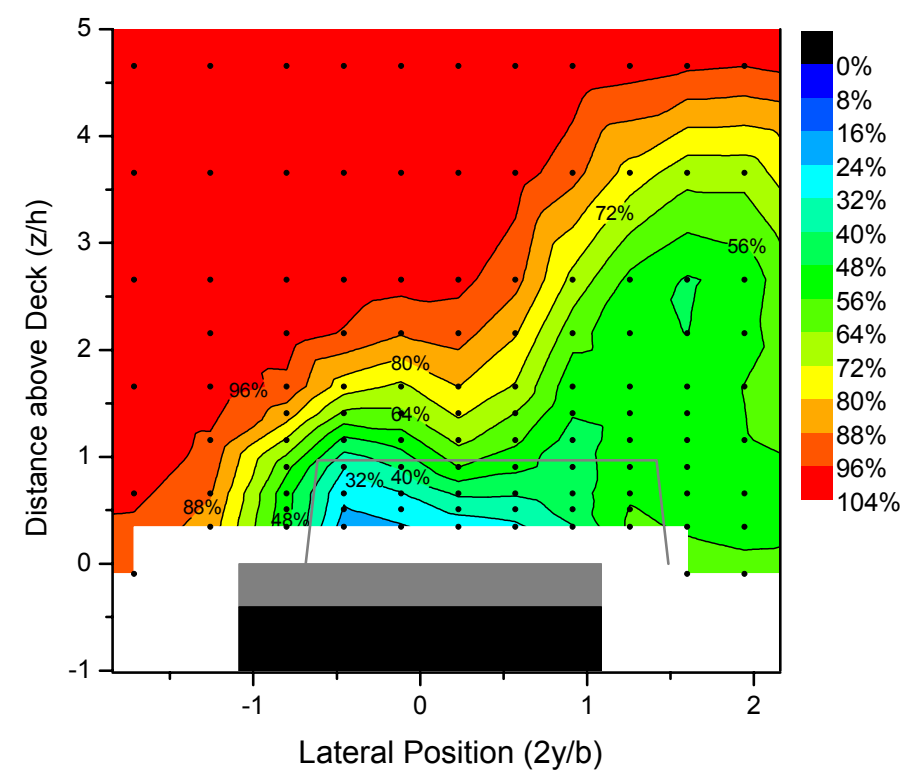

Figure 5.1. Mean velocity for the 20 degrees WOD baseline configuration. The contours are in percent of freestream velocity.

The mean velocity contours of the two flow control devices tested at this WOD angle are shown in Figures 5.2 and 5.3. The reticulated foam fence (run 17) has caused a much larger region of low speed flow behind the hangar. Unfortunately, the velocity gradients have also become much larger, especially on the windward side of the ship. 
The fence on the leeward side appears to have little effect on the mean as the flow on the starboard side of the ship already has a massive velocity deficit. The other configuration (run 18) shows a flow field more similar to the baseline, however the velocity behind the hangar has decreased.

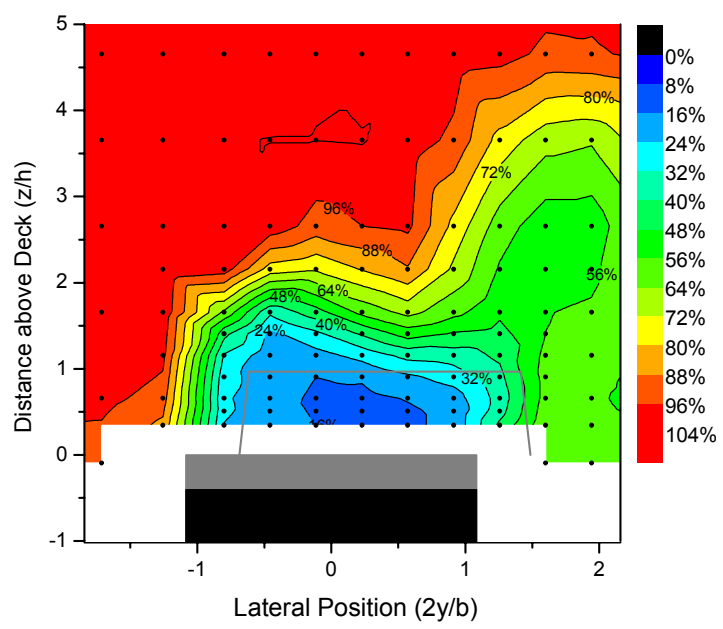

Figure 5.2. Mean velocity of reticulated foam fence configuration (run 17).

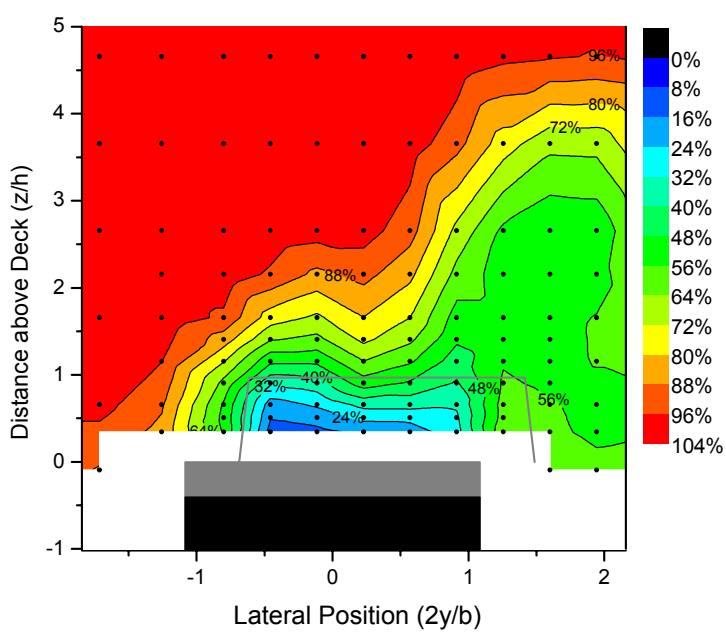

Figure 5.3. Mean velocity of porous surfaces with injection configuration (run 18).

\subsubsection{Mean velocity line plots}

The features observed in the contour plots are better quantified by examining line plots of data in the measurement plane. Figures 5.4 and 5.5 show a horizontal and vertical slice of data. The location of the horizontal slice is the same height of those in the prior chapter. This height $(z / h=0.66)$ corresponds to rotor height at a hover a few feet above the deck. The vertical cut was taken at $2 y / b=0.23$. This slightly off-center coordinate was chosen because of the nonzero WOD angle. The data shows that the foam fence causes the mean velocity to decrease by a factor of three at many spanwise locations across the flight deck. The downside is the very steep gradient on the windward side of the ship. Although this feature is not located over the center of the flight deck at 20 degrees WOD, the steep velocity gradient is likely to move towards the leeward side 
as the WOD angle is increased. Figure 5.5 shows the reduction in mean velocity for the foam fence in the vertical direction. Above $1.5 \mathrm{~h}$, the velocity gradient is severe. It is difficult to determine how larger WOD angles may affect the behavior in the vertical direction.

In summary, the two devices evaluated have similar trends although the foam fence has a more pronounced effect. As discussed in Chapter 4, the benefits of reducing the mean flow behind the hangar must be weighed with the potential hazard of worsening the velocity gradient around the landing region. The gradient is the most severe on the windward side of the ship.

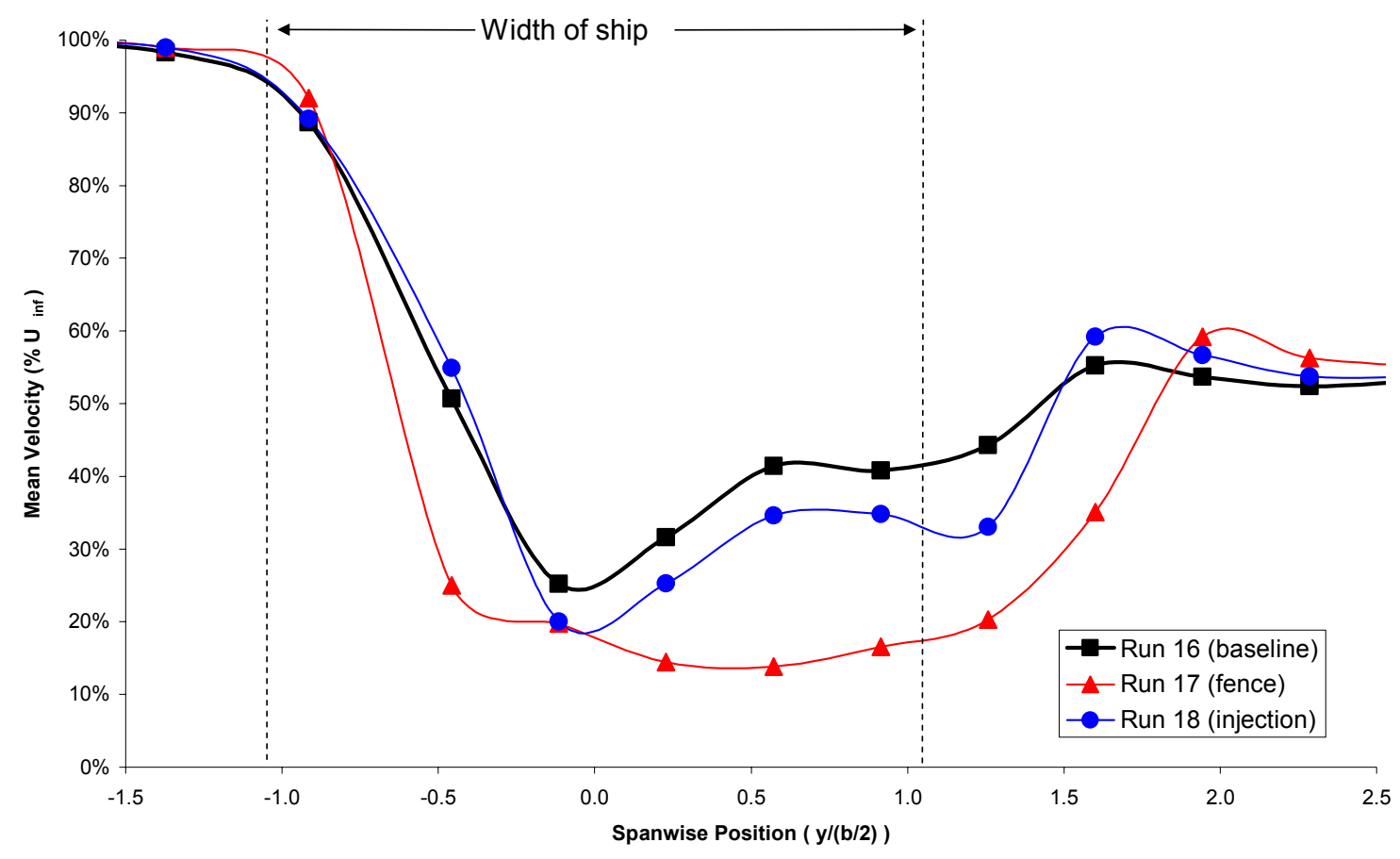

Figure 5.4. Mean velocity at low hover $(z / h=0.66)$ for 20 degrees WOD case. 


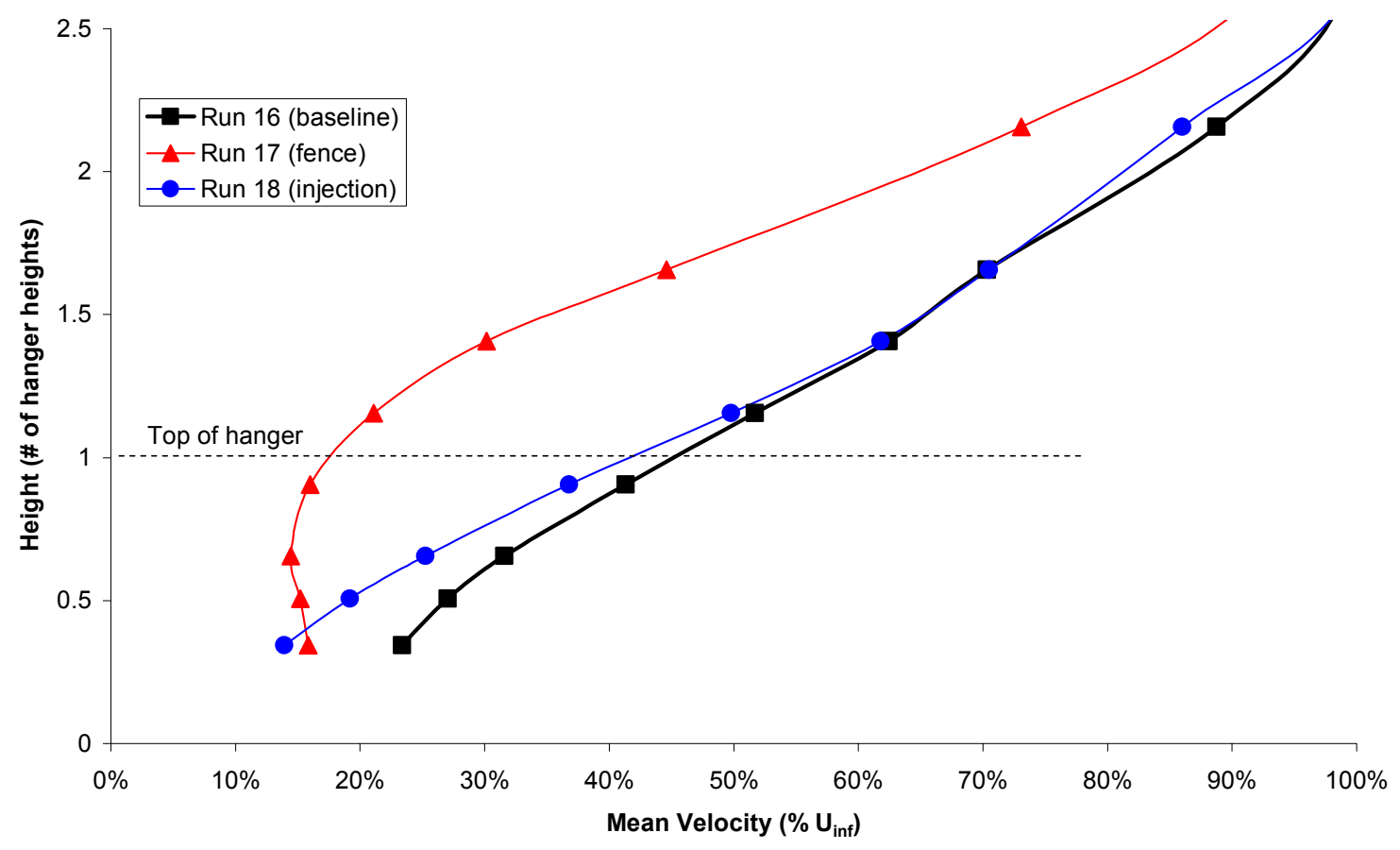

Figure 5.5. Mean velocity near the vertical centerline $(2 y / b=0.23)$ for 20 degrees WOD case.

\subsection{Velocity standard deviation}

\subsubsection{Velocity standard deviation contours}

The standard deviation of the velocity time history at each grid point is again shown in the form of contour plots. Figure 5.6 is the baseline for the 20 degrees WOD case. The contours are the same as in Section 4.2.1, ranging from $0 \%$ (blue) to $22 \%$ (red) of the freestream velocity. The figure shows that the flow on the windward side is generally more unsteady. This maximum unsteadiness occurs downstream of the side of the hangar and extends across the top half of the hangar. When the reticulated foam fence was added (Figure 5.7), the flow field is altered substantially. The unsteadiness from the windward side edge of the hangar is reduced by about $30 \%$. Thus, it is concluded that a steep velocity gradient does not necessarily imply a region of increased 
unsteadiness. (Recall the steep mean velocity gradient on the windward edge of the hangar in Figure 5.2.). Additionally, the peak unsteadiness along the top of the hangar has been pushed upward without amplification, and the maximum unsteadiness did not increase as it was moved away from the flight deck. Finally, the foam fence has reduced the unsteadiness behind the hangar significantly, just as it did during the 0 degree WOD test. It is concluded that this device has improved the flow field.

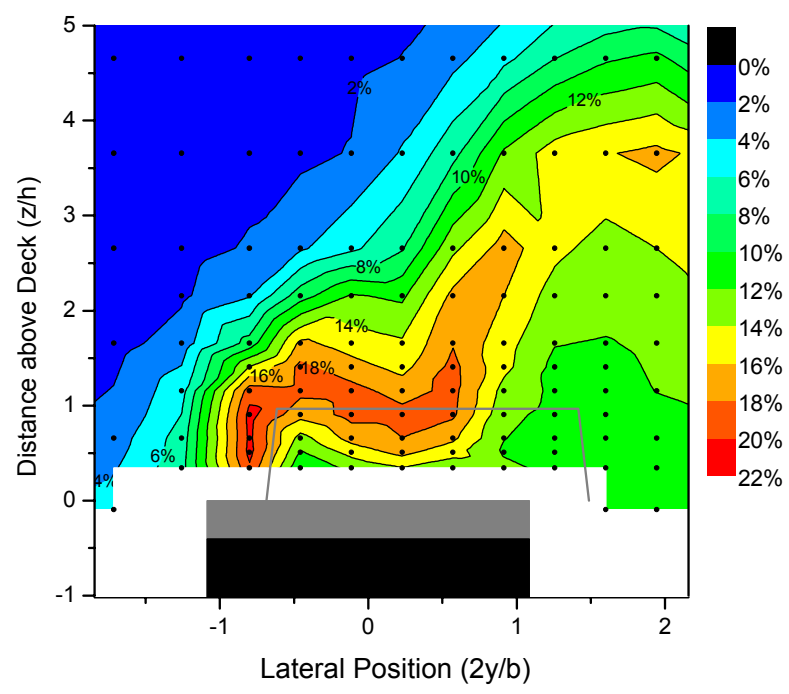

Figure 5.6. Velocity standard deviation for the 20 degrees WOD baseline configuration. The contours are in percent of freestream velocity.

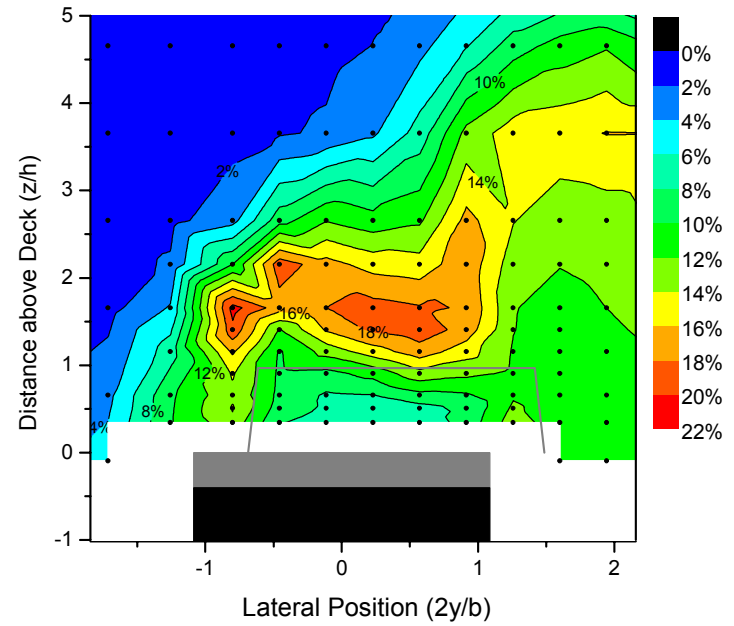

Figure 5.7. Velocity standard deviation of reticulated foam fence configuration (run 17).

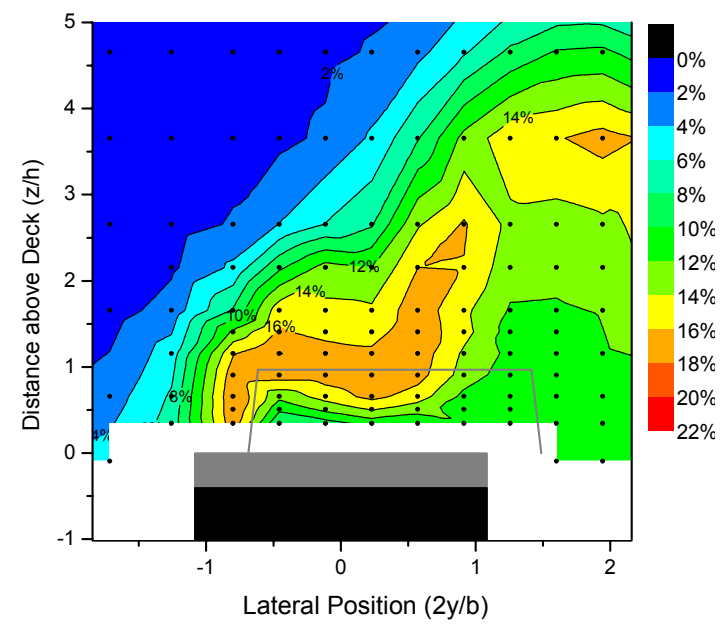

Figure 5.8: Velocity standard deviation of porous surfaces with injection configuration (run 18). 
The configuration tested in run 18 (porous surfaces with injection) also showed flow field improvements. Figure 5.8 shows that although the flow control technique did not result in shape changes of the contours, the unsteadiness was reduced along the top of the hangar and windward side.

\subsubsection{Velocity standard deviation line plots}

The contour plots were analyzed in a set of line plots. Figure 5.9 shows the distinct advantages of the reticulated foam fence for a horizontal slice of data in the measurement plane. Although run 18 has reduced the unsteadiness slightly, it is clear that the foam fence is superior. Reductions in unsteadiness across the flight deck exceed $50 \%$ at some spanwise locations. Figure 5.10 shows similar reductions along a vertical slice through the data plane. It is important to note that the foam fence "pushes" the unsteadiness upward without an increase. Recall that for the 0 degree WOD case all the fences caused an increase in unsteadiness as the shear layer was pushed upward (Figure 4.27). As a result, it is concluded that the foam fence is more advantageous at nonzero WOD angles. 


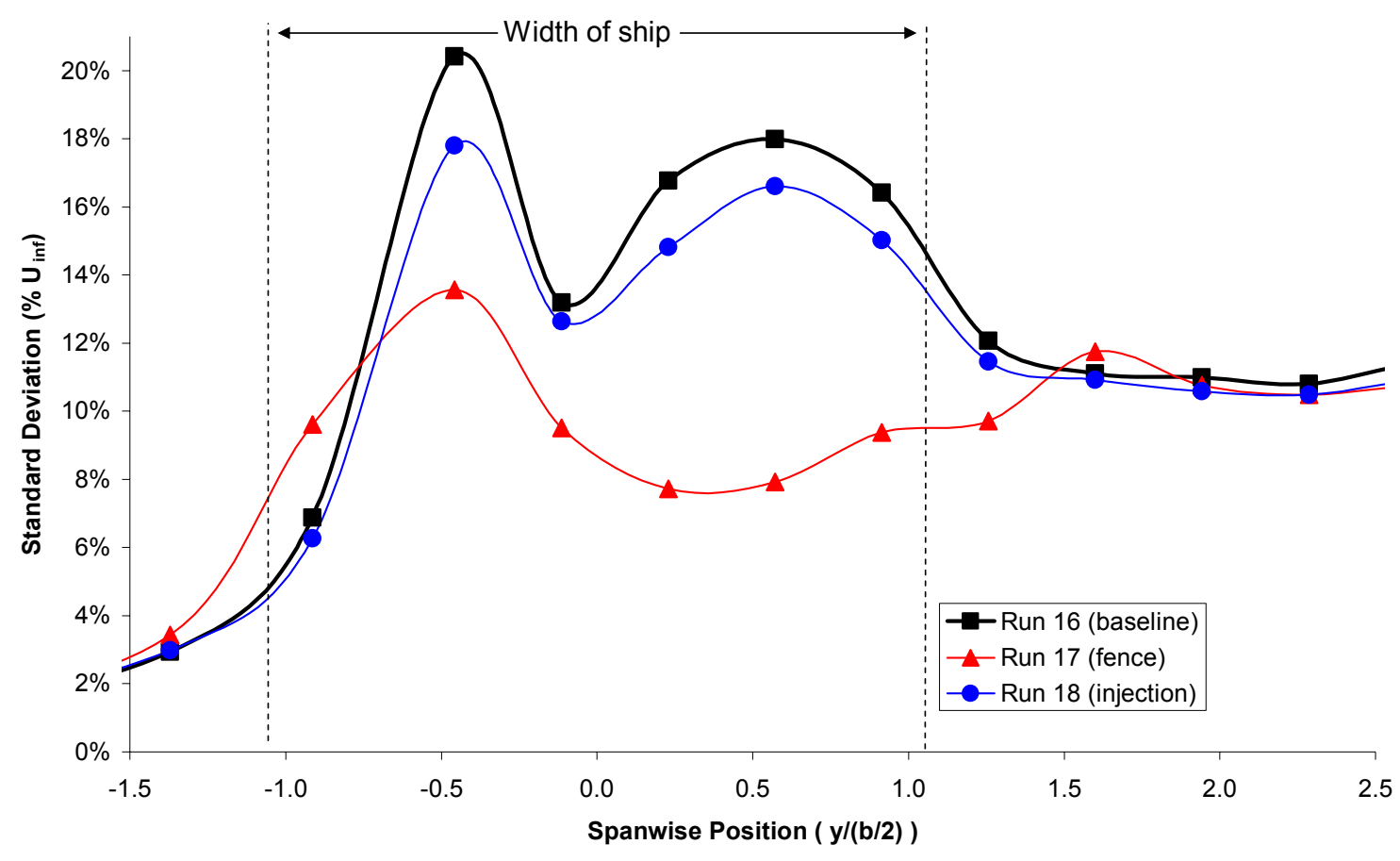

Figure 5.9. Velocity standard deviation at low hover $(z / h=0.66)$ for 20 degrees WOD case.

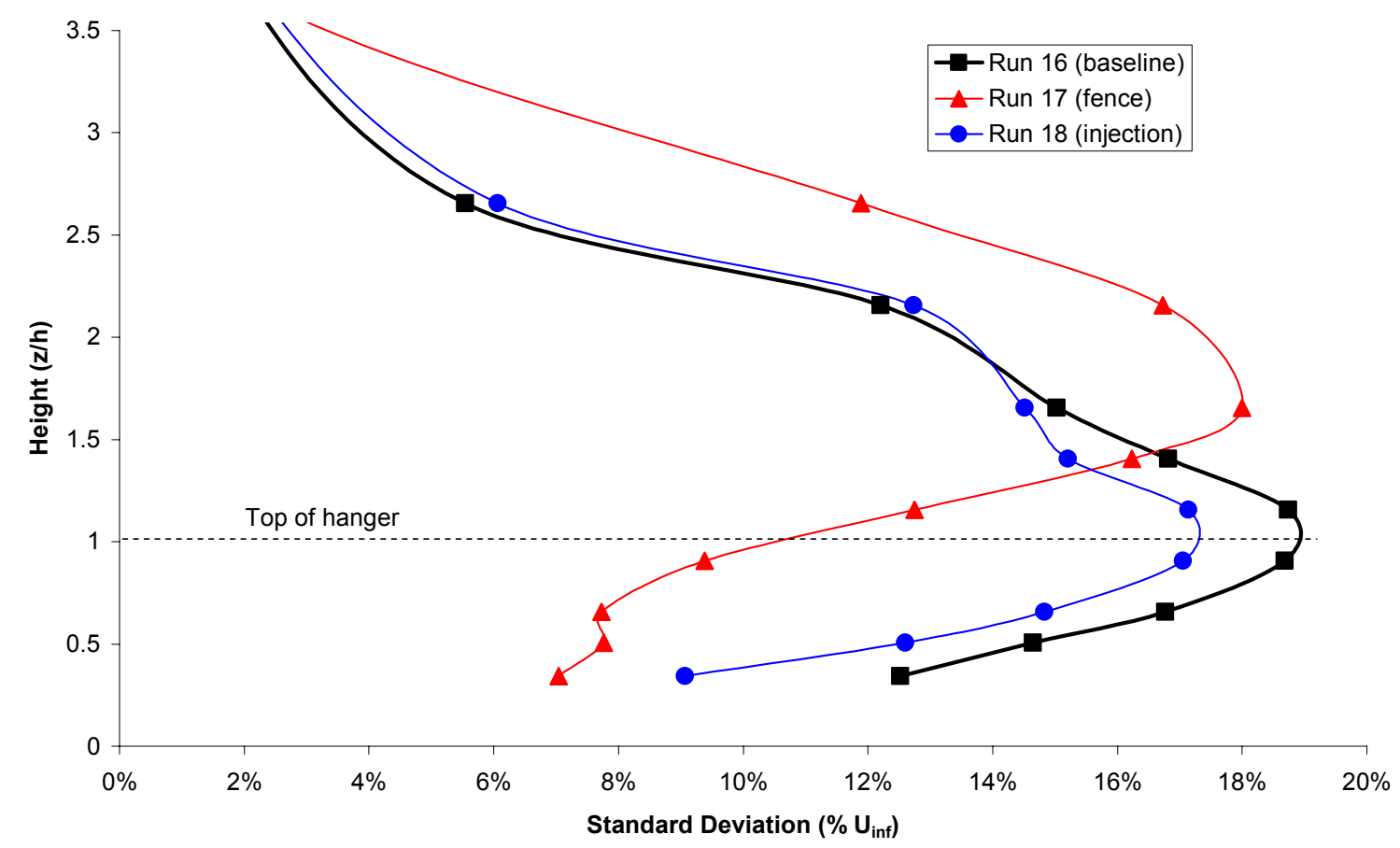

Figure 5.10. Velocity standard deviation near the vertical centerline $(2 y / b=0.23)$ for 20 degrees WOD case. 


\subsection{Turbulence intensity}

Recall from Section 4.3 that standard deviation is a relative measure of unsteadiness. Also recall that desirable characteristics for helicopter/ship operations include low mean velocity with a minimal amount of unsteadiness. Because turbulence intensity divides these two parameters, it may not be the best evaluation criteria. Regardless, analysis is included for completeness.

\subsubsection{Turbulence intensity contours}

Figure 5.11 shows the baseline configuration for the 20 degrees WOD case. The contours range from a $0 \%$ (blue) to $65 \%$ (red) turbulence intensity. For both of the devices tested at this WOD angle (Figures 5.12 and 5.13), the contour plots lead one to believe that the devices affect the flow field adversely. The maximum turbulence intensity is increased for both control techniques, and this peak occurs close to the flight deck.

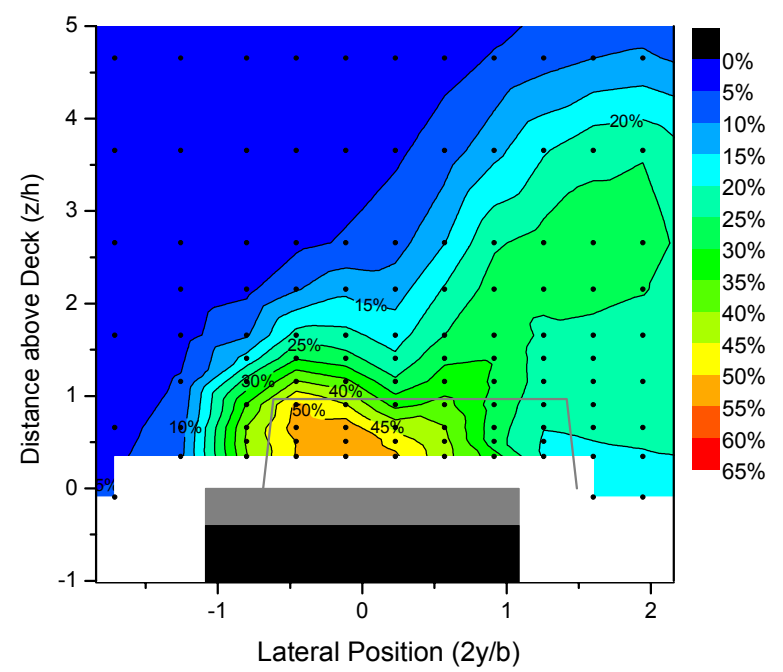

Figure 5.11. Turbulence intensity for the 20 degrees WOD baseline configuration. 


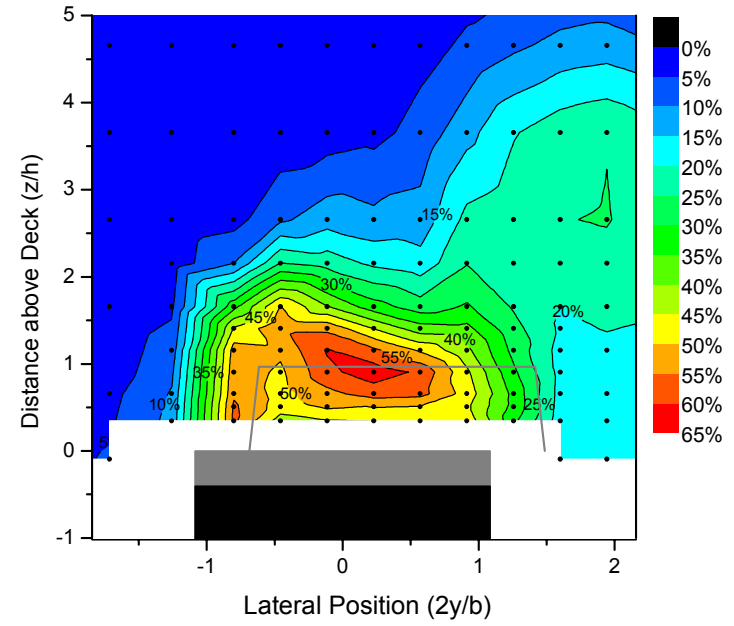

Figure 5.12. Turbulence intensity of the reticulated foam fence configuration (run 17).

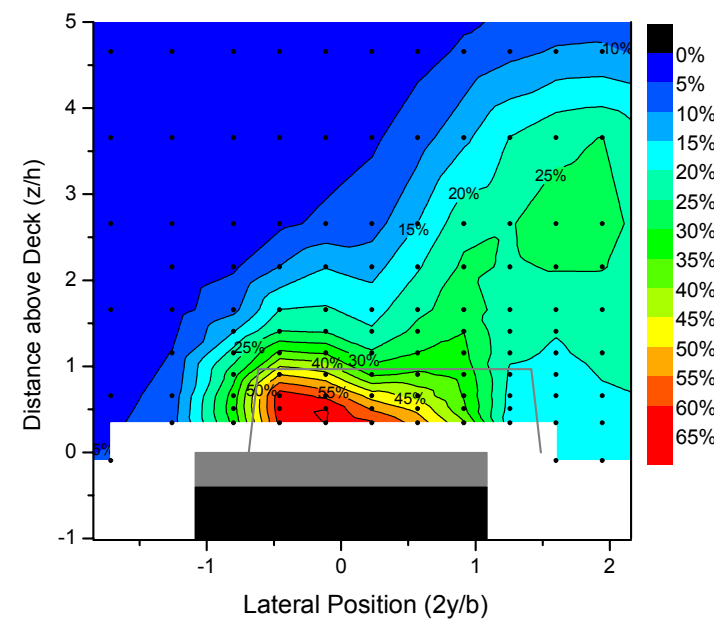

Figure 5.13. Turbulence intensity of the porous surfaces with injection configuration (run 18).

\subsubsection{Turbulence intensity line plots}

Line plots taken through the data plane also show how turbulence intensity misrepresents the performance of control devices. Figures 5.14 and 5.15 show the intensity at a horizontal and vertical slice respectively. In both cases, the turbulence intensity has a net increase over the baseline configuration. 


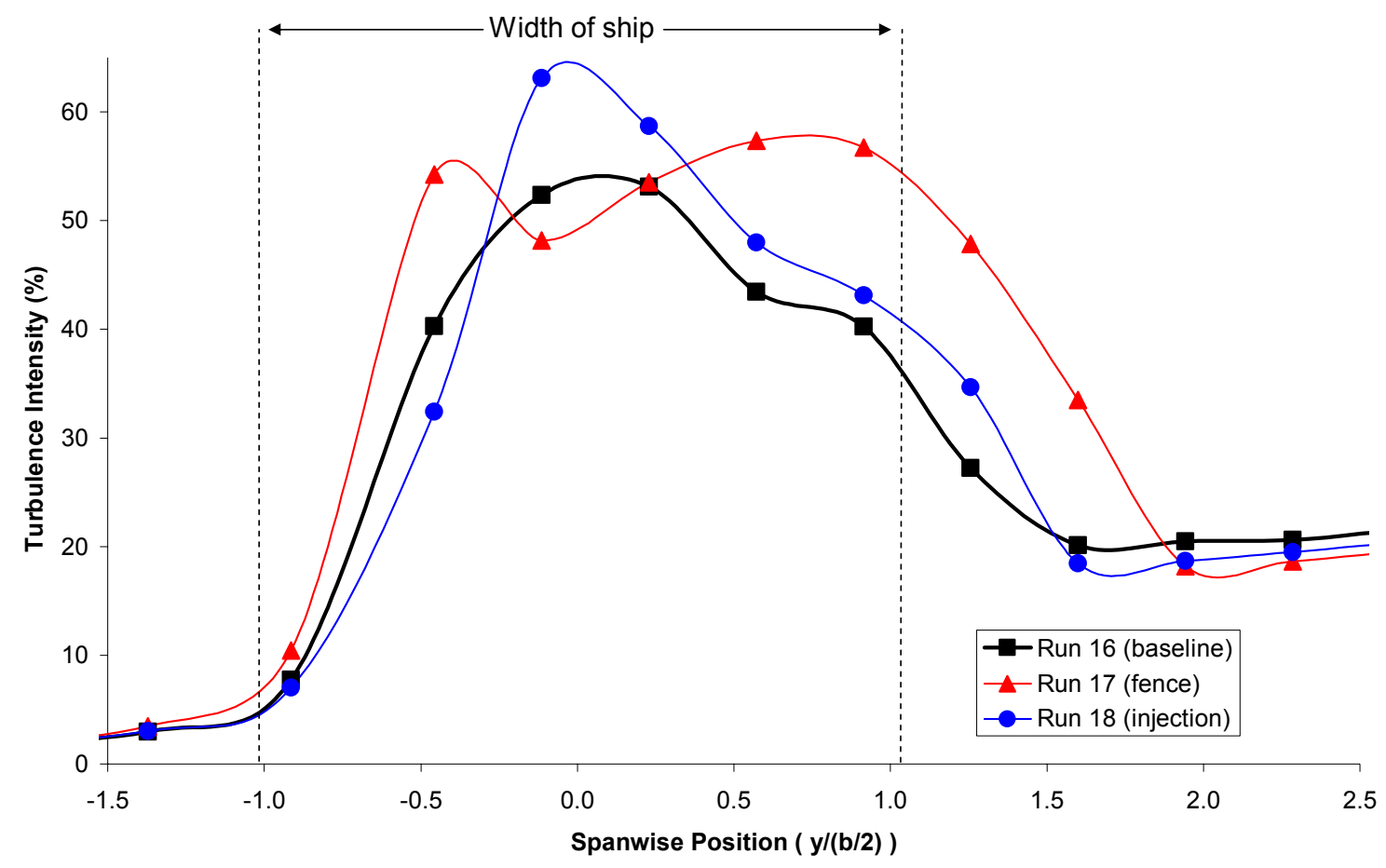

Figure 5.14. Turbulence intensity at low hover $(\mathrm{z} / \mathrm{h}=\mathbf{0 . 6 6})$ for 20 degrees WOD case.

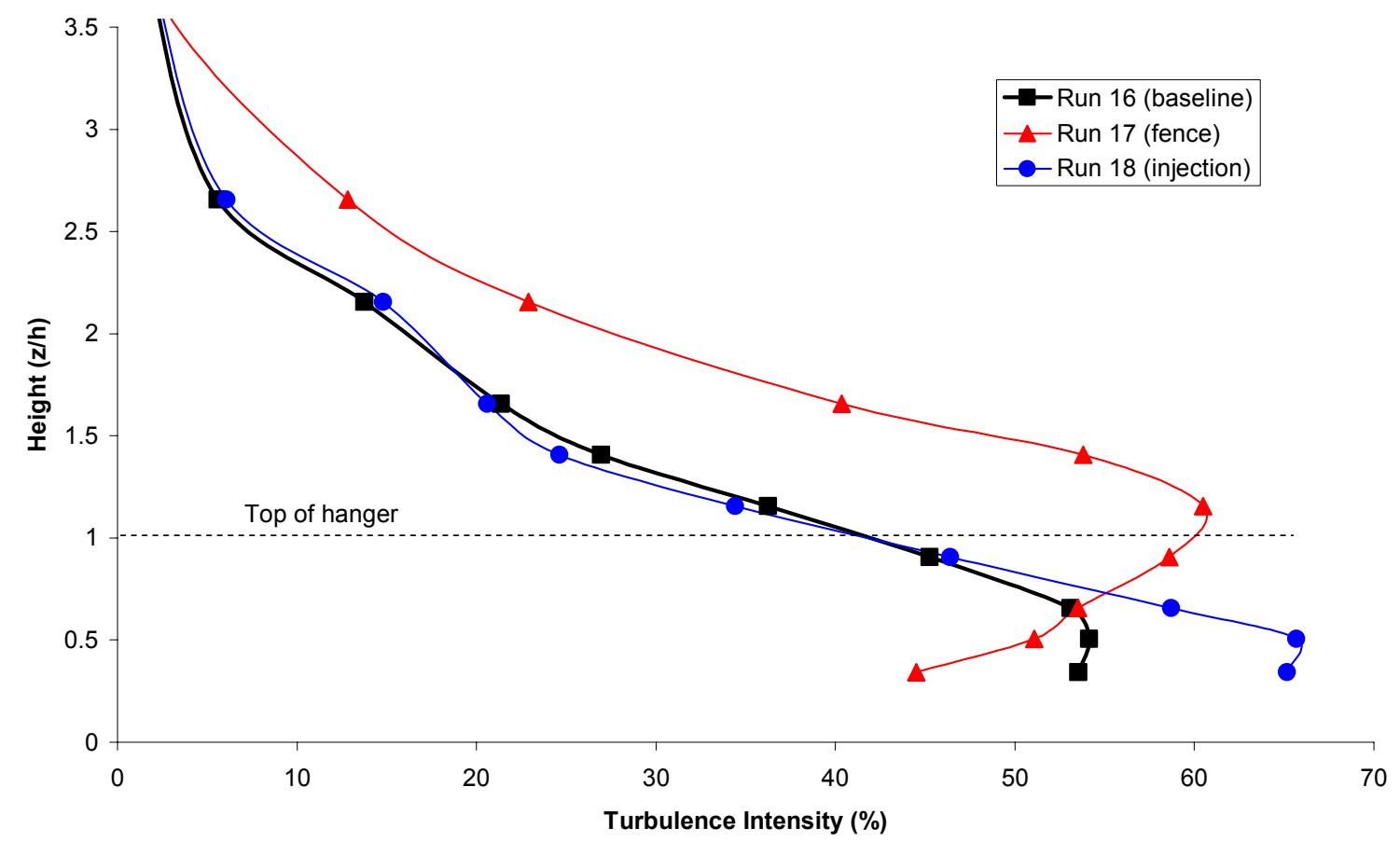

Figure 5.15. Turbulence intensity near the vertical centerline $(2 \mathrm{y} / \mathrm{b}=0.23)$ for 20 degrees WOD case. 


\subsection{Average unsteadiness in landing area}

The average unsteadiness parameter is calculated for the configurations at 20 degrees WOD. This evaluation metric was derived in Section 4.4. Results show that the reticulated foam fence (run 17) caused a $12.11 \%$ reduction in unsteadiness in the landing region. This is significant as it is the largest reduction calculated with this parameter. Run 18 (porous surfaces with injection from the hangar face) had an $8.28 \%$ decrease in unsteadiness. Recall that the maximum reduction of unsteadiness in the landing area at 0 degrees WOD was only $6.56 \%$. As a result, future ship flow control device studies may show better results by comparing devices at nonzero WOD angles. In hindsight this seems logical since a 0 degree WOD occurs far less than other angles.

\subsection{Frequency analysis}

A frequency analysis of the 20 degree WOD configurations was conducted using the same methods in Section 4.5. Power spectral density (PSD) plots take the same shape of the 0 degree WOD cases. The standard deviation contour plot was then separated into a low frequency domain and a high frequency domain using the defined cut-off frequency of 125 hertz. The unsteadiness of the baseline configuration for the 20 degrees WOD case is shown in Figure 5.16. Unlike the 0 degrees WOD case, the maximum high frequency unsteadiness does not occur downstream of the sides of the hangar. Instead, there is simply a large region of moderate high frequency unsteadiness. The low frequency unsteadiness contours take the shape of the total standard deviation shown in Figure 5.6. 


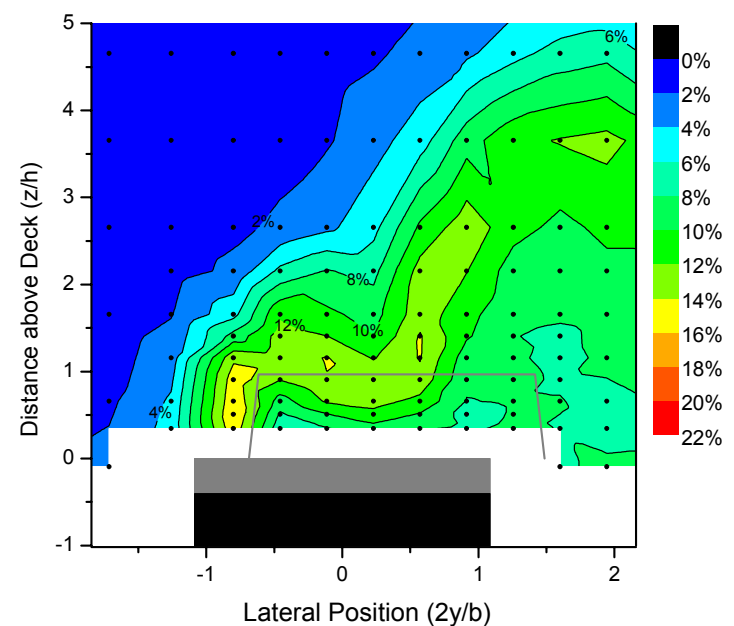

(a)

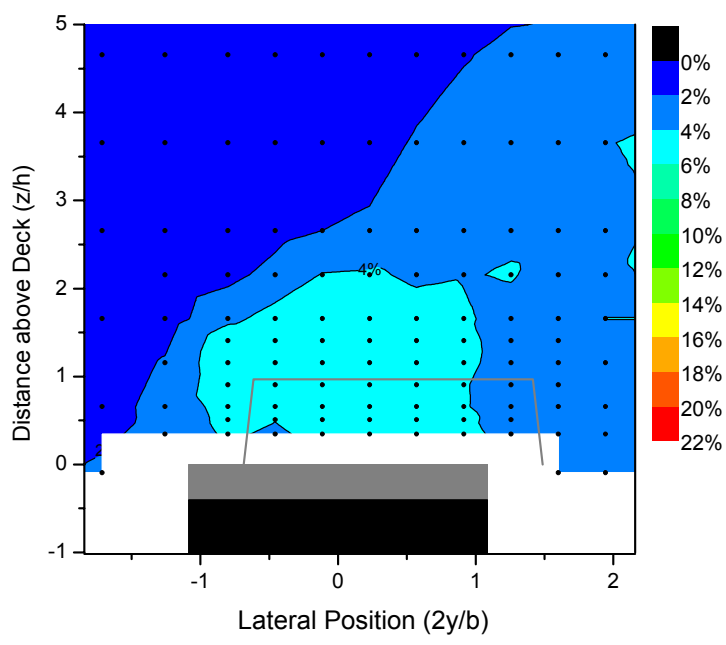

(b)

Figure 5.16. Baseline velocity standard deviation for the 20 degrees WOD runs: (a) the 0 - $125 \mathrm{~Hz}$ domain; (b) the $125-2000 \mathrm{~Hz}$ domain.

Figure 5.17 shows the frequency breakdown of the reticulated foam fence configuration at 20 degrees WOD. The peak in standard deviation downstream of the windward hangar corner consists of increased low frequency unsteadiness as well as high frequency content. The contribution of the high frequency unsteadiness, however, does not contribute to pilot workload. Also, the fence has "cleared out" a substantial region of low frequency unsteadiness behind the hangar. Figure 5.18 shows the contours for the configuration with porous surfaces and injection (run 18). This control technique does not reduce low frequency unsteadiness as much as the foam fence, but still shows improvements over the baseline. The majority of the high frequency content now forms from the edges of the hangar on the windward half of the ship. 


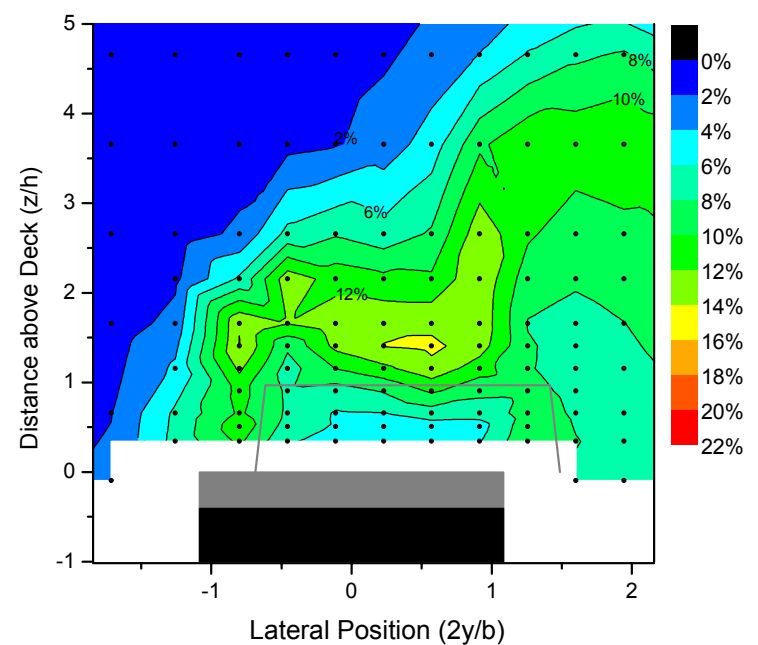

(a)

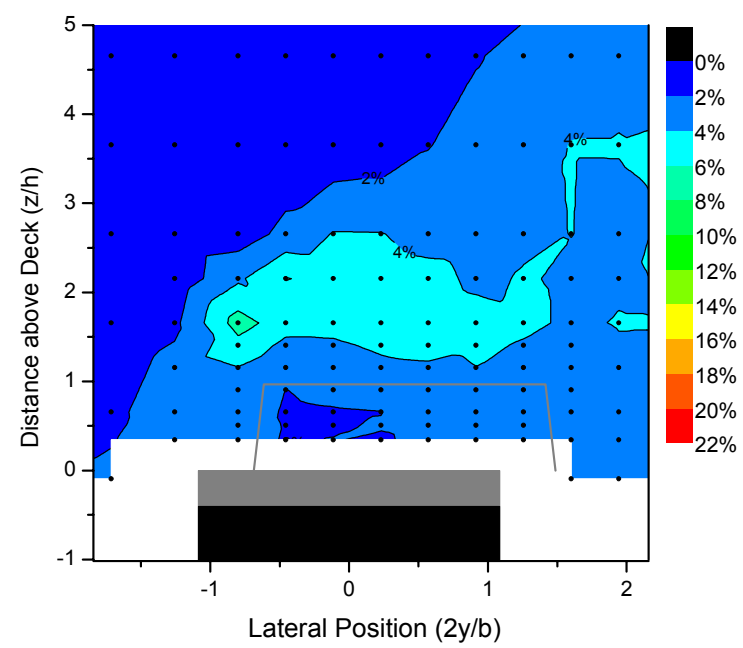

(b)

Figure 5.17. Velocity standard deviation at 20 degrees WOD for the porous flight deck with blowing from the hangar face for: a) the $0-125 \mathrm{~Hz}$ domain; b) the $125-2000 \mathrm{~Hz}$ domain.

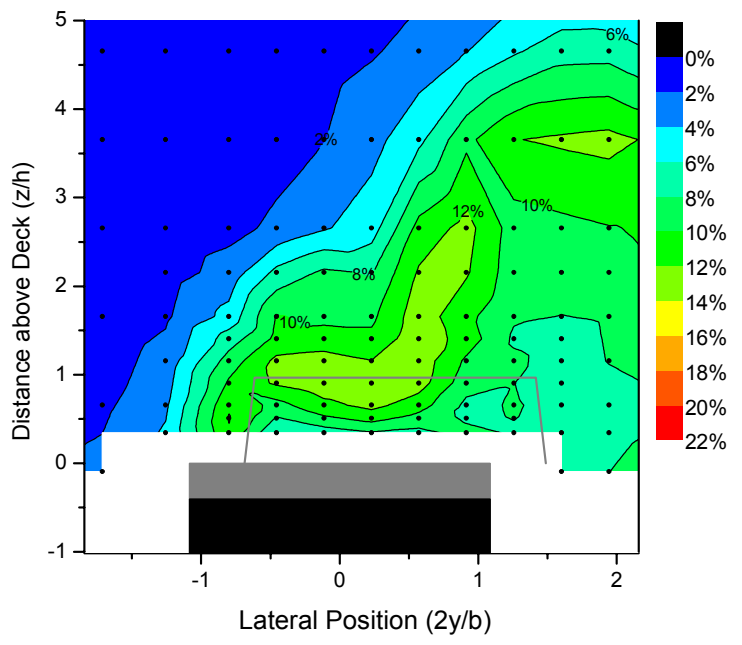

(a)

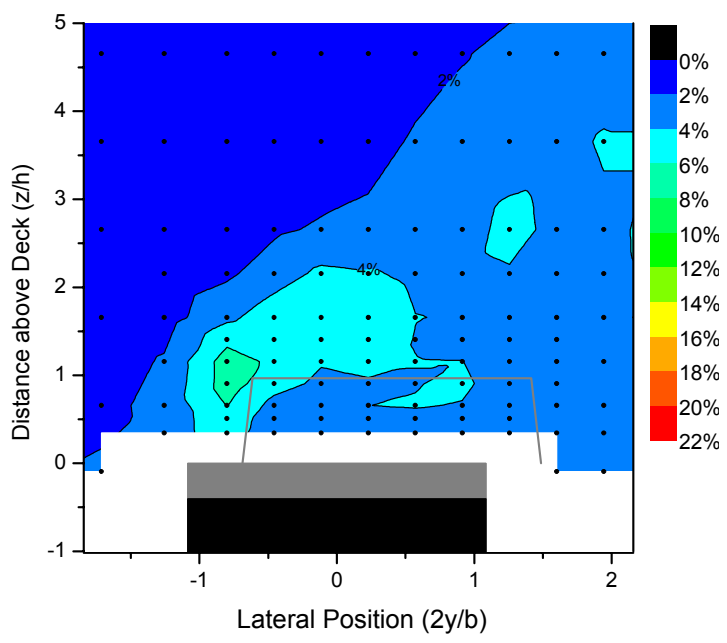

(b)

Figure 5.18. Velocity standard deviation at 20 degrees WOD for the reticulated foam fence configuration in: a) the 0 - $125 \mathrm{~Hz}$ domain; b) the $125-2000 \mathrm{~Hz}$ domain.

\subsection{Comparison to 0 degrees WOD}

With the exception of a few comments made earlier in this chapter, little has been said about the differences between 0 and 20 degrees WOD angles. Although Chapter 1 discussed the fundamental physical differences, a quantitative analysis is helpful. 
The following four figures show how these cases compare by including data from both WOD angles on a single plot. As seen in the figures, the 20 degree WOD cases are represented with solid lines and the 0 degree WOD cases with dashed lines. Only the foam fence configuration and the porous surface with injection configuration are included in the figures as these were the only two devices tested at both WOD angles.

Figure 5.19 shows the mean velocity at a horizontal cut of $z / h=0.66$. By analyzing the plot, one can visualize how the flow field changes as the WOD angle is increased. A similarity pattern can be noticed, and the two devices seem to consistently lower the mean velocity. The velocity standard deviation (Figure 5.20) shows the same trends with this horizontal cut of data. However, one interesting feature is observed. At 0 degrees WOD the foam fence decreases the unsteadiness across the flight deck, only to increase it just beyond the side of the ship. In the 20 degree WOD case, the fence does not cause this increase on either side of the ship. As a result, the disadvantages of the fence at 0 degree WOD do not occur at 20 degree WOD. 


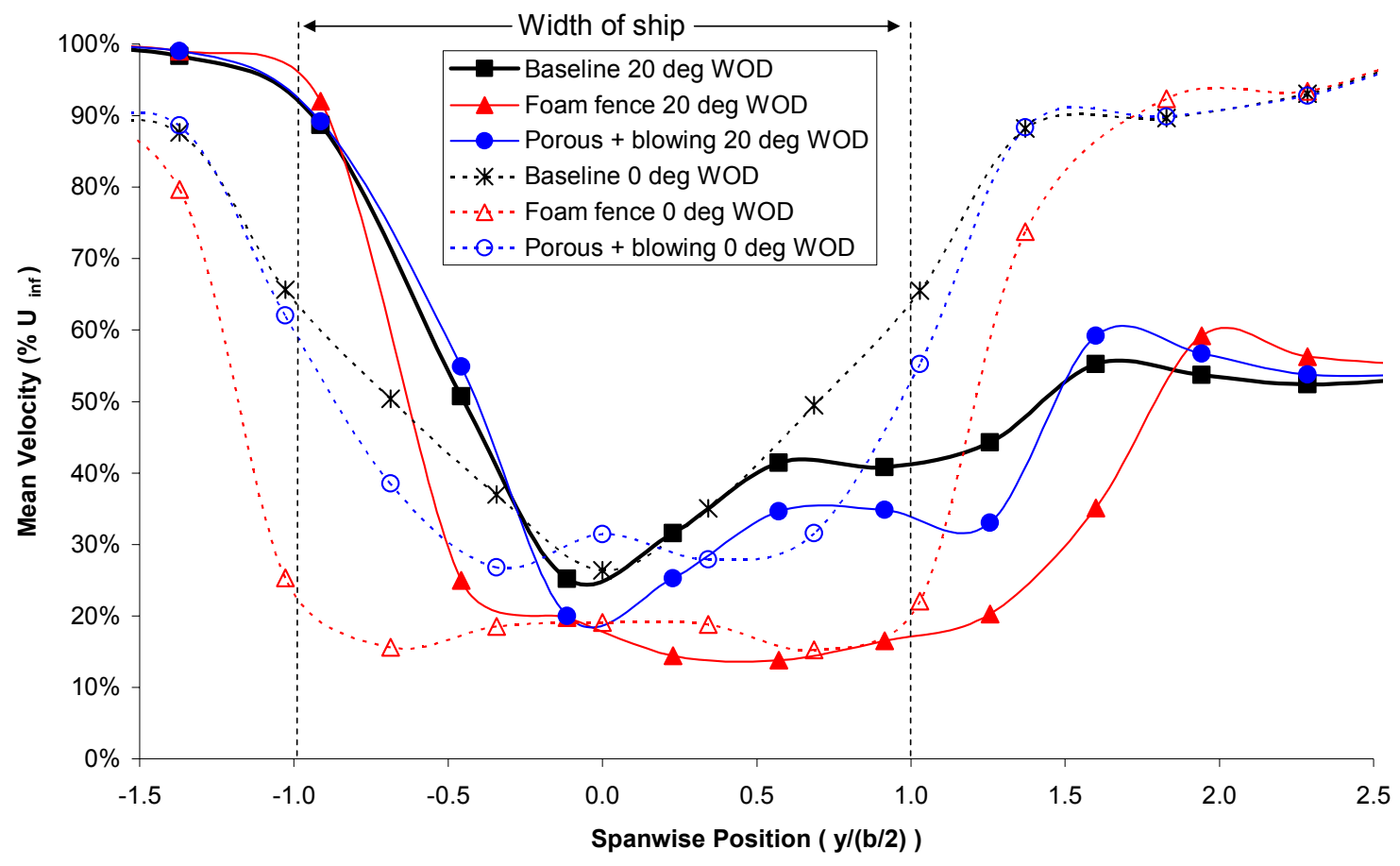

Figure 5.19. Comparison of mean velocity at 0 and 20 degrees WOD at $z / h=0.66$.

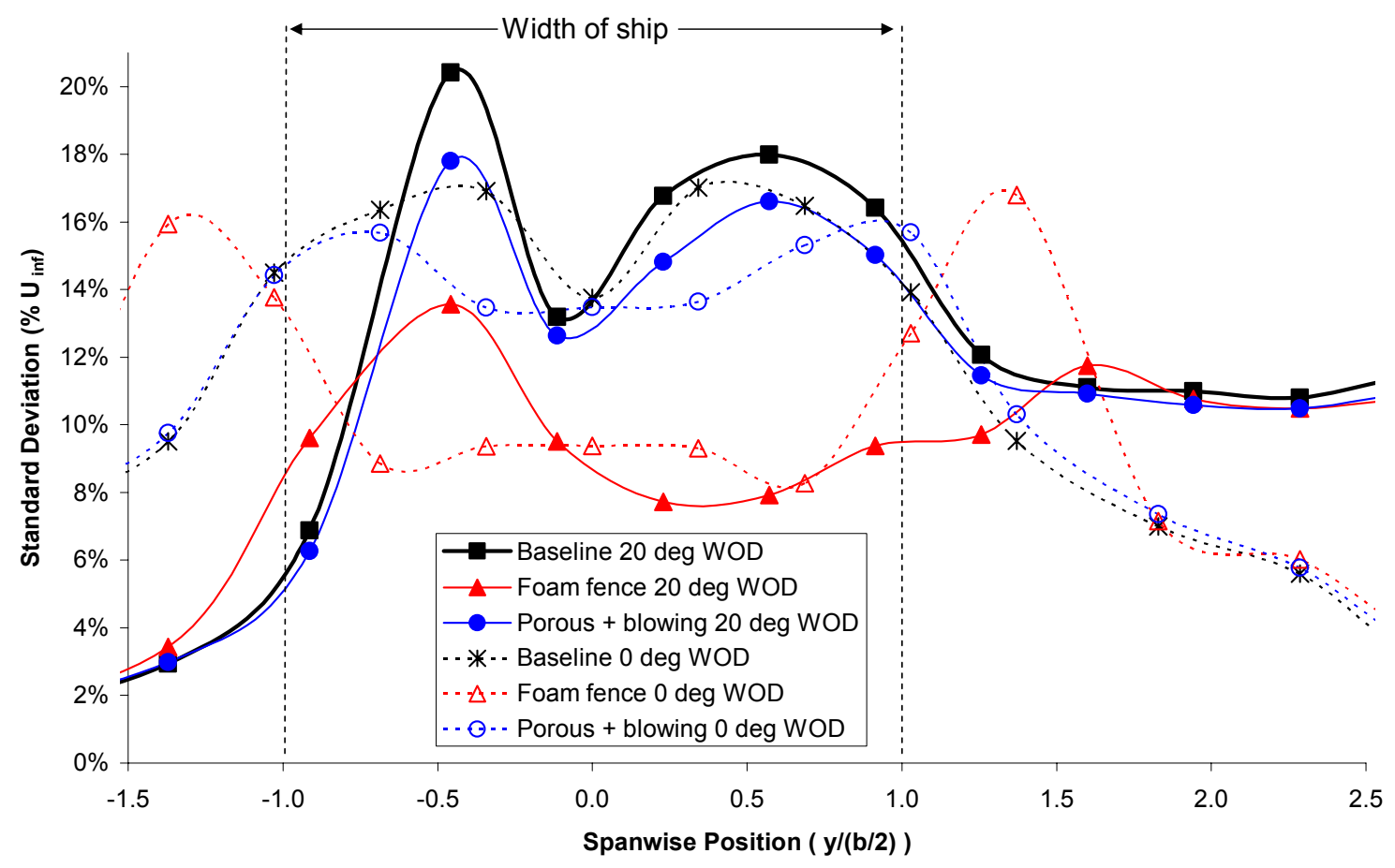

Figure 5.20. Comparison of standard deviation at 0 and 20 degrees WOD at $z / h=0.66$. 
A vertical cut through the data plane is also analyzed at the two different WOD angles. Due to the varying location of grid points between the cases, the 0 degree WOD data was taken at $2 y / b=0$, whereas the 20 degree WOD data was taken at $2 y / b=0.23$. Because vertical trends in the flow field are sought, this small difference is acceptable. Again, similar trends in the mean velocity are shown in Figure 5.21. The velocity standard deviation plot in Figure 5.22 shows greater variations between configurations. First, a large difference is noticed between the baselines of each WOD angle. The 20 degrees WOD case is significantly more unsteady than the 0 degrees WOD case. Although the porous surfaces and injection affect the two WOD cases similarly, the foam fence again shows interesting characteristics. For the 0 degrees WOD case, the fence reduces the unsteadiness behind the hangar, but causes an increase just a short distance above. This peak of maximum standard deviation increased from about $14 \%$ to $17 \%$ of the freestream velocity. However, when the WOD was changed to 20 degrees, the fence no longer causes an increase in maximum unsteadiness. Instead, the maximum actually decreased.

In summary, the comparison of the two WOD angles has shown that the configuration with porous surfaces and injection consistently improved the flow field regardless of WOD angle. However, the reticulated foam fence performed better. Although some disadvantages of using a fence configuration at 0 degrees WOD existed, they were eliminated in the 20 degrees WOD case. As a result, a reticulated foam fence is considered the best overall flow control device. 


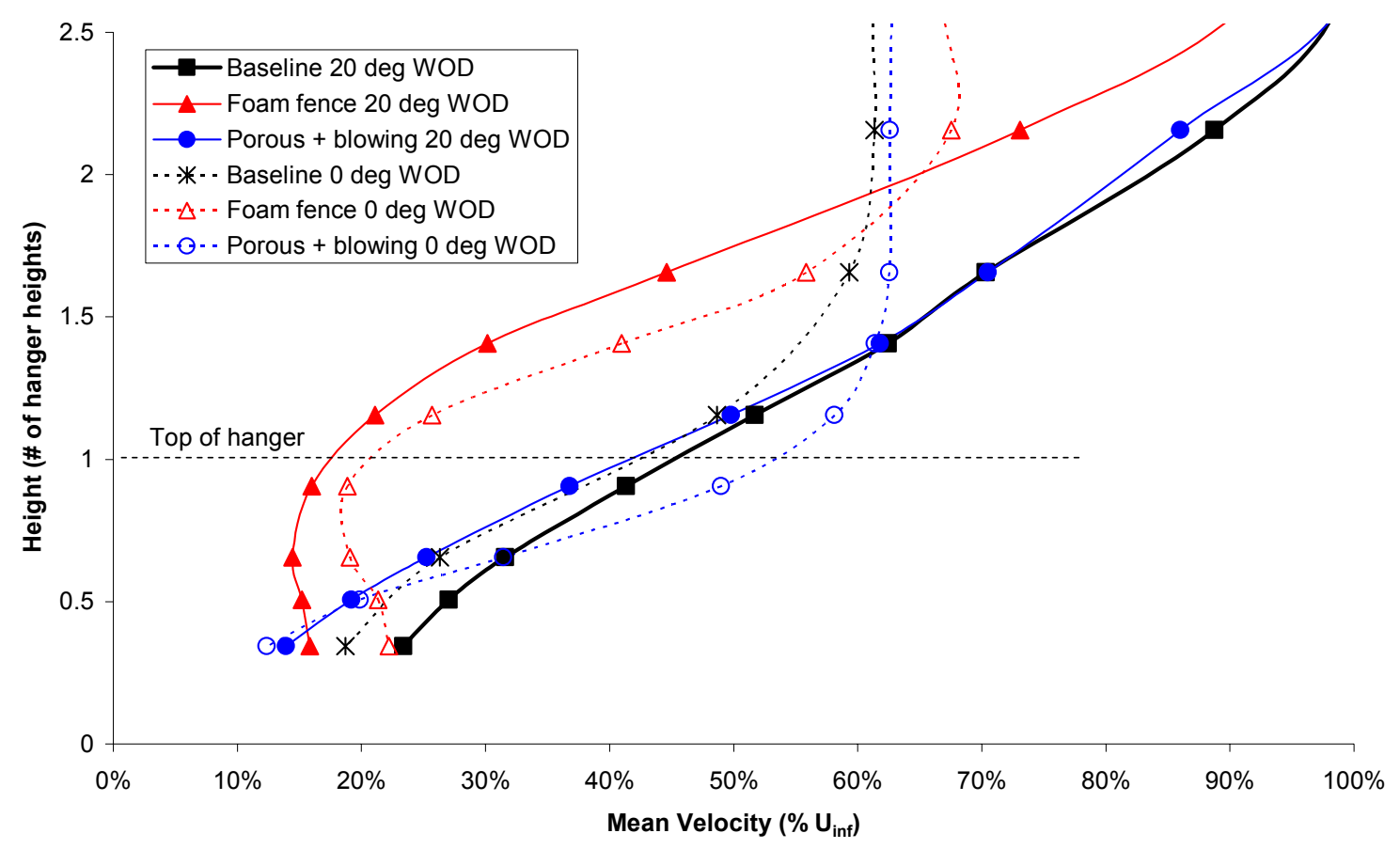

Figure 5.21. Comparison of mean velocity at 0 and 20 degrees WOD near the vertical centerline.

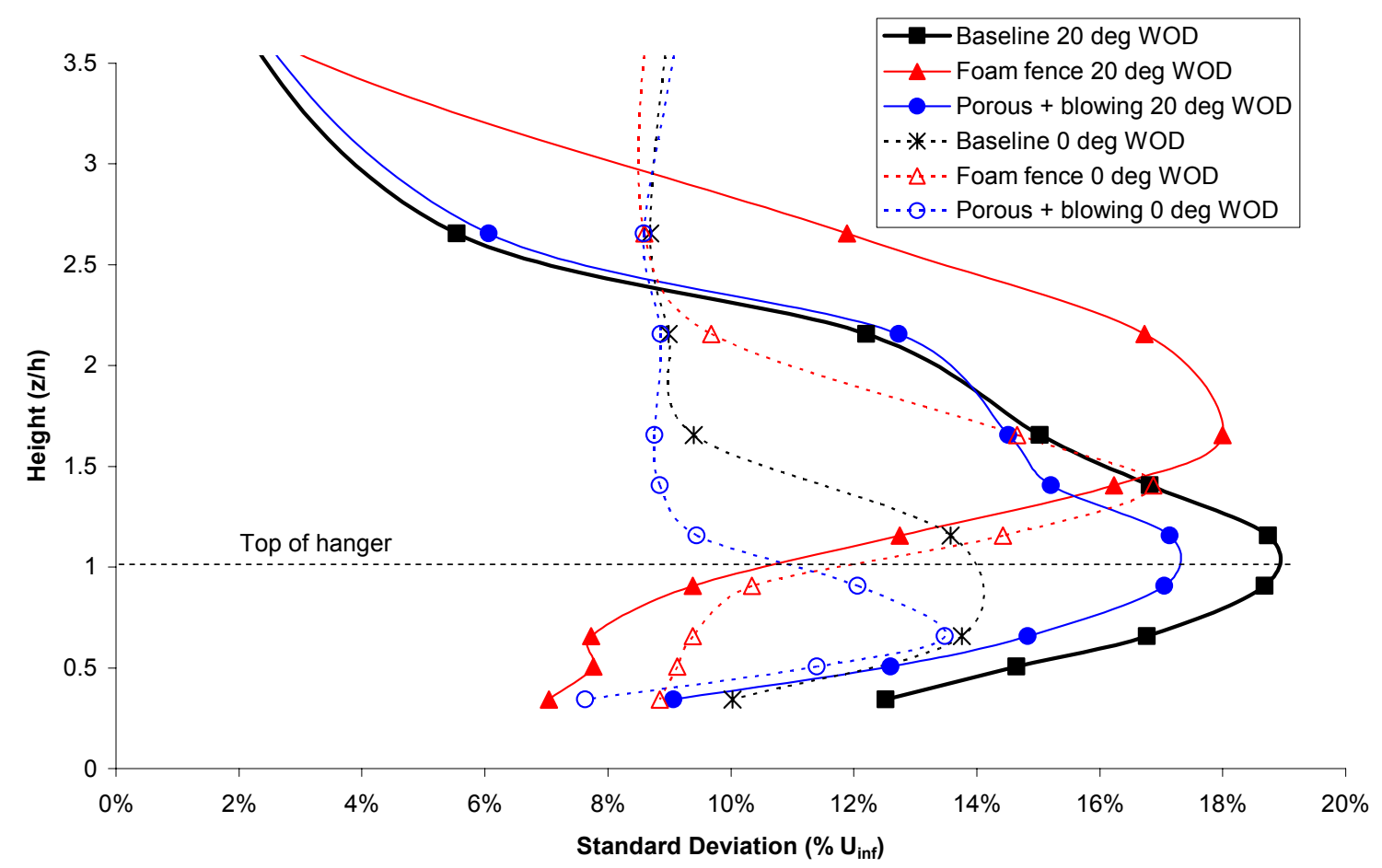

Figure 5.22. Comparison of standard deviation at 0 and 20 degrees WOD near the vertical centerline. 


\section{CONCLUSIONS AND RECOMMENDATIONS}

The flow over the rear landing deck of frigate-type ships is unsteady which makes it difficult for safe helicopter/ship operations. In this work, several different flow control techniques were wind tunnel tested at 0 degrees wind-over-deck (WOD). Data were collected in a vertical plane perpendicular to the freestream above the landing spot on the flight deck. The standard deviation of the velocity time history was chosen as the primary metric as it provides a measure of total unsteadiness in the flow. To compare the different flow control devices quantitatively, a metric was created which calculates the average unsteadiness in the region that the helicopter operates during approach and landing. The investigation also evaluated two flow control techniques at 20 degrees WOD.

The first several control techniques replaced the solid flight deck and hangar face with porous surfaces. These configurations were tested at 0 degrees WOD only. Although they were passive and did not change the ship's geometry, a small reduction $(2.3 \%$ to $4.1 \%)$ in unsteadiness was recorded in the landing region. Active configurations using mass injection through the porous surfaces were also tested. The best of these techniques involved channeling the injected air to exit through the hangar face. With an injection velocity of $2.5 \%$ of the freestream velocity, the unsteadiness in the landing region was reduced $6.6 \%$. This configuration was also tested at 20 degrees WOD, resulting in an $8.3 \%$ reduction over the baseline at this WOD angle.

The next series of tests involved evaluating several fence configurations placed around the perimeter of the hangar. Tested at 0 degrees WOD, these configurations cause 
reductions in unsteadiness downstream of the hangar face. However, these configurations cause a shear layer of increased unsteadiness just above hangar height, compared to the baseline. It is difficult to determine if this tradeoff is acceptable as the average unsteadiness in the landing region increases compared to the baseline for these configurations. The reticulated foam fence offers a compromise between these tradeoffs. This configuration caused the greatest reduction in unsteadiness downstream of the hangar and only a moderate increase near the top of the fence. At 20 degrees WOD the reticulated foam fence caused a $12.1 \%$ reduction in unsteadiness in the landing region, the highest of all the devices and WOD angles tested.

Several recommendations are made for future investigations:

Do not limit the investigation to 0 degrees wind-over-deck (WOD) cases. Because wind can come from any direction at sea, the WOD angle is rarely exactly 0 degrees, and the flow control techniques tested show greater improvements over the baselines at 20 degrees WOD.

$>$ Investigate fences attached to the sides of the hangar only. The unsteadiness caused by flow over the top fence and hangar may be decreased. The fences on the hangar sides may be responsible for the decreased unsteadiness downstream of the hangar face. It was also shown that fences located in areas of higher mean velocity were capable of breaking up flow structures into higher frequency turbulence as shown in Section 4.5.3. 
Optimize the use of reticulated foam fences. Various cross-sections and heights should be explored as this flow control technique has been shown to substantially reduce unsteadiness at 20 degrees WOD.

Consider fences that "push" the unsteady shear layer completely above the landing region. This requires a fence height of 1 to 2 times the height of the hangar. The average unsteadiness analysis in Section 4.4 showed that using larger fences may reduce the unsteadiness where improvements are needed the most.

Investigate configurations which have a higher injection velocity. Increasing this velocity beyond the $2.5 \%$ of freestream in this investigation would likely show much larger changes in the flow field. Using a baffling system which distributes the injection uniformly is also important.

A frequency analysis should be completed with focus placed on the unsteady fluctuations in the frequency domain which causes increased pilot workload. Low frequency content from 0.1 hertz to 2 hertz in full scale is the main contributor to workload. ${ }^{33}$ Integrating the PSD in this range provides a measure of unsteadiness in that important frequency domain. 


\section{REFERENCES}

${ }^{1}$ Healey, J. V., "The Prospects for Simulating the Helicopter/Ship Interface", Naval Engineers Journal, pp. 45-63, Mar. 1987.

${ }^{2}$ Rhoades, M. M. and Healey, J. V., "Flight Deck Aerodynamics of a Nonaviation Ship", Journal of Aircraft, Vol. 29, No. 4, pp. 619-626, 1992.

${ }^{3}$ Driver, David M. and Seegmiller, H. Lee, "Features of a Reattaching Turbulent Shear Layer in Divergent Channel Flow", AIAA Journal, Vol. 23, No. 2, pp. 163-171, 1985.

${ }^{4}$ Eaton, J. and Johnston, J., "A Review of Research on Subsonic Turbulent-Flow Reattachment”, AIAA Journal, Vol. 19, No. 9, pp. 1093-1100, 1980.

${ }^{5}$ Bhattacharjee, B. Scheelke, and T. R. Troutt, "Modification of Vortex Interactions in a Reattaching Separated Flow", AIAA Journal, Vol. 24, No. 4, pp. 623-629, 1986.

${ }^{6}$ Simpson, R. L., “Turbulent Boundary Layer Separation”, Annual Review of Fluid Mechanics, Vol. 21, pp. 205-234, 1989.

${ }^{7}$ Driver, D. M., Seegmiller, H. L., and Marvin, J. G., "Time-Dependent Behavior of a Reattaching Shear Layer”, AIAA Journal, Vol 25, No. 7, pp. 914-919, 1987.

${ }^{8}$ Castro, I. P., and Haque, A., "The Structure of a Turbulent Shear Layer Bounding a Separation Region”, Journal of Fluid Mechanics, Vol. 179, pp. 439-468, 1987.

${ }^{9}$ Ruderich, R., and Fernholz, H. H., “An Experimental Investigation of a Turbulent Shear Flow with Separation, Reverse Flow, and Reattachment", Journal of Fluid Mechanics, Vol. 163, pp. 283-322, 1986.

${ }^{10}$ Nguyen, T. M., Watkins, S., Saunders, J. W., "The Crosswind Dynamic Force on Three-dimensional Rectangular Cylinders in Turbulent Winds", $5^{\text {th }}$ International Colloquium on Bluff Body Aerodynamics and Applications, Ottawa, CA, July 2004.

${ }^{11}$ Rhoades, Mark M., "A Study of the Airwake Aerodynamics Over the Flight Deck of an AOR Model Ship", MS Thesis, Naval Postgraduate School, Monterey, CA, Sept. 1990.

12 Johns, Michael K., "Flow Visualization of the Airwake Around a Model of a DD-963 Class Destroyer in a Simulated Atmospheric Boundary Layer", MS Thesis, Naval Postgraduate School, Monterey, CA, Sept. 1988.

${ }^{13}$ Zan, S. J., "Surface Flow Topology for a Simple Frigate Shape", Canadian Aeronautics and Space Journal, Vol. 47, No.1, pp. 33-43, Mar. 2001. 
${ }^{14}$ Polsky, S. A., "CFD Prediction of Airwake Flowfields for Ships Experiencing Beam Winds", AIAA 2003-3657, AIAA Applied Aerodynamics Conference, Orlando, FL, Jun 2003.

${ }^{15}$ Polsky, S. A., "A Computational Study of Unsteady Ship Airwake", AIAA 20021022, AIAA Aerospace Sciences Meeting, Reno, NV, Jan. 2002.

${ }^{16}$ Polsky, Susan A. and Bruner, Christopher W. S., "Time-Accurate Computational Simulations of an LHA Ship Airwake", AIAA 2000-4126, AIAA Applied Aerodynamics Conference, Denver CO, Aug. 2000.

${ }^{17}$ Sharma, Anupam and Long, Lyle N., "Airwake Simulations on an LPD 17 Ship", AIAA 2001-2589, AIAA Computational Fluid Dynamics Conference, Anaheim, CA, Jun. 2001.

${ }^{18}$ Guillot, M. J. and Walker, M. A., "Unsteady Analysis of the Airwake Over the LPD17”, AIAA 2000-4125, AIAA Applied Aerodynamics Conference, Denver CO, Aug. 2000.

${ }^{19}$ Bunnell, John W., "An Integrated Time-Varying Airwake in a UH-60 Black Hawk Shipboard Landing Simulation", AIAA 2001-4065, AIAA Modeling and Simulation Technologies Conference and Exhibit, Montreal, Canada, Aug. 2001.

${ }^{20}$ Anderson, Gustav A., "Mapping the Airwake of a Model DD-963 Along Specific Helicopter Flight Paths", MS Thesis, Naval Postgraduate School, Monterey, CA, Dec. 1989.

${ }^{21}$ Walker, Monica A., Kimmel, Kevin R., "Wind Tunnel Investigation of the Unstaedy Air Wake Along the Flight Path Behind CVN-73 and CVN-76 Aircraft Carrier Configurations", Naval Surface Warfare Center CRDKNSWC/HD-1422-04, Apr. 1996.

${ }^{22}$ Lee, Richard G. and Zan, Steven J., "Unsteady Aerodynamic Loading on a Helicopter Fuselage in a Ship Airwake", Journal of the American Helicopter Society, pp. 149159, Apr. 2004.

${ }^{23}$ Lee, Richard G. and Zan, Steven J., "Wind Tunnel Testing of a Helicopter Fuselage and Rotor in a Ship Airwake", $29^{\text {th }}$ European Rotorcraft Forum, Friedrichshafen, Germany, Sept. 2003.

${ }^{24}$ Zan, S. J., "Experimental Determination of Rotor Thrust in a Ship Airwake", Journal of the American Helicopter Society, pp.100-108, Apr. 2002.

${ }^{25}$ Biskaduros, John L., "Flow Visualization of the Airwake of an Oscillating Generic Ship Model”, MS Thesis, Naval Postgraduate School, Monterey, CA, Dec. 1987. 
${ }^{26}$ Woolman, Scott G., "Control of Flow Over a Backward Facing Step”, MS Thesis, Naval Postgraduate School, Monterey, CA, Sept. 1990.

${ }^{27}$ Narveson, Marshall L., "Flow Modification Over a Backward Facing Step", MS Thesis, Naval Postgraduate School, Monterey, CA, Sept. 1990.

${ }^{28}$ Spazzini, P. G., Di Cicca, G. M., Iuso, G., King, R., Onorato, M., “Flow Control Downstream of a Backward Facing Step: A DPIV Study", Proceedings of International Congress of Aerospace Sciences, ICAS 2002-3.9.4. Sept. 2002.

${ }^{29}$ Heenan, A. F. and Morrison, J. F., "Passive Control of Pressure Fluctuations Generated by Separated Flow", AIAA Journal, Vol. 36, No. 6, pp. 1014-1022, 1998.

${ }^{30}$ Nangia, R. K. and Lumsden, R. B., "Novel Vortex Flow Devices - Columnar Vortex Generators Studies for Airwakes", AIAA 2004-2348, AIAA Fluid Dynamics Conference, Portland, OR, Jul. 2004.

${ }^{31}$ Zan, S. J., teleconference, 1 Sept. 2004.

${ }^{32}$ Yaragal, S. C., Ram, H. S. Govinda, Murthy, K. Keshava, “An experimental investigation of flow fields downstream of solid and porous fences", Journal of Wind Engineering and Industrial Aerodynamics, Vol. 66, No. 2, pp. 127-140, 1997.

${ }^{33}$ McRuer, Duane T., "Interdisciplinary interactions and dynamic systems integration", International Journal of Control, Vol. 59, No. 1, pp. 3-12, 1994.

${ }^{34}$ Stewart, V.R., "Naval Air Warfare Center, Warminster, PA Aerodynamic Test Facility Operation Instructions", KSA Technology Report, Columbus, OH, Aug. 1996.

${ }^{35}$ Rae, W.H., Pope, A., Low-Speed Wind Tunnel Testing, $2^{\text {nd }}$ Edition, Wiley, New York, 1984.

${ }^{36}$ Bruun, H. H. Hot-Wire Anemometry. Oxford University Press, New York, pp.127-31, 1995.

${ }^{37}$ Baker, Andrew, "Re: Helo approach to DDG”, email to Daniel Shafer, 17 Sept. 2004.

${ }^{38}$ Smith, Steven W. The Scientist's and Engineer's Guide to Digital Signal Processing. California Technical Publishing, 1997.

${ }^{39}$ Schlichting, Hermann. Boundary-Layer Theory. McGraw-Hill Book Company, $7^{\text {th }}$ ed, p. 32, 1979.

${ }^{40}$ Pope, Stephen B. Turbulent Flows. Cambridge University Press, 2003. 


\section{APPENDIX A}

\section{CALCULATION OF STANDARD DEVIATION}

The IFA 100 constant temperature hotwire manual ${ }^{*}$ defines the standard deviation of the velocity as

$$
\sigma=\sqrt{\mu_{2}}
$$

where $\mu_{2}$ is the normal stress or the second moment about the mean, defined as

$$
\mu_{2}=M_{2}-M_{1}^{2}
$$

where

$$
\begin{aligned}
& M_{2}=\frac{1}{n} \sum_{i=1}^{n} x_{i}^{2} \\
& M_{1}=\frac{1}{n} \sum_{i=1}^{n} x_{i}=\bar{x} \Rightarrow \text { mean }
\end{aligned}
$$

Combining equations, the IFA 100 calculates standard deviation using

$$
\sigma=\sqrt{\frac{\sum x_{i}^{2}-n \bar{x}^{2}}{n}}
$$

However, a statistics reference ${ }^{\dagger}$ defines standard deviation as

$$
\sigma=\sqrt{\frac{\sum\left(x_{i}-\bar{x}\right)^{2}}{n}}
$$

The following proof shows that the two expressions are equal. Expanding equation (6),

$$
\sigma=\sqrt{\frac{\sum\left(x_{i}^{2}-2 x_{i} \bar{x}+\bar{x}^{2}\right)}{n}}
$$

\footnotetext{
* Thermal Systems, Inc., Model IFA 300 Constant Temperature Anemometer System user's manual.

$\dagger$ Volk, William. Applied Statistics for Engineers. New York: McGraw-Hill Book Company, Inc., 1958.
} 


$$
\sigma=\sqrt{\frac{\sum x_{i}^{2}-2 \bar{x} \sum x_{i}+\sum \bar{x}^{2}}{n}}
$$

Noting that $\sum x_{i}=n \bar{x}$ and $\sum \bar{x}^{2}=n \bar{x}^{2}$,

$$
\begin{aligned}
& \sigma=\sqrt{\frac{\sum x_{i}^{2}-2 \bar{x} n \bar{x}+n \bar{x}^{2}}{n}} \\
& \sigma=\sqrt{\frac{\sum x_{i}^{2}-n \bar{x}^{2}}{n}}
\end{aligned}
$$

Equation (10) is the same expression as equation (5), proving the consistency of standard deviation expressions. This expression is also the root-mean-square (RMS) of the fluctuations of a signal. Because $\bar{x}$ is zero for fluctuations about the mean, standard deviation and RMS are equal. 


\section{APPENDIX B \\ DERIVATION OF RESULTANT MEAN \\ VELOCITY FOR SINGLE COMPONENT HOT WIRE}

A single component hot wire measures the resultant velocity in the freestream and vertical directions. Therefore,

$$
q=\sqrt{u^{2}+w^{2}}
$$

Decomposing $u$ and $w$ into mean and fluctuating components,

$$
\begin{gathered}
q=\sqrt{\bar{u}^{2}+2 \bar{u} u^{\prime}+u^{\prime 2}+\bar{w}^{2}+2 \bar{w} w^{\prime}+w^{\prime 2}} \\
q=\sqrt{\left(\bar{u}^{2}+\bar{w}^{2}\right)\left[1+\frac{2 \bar{u} u^{\prime}}{\bar{u}^{2}+\bar{w}^{2}}+\frac{2 \bar{w} w^{\prime}}{\bar{u}^{2}+\bar{w}^{2}}+\frac{u^{\prime 2}}{\bar{u}^{2}+\bar{w}^{2}}+\frac{w^{\prime 2}}{\bar{u}^{2}+\bar{w}^{2}}\right]}
\end{gathered}
$$

Neglecting the terms that are second order small,

$$
q=\sqrt{\left(\bar{u}^{2}+\bar{w}^{2}\right)\left[1+\frac{2 \bar{u} u^{\prime}}{\bar{u}^{2}+\bar{w}^{2}}+\frac{2 \bar{w} w^{\prime}}{\bar{u}^{2}+\bar{w}^{2}}+O\left(u^{\prime 2}, w^{\prime 2}\right)\right]}
$$

Rewriting the expression using the binomial approximation,

$$
\begin{gathered}
q=\sqrt{\left(\bar{u}^{2}+\bar{w}^{2}\right)}\left[1+\frac{1}{2}\left(\frac{2 \bar{u} u^{\prime}}{\bar{u}^{2}+\bar{w}^{2}}+\frac{2 \bar{w} w^{\prime}}{\bar{u}^{2}+\bar{w}^{2}}\right)+O\left(u^{\prime 2}, w^{\prime 2}\right)\right] \\
q=\sqrt{\left(\bar{u}^{2}+\bar{w}^{2}\right)}+\frac{\bar{u} u^{\prime}}{\sqrt{\bar{u}^{2}+\bar{w}^{2}}}+\frac{\bar{w} w^{\prime}}{\sqrt{\bar{u}^{2}+\bar{w}^{2}}}+O\left(u^{\prime 2}, w^{\prime 2}\right)
\end{gathered}
$$

From this expression, it is observed that for small values of $u^{\prime}$ and $w^{\prime}$ (when the second order terms can be neglected), time averaging causes the first order unsteady terms to drop out. This results in the following expression for the mean resultant velocity: 


$$
\bar{q}=\sqrt{\left(\bar{u}^{2}+\bar{w}^{2}\right)}
$$

The resultant fluctuation, $q^{\prime}$, is the velocity fluctuation in the direction of $\bar{q}$, the mean velocity vector. Note that if the fluctuations are not small, then the second order terms cannot be neglected and the expression for the resultant mean velocity is not trivial. 


\section{APPENDIX C \\ CONTOUR INTERPOLATION TECHNIQUE}

The experimental data grid shown in Figure 3.6 shows that data points were concentrated in the region of the backward facing step. In practice, to create contour plots of data in a measurement area, the data must be in an equally spaced rectangular grid. This is necessary so that the plotting software does not utilize its own "black box" interpolation scheme. Linear interpolation was used to create the uniformly spaced grid although more complex schemes are available.

Linear interpolation in a two-dimensional data plane requires the use of Delaunay triangulation. This procedure creates a series of triangles by connecting the grid points. If the vertices of each triangle are used to define a circle, the interior of this circle will not contain any other grid points. As a result, each point is connected with its "natural neighbors". After these triangles are formed, the value of any point on the interior can be calculated by a method outlined in Watson's text

The following MATLAB script calculated the uniformly spaced rectangular grid required for contour plotting.

function datainterp $=$ hwinterp(method,runnum,type)

$\%$ This function calls a data file which was created using hwdata.m.

$\%$ Example:

$\%$ For a linear interpolation of Run 1 standard deviation data, type:

$\%$ hwinterp('linear',1,'stdev');

\footnotetext{
*Watson, David F., Contouring: A Guide to the Analysis and Display of Spatial Data New York: Pergamon Press, 1992.
} 


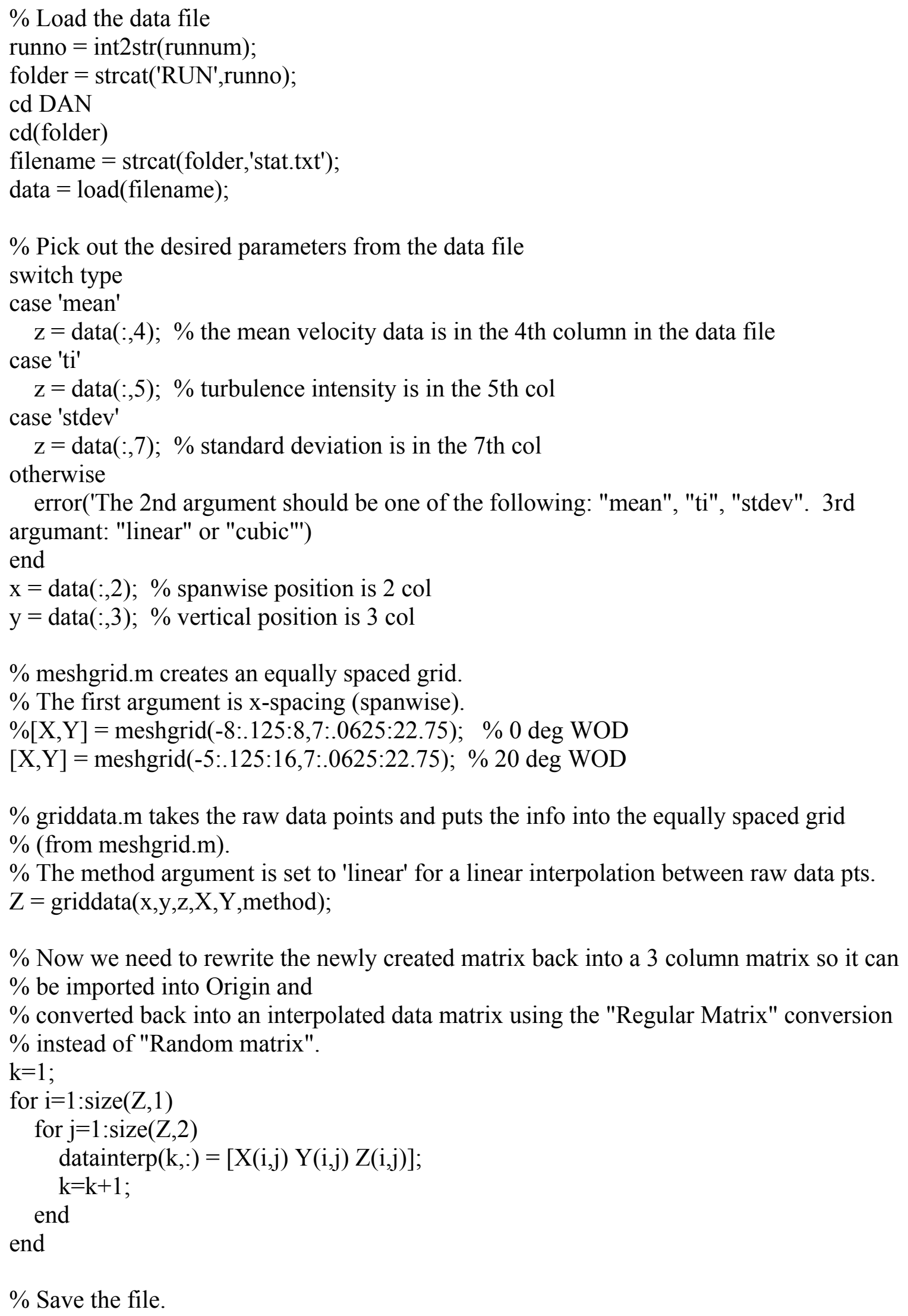

$\%$ Now we need to rewrite the newly created matrix back into a 3 column matrix so it can $\%$ be imported into Origin and

$\%$ converted back into an interpolated data matrix using the "Regular Matrix" conversion $\%$ instead of "Random matrix".

$\mathrm{k}=1$;

for $\mathrm{i}=1: \operatorname{size}(\mathrm{Z}, 1)$

for $\mathrm{j}=1: \operatorname{size}(Z, 2)$ datainterp $(k,:)=[X(i, j) Y(i, j) Z(i, j)]$; $\mathrm{k}=\mathrm{k}+1$;

$\%$ Save the file. 
filename2 = strcat(method,folder,type,'.txt');

cd ..

cd interpolated

save(filename2,'datainterp','-ascii')

cd..

cd.. 


\section{APPENDIX D}

\section{POWER SPECTRAL DENSITY CALCULATION}

The following MATLAB script was used to generate the power spectral density plots as presented in Sections 4.5 and 5.5.

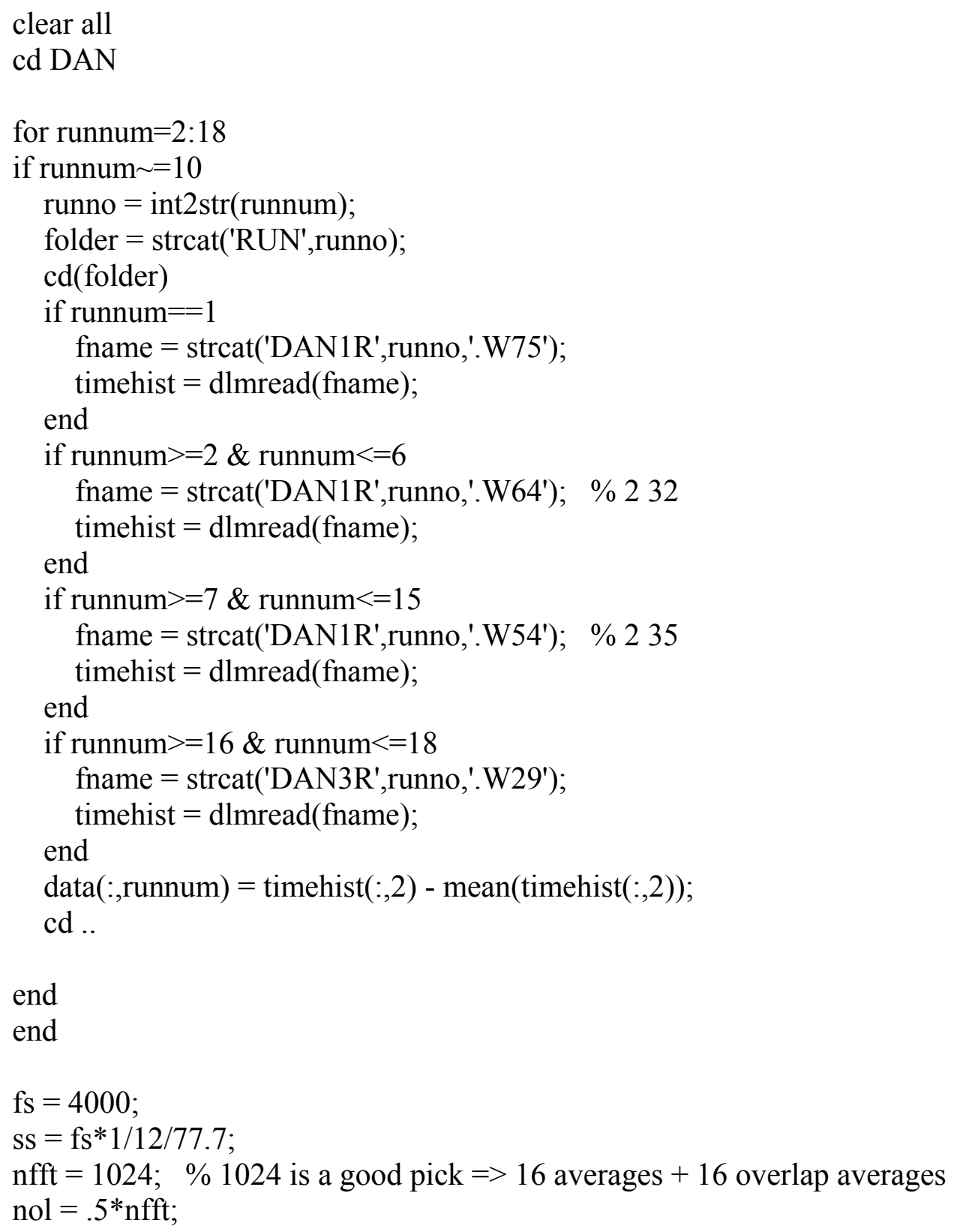




$$
\begin{array}{r}
\mathrm{B}=\text { firl }(40,3) ; \quad \% \text { use } .3 \text { for low freq when } \mathrm{nfft}=1024 \\
\% \text { use } .3 / 4 \text { for full spec when } \mathrm{nfft}=1024
\end{array}
$$

$\mathrm{A}=$ zeros(length $(\mathrm{B}), 1)$

$\mathrm{A}(1)=1$

colors = ['k-';'b-';'r-';'g-';'m-';'y-';'c-';'k:';'b:';'r',';g:';'m:';'y:';'c:';'--';'-'.];

runvec $=\left[\begin{array}{llllll}2 & 4 & 8 & 11 & 12 & 15\end{array}\right] ; \%$ choose runs to analyze

$\%$ pxx cols correspond to the run \#'s in the "runvec" variable

for $\mathrm{i}=1$ :length(runvec)

\%pwelch(data(:,runnum),nfft,nol,nfft,fs);

$[\operatorname{pxx}(:, \mathrm{i}), \mathrm{f}]=$ pwelch(data(:,runvec(i)),nfft,nol,nfft,fs);

$\operatorname{pxxf}(:, \mathrm{i})=$ filtfilt(B,A,pxx(:,i));

$\%[\mathrm{pxx}, \mathrm{f}]=\operatorname{psd}(\mathrm{data}, \mathrm{nfft}, \mathrm{fs}, \mathrm{nfft}, \mathrm{nol})$;

$\operatorname{sum}=0$

sumlf $=0$;

for $\mathrm{j}=1$ :length(f)- 1

if $\mathrm{j}<=($ length(f)-1) $/ 16-1 \% 0-125 \mathrm{~Hz}$ when divided by 16 ; sum $1=(f(j+1)-f(j)) *(p x x(j, i)+p x x(j+1, i)) / 2$; sumlf $=$ sum $1+$ sumlf;

end

$\operatorname{sum} 2=(f(j+1)-f(j)) *(p x x(j, i)+p x x(j+1, i)) / 2$;

sum $=\operatorname{sum} 2+$ sum ;

end

sum

ratio $=$ sumlf $/$ sum $* 100$

runno $=$ int $2 \operatorname{str}(\operatorname{runvec}(\mathrm{i}))$;

vname $=$ strcat('Ratio for Run ', runno, ' = ');

disp(vname)

disp(ratio)

figure(1)

hold on

$\operatorname{plot}(\mathrm{f}, 10 * \log 10(\operatorname{pxx}(:, \mathrm{i})), \operatorname{colors}(\mathrm{i}, \mathrm{:}))$

$\% \operatorname{axis}\left(\left[\begin{array}{llll}0 & 2000 & -40 & 0\end{array}\right]\right)$

grid on

if runvec(i) $>9$

legendtext(i,:) = ['Run' ' ' runno];

else

legendtext(i,:) = ['Run' ' ' runno];

end

legend(legendtext)

xlabel('Frequency $\left.(\mathrm{Hz})^{\prime}\right)$

\%xlabel('Strouhal Number (S)')

ylabel('Power Spectral Density (dB/Hz)') 
\%ylabel('Power Spectral Density (dB/S)')

set(gcf, 'color', 'white');

figure(2)

hold on

$\operatorname{plot}(\mathrm{f}, 10 * \log 10(\operatorname{pxxf}(:, \mathrm{i})), \operatorname{colors}(\mathrm{i},:))$

$\%$ axis([0 $2000-400])$

grid on

legend(legendtext)

xlabel('Frequency $\left.(\mathrm{Hz})^{\prime}\right)$

\%xlabel('Strouhal Number (S)')

ylabel('Power Spectral Density (dB/Hz)')

\%ylabel('Power Spectral Density $\left.(\mathrm{dB} / \mathrm{S})^{\prime}\right)$

set(gcf, 'color', 'white');

end

cd.. 


\section{VITA}

Born in Morgantown, WV on February 28, 1981, Dan Shafer moved with his father, mother, and sister to Oakland, MD in 1984. Dan first became fascinated with airplanes when his mother put space shuttle wallpaper in his room at a very young age. His babysitter, Kim, was the first one to put the words "aerospace engineer" in to his mouth, as she was studying in the field at the time. Throughout his childhood Dan spent his summers helping at the family pharmacy and enjoying the recreational haven Deep Creek Lake. His family has owned a small cottage on the lake since the 1950s. Deep Creek has given him countless memories from when he was a small boy to the present. In 1999, Dan graduated from Southern Garrett High School and was named valedictorian of his class. In the fall, he attended the University of Maryland - College Park, majoring in aerospace engineering. After only one semester of college, he enrolled in a pilot ground school course, and eventually became a private pilot. While at Maryland, Dan played trumpet at football and basketball games, was president of the Maryland Ski Team, and ran two marathons while maintaining above a 3.9 GPA. During this period, his fascination with aircraft continued to grow, so he decided to immediately pursue a Master of Science. Relocating to Blacksburg, VA gave Dan a chance to escape from the city/suburban lifestyle of College Park. Upon completion of his MS, he will begin his career as a civil service flight test engineer at Naval Air Station - Patuxent River, MD. 